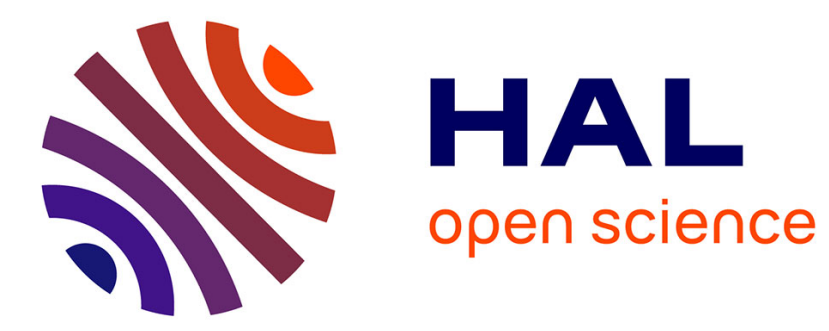

\title{
A stochastic target approach for P\&L matching problems
}

Bruno Bouchard, Vu Than Nam

\section{To cite this version:}

Bruno Bouchard, Vu Than Nam. A stochastic target approach for P\&L matching problems. Mathematics of Operations Research, 2012, 37 (3), pp.526-558. 10.1287/moor.1120.0549 . hal-00608649

\section{HAL Id: hal-00608649 \\ https://hal.science/hal-00608649}

Submitted on 13 Jul 2011

HAL is a multi-disciplinary open access archive for the deposit and dissemination of scientific research documents, whether they are published or not. The documents may come from teaching and research institutions in France or abroad, or from public or private research centers.
L'archive ouverte pluridisciplinaire HAL, est destinée au dépôt et à la diffusion de documents scientifiques de niveau recherche, publiés ou non, émanant des établissements d'enseignement et de recherche français ou étrangers, des laboratoires publics ou privés. 


\title{
A stochastic target approach for P\&L matching problems
}

\author{
Bruno Bouchard \\ CEREMADE, Université Paris Dauphine \\ and CREST-ENSAE \\ bouchard@ceremade.dauphine.fr
}

\author{
Vu Thanh Nam \\ CEREMADE, Université Paris Dauphine \\ thanh@ceremade.dauphine.fr
}

July 12, 2011

\begin{abstract}
Within a Brownian diffusion Markovian framework, we provide a direct PDE characterization of the minimal initial endowment required so that the terminal wealth of a financial agent (possibly diminished by the payoff of a random claim) can match a set of constraints in probability. Such constraints should be interpreted as a rough description of a targeted profit and loss (P\&L) distribution. This allows to give a price to options under a P\&L constraint, or to provide a description of the discrete $\mathrm{P} \& \mathrm{~L}$ profiles that can be achieved given an initial capital. This approach provides an alternative to the standard utility indifference (or marginal) pricing rules which is better adapted to market practices. From the mathematical point of view, this is an extension of the stochastic target problem under controlled loss, studied in Bouchard, Elie and Touzi (2009), to the case of multiple constraints. Although the associated Hamilton-Jacobi-Bellman operator is fully discontinuous, and the terminal condition is irregular, we are able to construct a numerical scheme that converges at any continuity points of the pricing function.
\end{abstract}

Key words: Stochastic target problem, Discontinuous viscosity solutions, Quantile Hedging.

AMS 1991 subject classifications: Primary 49L25, 60J60; secondary 49L20, 35K55.

\section{Introduction}

Option pricing (in incomplete financial markets or markets with frictions) and optimal management decisions have to be based on some risk criterion or, more generally, on some choice of preferences. In the academic literature, one usually models the attitude of the financial agents toward risk in terms of an utility or loss function. However, practitioners have in general no idea of "their utility function". Even the choice of a loss function is somehow problematic. On the other hand, they have a rough idea on the type of P\&L they can afford, and indeed have as a target. This is the case for traders, for hedge-fund managers,...

The aim of this paper is to provide a direct PDE characterization of the minimal initial endowment required so that the terminal wealth of a financial agent (possibly diminished by the payoff of a random claim) can match a set of constraints in probability. In practice, this set of constraints has to be viewed as a rough description of a targeted $\mathrm{P} \& \mathrm{~L}$ distribution. 
To be more precise, let us consider the problem of a trader who would like to hedge a European claim of the form $g\left(X_{t, x}(T)\right)$, where $X_{t, x}$ models the evolution of some risky assets, assuming that their value is $x$ at time $t$. The aim of the trader is to find an initial endowment $y$ and a hedging strategy $\nu$ such that the terminal value of his hedging portfolio $Y_{t, x, y}^{\nu}(T)$ diminished by the liquidation value of the claim $g\left(X_{t, x}(T)\right)$ matches an a-priori distribution of the form

$$
\mathbb{P}\left[Y_{t, x, y}^{\nu}(T)-g\left(X_{t, x}(T)\right) \geq-\gamma^{i}\right] \geq p^{i}, \quad i \leq \kappa,
$$

where $\gamma^{\kappa} \geq \cdots \geq \gamma^{2} \geq \gamma^{1} \geq 0$, for some $\kappa \geq 1$. The minimal initial endowment required to achieve the above constraints is given by:

$$
v(t, x, p):=\inf \left\{y: \exists \nu \text { s.t. } Y_{t, x, y}^{\nu}(T) \geq \ell \text { and } \mathbb{P}\left[Y_{t, x, y}^{\nu}(T)-g\left(X_{t, x}(T)\right) \geq-\gamma^{i}\right] \geq p^{i} \forall i \leq \kappa\right\},
$$

where we used the notation $p:=\left(p^{1}, \ldots, p^{\kappa}\right)$ and $\ell \in \mathbb{R}$ is a given lower bound that is imposed in order to avoid that the wealth goes too negative, even if it is with small probability.

In the case $\kappa=1$, such a problem is referred to as the "quantile hedging problem". It has been widely studied by Föllmer and Leukert [11] who provided an explicit description of the optimal terminal wealth $Y_{t, x, y}^{\nu}(T)$ in the case where the underlying financial market is complete. This result is derived from a clever use of the Neyman-Pearson Lemma in mathematical statistics and applies to non-Markovian frameworks. A direct approach, based on the notion of stochastic target problems, has then been proposed by Bouchard, Elie and Touzi [6]. It allows to provide a PDE characterization of the pricing function $v$, even in incomplete markets or in cases where the stock price process $X_{t, x}$ can be influenced by the trading strategy $\nu$, see e.g. [5]. The problem (1.1) is a generalization of this work to the case of multiple constraints in probability.

As in Bouchard, Elie and Touzi [6], the first step consists in rewriting the stochastic target problem with multiple constraints in probability (1.1) as a stochastic target problem in the $\mathbb{P}$-a.s. sense. This is achieved by introducing a suitable family of $d$-dimensional bounded martingales $\left\{P_{t, p}^{\alpha}, \alpha\right\}$ and by re-writing $v$ as

$v(t, x, p)=\inf \left\{y: \exists(\nu, \alpha)\right.$ such that $Y_{t, x, y}^{\nu}(T) \geq \ell$ and $\left.\min _{i \leq \kappa}\left(\Delta^{i}\left(X_{t, x}(T), Y_{t, x, y}^{\nu}(T)\right)-P_{t, p}^{\alpha, i}(T)\right) \geq 0\right\}$,

where $\Delta^{i}(x, y):=\mathbf{1}_{\left\{y-g(x) \geq-\gamma^{i}\right\}}$ and $P_{t, p}^{\alpha, i}$ denotes the $i$-th component of $P_{t, p}^{\alpha}$. As in [6], "at the optimum" each process $P_{t, p}^{\alpha, i}$ has to be interpreted as the martingale coming from the martingale representation of $\mathbf{1}_{\left\{Y_{t, x, y}^{\nu}(T)-g\left(X_{t, x}(T)\right) \geq-\gamma^{i}\right\}}$.

The above reduction allows to appeal to the Geometric dynamic programming principle (GDPP) of Soner and Touzi [14], which leads to the PDE characterization stated in Theorem 2.1 below, with suitable boundary conditions.

We shall however see that both the associated Hamilton-Jacobi-Bellman operator and the boundary conditions are discontinuous, which leaves little hope to be able to establish a comparison result, and therefore build a convergent numerical scheme directly based on this PDE characterization.

We therefore introduce a sequence of approximating problems that are more regular and for which we can prove comparison. We show that they converge to the value function at any continuity point in the $p$-variable, or, more precisely, to its right and left limits in the $p$-variable, depending on the chosen approximating sequence.

In particular, we will show that it allows to approximate point-wise the relaxed problems:

$$
\underline{v}(t, x, p):=\inf \left\{y: \forall \varepsilon>0 \exists \nu^{\varepsilon} \text { s.t. } Y_{t, x, y}^{\nu^{\varepsilon}}(T) \geq \ell, \mathbb{P}\left[Y_{t, x, y}^{\nu^{\varepsilon}}(T)-g\left(X_{t, x}(T)\right) \geq-\gamma^{i}\right] \geq p^{i}-\varepsilon \forall i \leq \kappa\right\}
$$


and

$$
\bar{v}(t, x, p):=\inf \left\{y: \exists \nu \text { s.t. } Y_{t, x, y}^{\nu}(T) \geq \ell, \mathbb{P}\left[Y_{t, x, y}^{\nu}(T)-g\left(X_{t, x}(T)\right) \geq-\gamma^{i}\right]>p^{i} \quad \forall i \leq \kappa\right\} .
$$

The first value function $\underline{v}$ is indeed shown to be the left-limit in $p$ of $v$, while $\bar{v}$ is the right-limit in $p$ of $v$. In cases where $v$ is continuous, then $\bar{v}=v=\underline{v}$ and our schemes converge to the original value function. However the continuity of $v$ in its $p$-variable seems are a-priori difficult to prove by lack of convexity and strict monotonicity of the indicator function, and may fail in general. Still, one of the two approximations can be chosen to solve practical problems.

In this paper, we restrict to the case where the market is complete but the amount of money that can be invested in the risky assets is bounded. The incomplete market case could be discussed by following the lines of the paper, but will add extra complexity. Since the proofs below are already complex, we decided to restrict to the complete market case. The fact that the amount of money that can be invested in the risky assets is bounded could also be relaxed. It does not really simplifies the arguments. On the other hand, it is well-known that quantile hedging type strategies can lead to the explosion of the number of risky asset to hold in the portfolio near the maturity. This is due to the fact that it typically leads to hedging discontinuous payoffs, see the example of a call option in the Black-and-Scholes model in [11]. In our multiple constraint case, we expect to obtain a similar behavior. The constraint on the portfolio is therefore imposed to avoid this explosion, which leads to strategies that can not be implemented in practice.

The rest of the paper is organized as follows. The P\&L matching problem and its PDE characterization are presented in Section 2. In Section 3, we describe the sequence of approximating problems and the corresponding PDE characterizations. The proofs are collected in Section 4.

Notations: We denote by $\mathbb{M}^{n, d}$ the set of $n \times d$ matrices, Trace $[M]$ the trace of $M \in \mathbb{M}^{d, d}=: \mathbb{M}^{d}$ and $M^{\top}$ its transposition. The $i$-th line of $M \in \mathbb{M}^{n, d}$ is denoted by $M^{i}$. We identify $\mathbb{R}^{d}$ to $\mathbb{M}^{d, 1}$. For $x \in \mathbb{R}^{d}, B_{r}(x)$ is defined as the open ball of radius $r>0$ and center $x$, and $x_{I}:=\left(x_{i}\right)_{i \in I}$ for $I \subset\{1, . ., d\}$. For $x, y \in \mathbb{R}^{d}$, we write $x \geq y$ for $x^{i} \geq y^{i}$ for all $i \leq d$. We write diag $[x]$ to denote the diagonal matrix of $\mathbb{M}^{d}$ who $i$-th diagonal element is $x^{i}$. For a set $A \subset \mathbb{R} \times \mathbb{R}^{d}$, we note $\operatorname{int}(A)$ its interior, $\bar{A}$ its closure, $\partial A$ its boundary and $\partial_{T} A:=\left\{x \in \mathbb{R}^{d}:(T, x) \in \partial A\right\}$. Any inequality between random variables should be understood in the a.s. sense.

\section{PDE characterization of the P\&L matching problem}

\subsection{Problem formulation}

Let $W$ be a standard $d$-dimensional Brownian motion defined on a complete probability space $(\Omega, \mathcal{F}, \mathbb{P})$, with $d \geq 1$. We denote by $\mathbb{F}:=\left\{\mathcal{F}_{t}\right\}_{0 \leq t \leq T}$ the $\mathbb{P}$-complete filtration generated by $W$ on some time interval $[0, T]$ with $T>0$.

Given $(t, x) \in[0, T] \times(0, \infty)^{d}$, the stock price process $X_{t, x}$, starting from $x$ at time $t$, is assumed to be the unique strong solution of

$$
X(s)=x+\int_{t}^{s} \operatorname{diag}[X(r)] \mu(X(r)) d r+\int_{t}^{s} \operatorname{diag}[X(r)] \sigma(X(r)) d W_{r}
$$

where

$$
x \in(0, \infty)^{d} \mapsto \operatorname{diag}[x](\mu(x), \sigma(x))=:\left(\mu_{X}(x), \sigma_{X}(x)\right) \in \mathbb{R}^{d} \times \mathbb{M}^{d}
$$


is Lipschitz continuous and $\sigma$ is invertible. All over this paper, we shall assume that there exists some $L>0$ such that

$$
|\mu|+|\sigma|+\left|\sigma^{-1}\right| \leq L \text { on }(0, \infty)^{d}
$$

A financial strategy is described by an element $\nu$ of the set $\mathcal{U}$ of progressively measurable processes taking values in some fixed subset $U \subset \mathbb{R}^{d}$, each component $\nu_{r}^{i}$ at time $r$ representing the amount of money invested in the $i$-th risky asset $r$. Importantly, we shall assume all over this paper that

$$
U \text { is convex closed, its interior contains } 0 \text { and } \sup \{|u|, u \in U\} \leq L .
$$

This (important) assumption will be commented in Remarks 2.1 below. In the above, we label by $L$ the different bounds because this constant will be used hereafter.

For sake of simplicity, we assume that the risk free interest rate is equal to zero. The associated wealth process, starting with the value $y$ at time $t$, is thus given by

$$
Y(s)=y+\int_{t}^{s} \nu_{r}^{\top} \operatorname{diag}\left[X_{t, x}(r)\right]^{-1} d X_{t, x}(r)=y+\int_{t}^{s} \mu_{Y}\left(X_{t, x}(r), \nu_{r}\right) d r+\int_{t}^{s} \sigma_{Y}\left(X_{t, x}(r), \nu_{r}\right) d W_{r}
$$

where

$$
\mu_{Y}(x, u):=u^{\top} \mu(x) \text { and } \sigma_{Y}(x, u):=u^{\top} \sigma(x),(x, u) \in \mathbb{R}_{+}^{d} \times U .
$$

The aim of the trader is to hedge an European option of payoff $g\left(X_{t, x}(T)\right)$ at time $T$, where

$$
g:(0, \infty)^{d} \mapsto \mathbb{R} \text { is Lipschitz continuous. }
$$

Here, the price is chosen so that the net wealth $Y_{t, x, y}^{\nu}(T)-g\left(X_{t, x}(T)\right)$ satisfies a P\&L constraint. Namely, given a collection of thresholds $\gamma:=\left(\gamma^{i}\right)_{i \leq \kappa} \in \mathbb{R}^{\kappa}$ and of probabilities $\left(p^{i}\right)_{i \leq \kappa} \in[0,1]^{\kappa}$, for some $\kappa \geq 1$, the price of the option is defined as the minimal initial wealth $y$ such that there exists a strategy $\nu \in \mathcal{U}$ satisfying

$$
\mathbb{P}\left[Y_{t, x, y}^{\nu}(T) \geq g^{i}\left(X_{t, x}(T)\right)\right] \geq p^{i} \text { for all } i \in\{1, \ldots, \kappa\}=: \mathcal{K},
$$

where

$$
g^{i}:=g-\gamma^{i}, i \in \mathcal{K} .
$$

Obviously, we can assume without loss of generality that

$$
0 \leq \gamma^{1} \leq \gamma^{2} \leq \cdots \leq \gamma^{\kappa}
$$

This means that the net hedging loss should not exceed $-\gamma^{i}$ with probability more than $p^{i}$. This coincides with a constraint on the distribution of the P\&L of the trader, in the sense that it should match the constraints imposed by the discrete histogram associated to $(\gamma, p)$. In order to avoid that the wealth process goes too negative, even with small probability, we further impose that $Y_{t, x, y}^{\nu}(T) \geq \ell$ for some $\ell \in \mathbb{R}_{-}$. The price is then defined, for $(t, x, p) \in[0, T] \times(0, \infty)^{d} \times[0,1]^{\kappa}$, as:

$$
v(t, x, p):=\inf \left\{y \geq \ell: \exists \nu \in \mathcal{U} \text { s.t } Y_{t, x, y}^{\nu}(T) \geq \ell \text { and } \mathbb{P}\left[Y_{t, x, y}^{\nu}(T) \geq g^{i}\left(X_{t, x}(T)\right)\right] \geq p^{i} \text { for } i \in \mathcal{K}\right\} .
$$

Note that, after possibly changing $g$ and $\gamma$, one can always reduce to the case where

$$
g^{1} \geq g^{2} \geq \cdots \geq g^{\kappa} \geq \ell .
$$

We further assume that $g$ is bounded from above and that $g^{\kappa}>\ell$ uniformly, which, after possibly changing the constant $L$ can be written as

$$
\ell+L^{-1} \leq g^{\kappa} \leq g \leq L
$$


Remark 2.1 The above criteria extends the notion of quantile hedging discussed in [11] to multiple constraints in probability. In [11], it is shown that the optimal strategy associated to a quantile hedging problem may lead to the hedging of a discontinuous payoff. This is in particular the case in the Black and Scholes model when one wants to hedge a call option, only with a given probability of success. This typical feature is problematic in practice as it leads to a possible explosion of the delta near the maturity. This explains why we have deliberately imposed that $U$ is compact, i.e. that the amount of money invested in the stocks is bounded.

Remark 2.2 Since $U$ is bounded, see $(2.3), Y_{t, x, y}^{\nu}$ is a $\mathbb{Q}_{t, x}$-martingale for $\mathbb{Q}_{t, x} \sim \mathbb{P}$ defined by

$$
\frac{d \mathbb{Q}_{t, x}}{d \mathbb{P}}=\mathcal{E}\left(\int_{t}^{\cdot}-\left(\mu \sigma^{-1}\right)\left(X_{t, x}(s)\right) d W_{s}\right)_{T},
$$

recall (2.2). The constraint $Y_{t, x, y}^{\nu}(T) \geq \ell$ thus implies that $Y_{t, x, y}^{\nu} \geq \ell$ on $[t, T]$. In particular, the restriction to $y \geq \ell$ is redundant. We write it only for sake of clarity.

\subsection{Problem reduction and domain decomposition}

As in [6], the first step consists in converting our stochastic target problem under probability constraints into a stochastic target problem in standard form as studied in [15]. This will allow us to appeal to the Geometric Dynamic Programming Principle to provide a PDE characterization of $v$. In our context, such a reduction is obtained by adding a family of $\kappa$-dimensional martingales defined by

$$
P_{t, p}^{\alpha}(s)=p+\int_{t}^{s} \alpha_{r} d W_{r},(t, p, \alpha) \in[0, T] \times[0,1]^{\kappa} \times \mathcal{A},
$$

where $\mathcal{A}$ is the set of predictable processes $\alpha$ in $L^{2}\left([0, T], \mathbb{M}^{\kappa, d}\right)$. Given $(t, p) \in[0, T] \times[0,1]^{\kappa}$, we further denote by $\mathcal{A}_{t, p}$ the set of elements $\alpha \in \mathcal{A}$ such that $P_{t, p}^{\alpha} \in[0,1]^{\kappa} d t \times d \mathbb{P}$-a.e. on $[t, T]$ and define

$$
G(x, p):=\inf \left\{y \geq \ell: \min _{i \in \mathcal{K}}\left\{1_{\left\{y \geq g^{i}(x)\right\}}-p_{i}\right\} \geq 0\right\},(x, p) \in(0, \infty)^{d} \times \mathbb{R}^{\kappa} .
$$

Note that

$$
G(\cdot, p)=\infty \text { for } p \notin(-\infty, 1]^{\kappa} \text {, and } G\left(\cdot, p_{1}\right) \geq G\left(\cdot, p_{2}\right) \text { if } p_{1}^{i} \vee 0 \geq p_{2}^{i} \forall i \in \mathcal{K} .
$$

Proposition 2.1 For all $(t, x, p) \in[0, T] \times(0, \infty)^{d} \times[0,1]^{\kappa}$,

$$
\begin{aligned}
v(t, x, p) & =\inf \left\{y \geq \ell: Y_{t, x, y}^{\nu}(T) \geq G\left(X_{t, x}(T), P_{t, p}^{\alpha}(T)\right) \text { for some }(\nu, \alpha) \in \mathcal{U} \times \mathcal{A}_{t, p}\right\}, \\
& =\inf \left\{y \geq \ell: Y_{t, x, y}^{\nu}(T) \geq G\left(X_{t, x}(T), P_{t, p}^{\alpha}(T)\right) \text { for some }(\nu, \alpha) \in \mathcal{U} \times \mathcal{A}\right\}
\end{aligned}
$$

Proof. The proof follows from the same arguments as in [6]. We provide it for completeness. We fix $(t, x, p) \in[0, T] \times(0, \infty)^{d} \times[0,1]^{\kappa}$, set $v:=v(t, x, p)$ for ease of notations, and denote by $w_{1}$ and $w_{2}$ the right-hand side of (2.13) and (2.14) respectively. The fact that $w_{1} \geq w_{2}$ is obvious. Conversely, if $Y_{t, x, y}^{\nu}(T) \geq G\left(X_{t, x}(T), P_{t, p}^{\alpha}(T)\right)$ for some $(\nu, \alpha) \in \mathcal{U} \times \mathcal{A}$, then $(2.12)$ implies that $P_{t, p}^{\alpha, i}(T) \leq 1$ for all $i \in \mathcal{K}$. Since $P_{t, p}^{\alpha}$ is a martingale, it takes values in $(-\infty, 1]^{\kappa}$ on $[t, T]$. Moreover, we can find $\tilde{\alpha} \in \mathcal{A}$ such that $P_{t, p}^{\tilde{\alpha}, i}(T)=0$ on $A_{i}:=\left\{\min _{[t, T]} P_{t, p}^{\alpha, i} \leq 0\right\}$ and $P_{t, p}^{\tilde{\alpha}, i}(T)=P_{t, p}^{\alpha, i}(T)$ on $A_{i}^{c}$ for $i \in \mathcal{K}$. It follows from the above discussion and the martingale property of $P_{t, p}^{\tilde{\alpha}}$ that it takes values in $[0,1]^{\kappa}$ on $[t, T]$, so that $\tilde{\alpha} \in \mathcal{A}_{t, p}$. Since $P_{t, p}^{\tilde{\alpha}, i}(T) \leq P_{t, p}^{\alpha, i}(T) \vee 0$, the inequality $Y_{t, x, y}^{\nu}(T) \geq G\left(X_{t, x}(T), P_{t, p}^{\alpha}(T)\right)$ together with (2.12) imply that $Y_{t, x, y}^{\nu}(T) \geq G\left(X_{t, x}(T), P_{t, p}^{\tilde{\alpha}}(T)\right)$. This shows that $w_{2} \geq w_{1}$, so that $w_{2}=w_{1}$. It remains to show that $v=w_{2}$. The inequality $w_{2} \geq v$ is an immediate consequence of the martingale property of $P_{t, p}^{\alpha}$. On the other hand, for $y>v$, we can find $\nu \in \mathcal{U}$ such that $\bar{p}^{i}:=\mathbb{P}\left[Y_{t, x, y}^{\nu}(T) \geq\right.$ 
$\left.g^{i}\left(X_{t, x}(T)\right)\right] \geq p^{i}$ for all $i \in \mathcal{K}$. Set $\bar{p}:=\left(\bar{p}^{i}\right)_{i \in \mathcal{K}}$. Then, the martingale representation theorem implies that we can find $\alpha \in \mathcal{A}$ such that $P_{t, \bar{p}}^{\alpha, i}(T)=\mathbf{1}_{\left\{Y_{t, x, y}^{\nu}(T) \geq g^{i}\left(X_{t, x}(T)\right)\right\}}$ for each $i \in \mathcal{K}$. We conclude by observing that $P_{t, \bar{p}}^{\alpha, i}(T) \geq P_{t, p}^{\alpha, i}(T)$ for each $i \in \mathcal{K}$.

Remark 2.3 As in [6], the new controlled process $P_{t, p}^{\alpha}$ should be interpreted as the martingale with components given by $\left(\mathbb{P}\left[Y_{t, x, y}^{\nu}(T) \geq g^{i}\left(X_{t, x}(T)\right) \mid \mathcal{F}_{s}\right]\right)_{s \in[t, T]}$, at least when the controls $\nu$ and $\alpha$ are optimal. This is rather transparent in the above proof. The fact that we can restrict to the set of controls $\mathcal{A}_{t, p}$ is therefore clear since a conditional probability should take values in $[0,1]$.

Remark 2.4 Note that $\alpha \in \mathcal{A}_{t, p}$ implies that $\alpha^{i \cdot} \equiv 0$ for all $i \in \mathcal{K}$ such that $p^{i} \in\{0,1\}$, since $P_{t, p}^{\alpha}$ is a martingale.

The representation (2.14) coincides with a stochastic target problem in standard form but with unbounded controls as studied in [6], unbounded referring to the fact that $\alpha$ can not be bounded a-priori since it comes from the martingale representation theorem. In particular, a PDE characterization of the value function $v$ in the parabolic interior of the domain

$$
D:=[0, T) \times(0, \infty)^{d} \times(0,1)^{\kappa}
$$

follows from the general results of [6]. The main difference comes from the fact that the constraints $P^{\alpha, i} \in[0,1]$ introduce boundary conditions that have to be discussed separately. In order to deal with these boundary conditions, we first divide the closure of the domain $\bar{D}$ into different regions corresponding to its parabolic interior $D$ and the different boundaries associated to the level of conditional probabilities. Namely, given

$$
\mathcal{P}_{\kappa}:=\left\{(I, J) \in \mathcal{K}^{2}: I \cap J=\emptyset \text { and } I \cup J \subset \mathcal{K}\right\},
$$

we set, for $(I, J) \in \mathcal{P}_{\kappa}$,

$$
D_{I J}:=[0, T) \times(0, \infty)^{d} \times B_{I J},
$$

where

$$
B_{I J}:=\left\{p \in[0,1]^{\kappa}: p^{i}=0 \text { for } i \in I, p^{j}=1 \text { for } j \in J, \text { and } 0<p^{l}<1 \text { for } l \notin I \cup J\right\}
$$

Then,

$$
[0, T) \times(0, \infty)^{d} \times[0,1]^{\kappa}=\cup_{(I, J) \in \mathcal{P}_{\kappa}} D_{I J} .
$$

The interpretation of the partition is the following. For $(t, x, p) \in D$, any $p^{i}$ takes values in $(0,1)$ so that $P_{t, p}^{\alpha, i}$ is not constrained locally at time $t$ by the state constraints which appears in (2.13), namely $P_{t, p}^{\alpha, i} \in[0,1]$. This means that the control $\alpha^{i \cdot}$ can be chosen arbitrarily, at least locally around the initial time $t$. When $(t, x, p) \in D_{I J}$ with $I \cup J \neq \emptyset$, then there is at least one $i \leq \kappa$ such that $p^{i}=0$ or $p^{i}=1$. In this case the state constraints $P_{t, p}^{\alpha, i} \in[0,1]$ on $[t, T]$ imposes to choose $\alpha^{i \cdot}=0$ on $[t, T]$, see Remark 2.4. Hence, letting $\pi_{I J}$ be the operator defined by

$$
p \in[0,1]^{\kappa} \mapsto \pi_{I J}(p)=\left(p^{i} \mathbf{1}_{i \notin I \cup J}+\mathbf{1}_{i \in J}\right)_{i \in \mathcal{K}}
$$

for $(I, J) \in \mathcal{P}_{\kappa}$, we have

$$
v=v_{I J}:=v\left(\cdot, \pi_{I J}(\cdot)\right) \text { on } \bar{D}_{I J},
$$

where, for $(t, x, p) \in \bar{D}$,

$$
v_{I J}(t, x, p)=\inf \left\{y \geq \ell: Y_{t, x, y}^{\nu}(T) \geq G\left(X_{t, x}(T), P_{t, \pi_{I J}(p)}^{\alpha}(T)\right) \text { for some }(\nu, \alpha) \in \mathcal{U} \times \mathcal{A}_{t, \pi_{I J}(p)}^{I J}\right\}
$$


with

$$
\mathcal{A}_{t, \pi_{I J}(p)}^{I J}:=\left\{\alpha \in \mathcal{A}_{t, p}: \alpha^{i \cdot}=0 \text { for all } i \in I \cup J\right\}
$$

recall Remark 2.4.

In the rest of the paper, we shall write $(I, J) \in \mathcal{P}_{\kappa}^{k}$ when $(I, J) \in \mathcal{P}_{\kappa}$ and $|I|+|J|=k, k \leq \kappa$. We shall also use the notations $\left(I^{\prime}, J^{\prime}\right) \supset(I, J)$ when $I^{\prime} \supset I$ and $J^{\prime} \supset J$. If in addition, $\left(I^{\prime}, J^{\prime}\right) \neq(I, J)$, then we will write $\left(I^{\prime}, J^{\prime}\right) \supsetneq(I, J)$.

Remark 2.5 It is clear that $v$ and each $v_{I J},(I, J) \in \mathcal{P}_{\kappa}$, are non-decreasing with respect to their $p$-parameter. In particular, $v_{I J^{\prime}} \geq v_{I J} \geq v_{I^{\prime} J}$ for $\left(I^{\prime}, J^{\prime}\right) \supset(I, J)$.

Remark 2.6 Since $g^{i} \geq g^{j}$ for $i \leq j$, it would be natural to restrict to the case where $p^{i} \leq p^{j}$ for $i \leq j$. From the PDE point of view, this would lead to the introduction of boundary conditions on the planes for which $p^{i}=p^{j}$ for some $i \neq j$. Since this restriction does not appear to be necessary in our approach, we deliberately do not use this formulation. From the pure numerical point of view, one could however use the fact that $v(\cdot, p)=v(\cdot, \hat{p})$ where $\hat{p}$ is defined by $\hat{p}^{j}=\max _{i \leq j} p^{j}$ for $i \leq \kappa$.

Remark 2.7 Note that, as defined above on $\bar{D}_{I J}$, the function $v_{I J}$ depends on its $p$-parameters only through the components $\left(p^{l}\right)_{l \notin I \cup J}$. However, for ease of notations, we shall always use the notation $v_{I J}(\cdot, p)$ instead of a more transparent notation such as $v_{I J}\left(t, x,\left(p^{l}\right)_{l \notin I \cup J}\right)$. Similarly, a test function on $\bar{D}_{I J}$ depends on the $p$-parameter only through $\left(p^{l}\right)_{l \notin I \cup J}$.

Remark 2.8 Note that, for any $J \subset \mathcal{K}$,

$$
v_{J^{c} J}=\inf \left\{y \geq \ell: Y_{t, x, y}^{\nu}(T) \geq g_{J}\left(X_{t, x}(T)\right) \text { for some } \nu \in \mathcal{U}\right\}
$$

where

$$
g_{J}:=\max _{j \in J} g^{j} \vee \ell
$$

coincides with the super-hedging price of the payoff $g_{J}\left(X_{t, x}(T)\right)$, while

$$
v_{\mathcal{K} \emptyset}=\inf \left\{y \geq \ell: \mathbf{1}_{\left\{Y_{t, x, y}^{\nu}(T) \geq \max _{i \leq \kappa} g^{i}\left(X_{t, x}(T)\right)\right\}} \geq 0 \text { and } Y_{t, x, y}^{\nu}(T) \geq \ell \text { for some } \nu \in \mathcal{U}\right\}=\ell .
$$

\subsection{PDE characterization}

As already mentioned, stochastic target problems of the form (2.18) have been studied in [6] which provides a PDE characterization of each value function $v_{I J}$ on $D_{I J}$. In order to state it, we first need to introduce some additional notations. For ease of notations, we set

$$
\mu_{X, P}:=\left(\begin{array}{c}
\mu_{X} \\
0_{\kappa}
\end{array}\right) \text { and } \sigma_{X, P}(\cdot, a):=\left(\begin{array}{c}
\sigma_{X} \\
a
\end{array}\right) \text { for } a \in \mathbb{M}^{\kappa, d},
$$

where $0_{\kappa}:=(0, \ldots, 0) \in \mathbb{R}^{\kappa}$.

Given $(I, J) \in \mathcal{P}_{\kappa}$ and $\varepsilon>0$, we then define

$$
F_{I J}^{\epsilon}:=\sup _{(u, a) \in N_{I J}^{\epsilon}} L^{u, a}
$$

where, for $(u, a) \in U \times \mathbb{M}^{\kappa, d}$ and $\theta:=(x, q, Q) \in \Theta:=(0, \infty)^{d} \times \mathbb{R}^{d+\kappa} \times \mathbb{M}^{d+\kappa, d+\kappa}$,

$$
L^{u, a}(\theta):=\mu_{Y}(x, u)-\mu_{X, P}(x)^{\top} q-\frac{1}{2} \operatorname{Trace}\left[\left(\sigma_{X, P} \sigma_{X, P}^{\top}\right)(x, a) Q\right],
$$


and

$$
N_{I J}^{\epsilon}:=\left\{(u, a) \in U \times A_{I J}:\left|\mathcal{N}^{u, a}\right| \leq \epsilon\right\}
$$

with

$$
\mathcal{N}^{u, a}(x, q):=\sigma_{Y}(x, u)-q^{\top} \sigma_{X, P}(x, a) \text { and } A_{I J}:=\left\{a \in \mathbb{M}^{\kappa, d}: a^{k \cdot}=0 \text { for } k \in I \cup J\right\} .
$$

The main result of [6] states that $v_{I J}$ is a discontinuous viscosity solution of the PDE

$$
\min \left\{\varphi-\ell,-\partial_{t} \varphi+F_{I J}^{0}\left(\cdot, D \varphi, D^{2} \varphi\right)\right\}=0 \text { on } D_{I J}
$$

where, for a smooth function $\varphi:(t, x, p) \in[0, T] \times \mathbb{R}^{d} \times \mathbb{R}^{\kappa}, D \varphi$ and $D^{2} \varphi$ stand for the gradient and the Hessian matrix with respect to $(x, p)$, and $\partial_{t} \varphi$ stands for the time derivative. The label "discontinuous viscosity solution" means that it has be stated in terms of the upper- and lower-semicontinuous envelopes of $v$, see Definition 2.2 below, and that we need to relax the operator $F_{I J}^{0}$, which may not be continuous, by considering the upper- and lower-semicontinuous envelopes $F_{I J}^{*}$ and $F_{I J *}$

$$
F_{I J}^{*}(\theta):=\limsup _{\substack{\left(\theta^{\prime}, \varepsilon^{\prime}\right) \rightarrow(\theta, 0) \\\left(\theta^{\prime}, \varepsilon^{\prime}\right) \in \Theta \times \mathbb{R}_{+}}} F_{I J}^{\varepsilon^{\prime}}\left(\theta^{\prime}\right) \text { and } F_{I J *}(\theta):=\liminf _{\substack{\left(\theta^{\prime}, \varepsilon^{\prime}\right) \rightarrow(\theta, 0) \\\left(\theta^{\prime}, \varepsilon^{\prime}\right) \in \Theta \times \mathbb{R}_{+}}} F_{I J}^{\varepsilon^{\prime}}\left(\theta^{\prime}\right) .
$$

This leads to a system, hereafter called (S), of PDEs, each stated on a sub-domain $D_{I J}$, with appropriate boundary conditions, see Theorem 2.2 and Corollary 2.1 below.

Before defining precisely what we mean by a solution of (S), we need to introduce an extra technical object to which we will appeal when we define the notion of subsolution.

Definition 2.1 Given $(I, J) \in \mathcal{P}_{\kappa}$ and $(t, x, p) \in D_{I J}$, we denote by $\mathcal{C}_{I J}(t, x, p)$ the set of $C^{1,2}$ functions $\varphi$ with the following property: for all $\varepsilon>0$, all open set $B$ such that $(x, D \varphi(t, x, p)) \in B$ and $N_{I J}^{0} \neq \emptyset$ on $B$, and all $(\tilde{u}, \tilde{a}) \in N_{I J}^{0}(x, D \varphi(t, x, p))$, there exists an open neighborhood $B^{\prime}$ of $(x, D \varphi(t, x, p))$ and a locally Lipschitz map $(\hat{u}, \hat{a})$ such that $|(\hat{u}, \hat{a})(x, D \varphi(t, x, p))-(\tilde{u}, \tilde{a})| \leq \varepsilon$ and $(\hat{u}, \hat{a}) \in N_{I J}^{0}$ on $B^{\prime}$.

Remark 2.9 Fix $(I, J) \in \mathcal{P}_{\kappa}$ such that there exists $i \in \mathcal{K} \backslash(I \cup J)$. Let $\varphi$ be a smooth function such that $D_{p^{i}} \varphi \neq 0$ on a neighborhood of $(t, x, p) \in \bar{D}$. Then, $(u, a) \in N_{I J}^{0}(x, D \varphi(t, x, p))$ is equivalent to

$$
a^{i \cdot}=\left(\sigma_{Y}(x, u)-D_{x} \varphi(t, x, p)^{\top} \sigma_{X}(x)-\sum_{j \notin I \cup J \cup\{i\}} a^{j \cdot} D_{p^{j}} \varphi(t, x, p)\right) / D_{p^{i}} \varphi(t, x, p) .
$$

Since $D_{p^{i}} \varphi \neq 0$ on a neighborhood of $(t, x, p)$, this readily implies that $\varphi \in C_{I J}(t, x, p)$.

A viscosity solution of ( $\mathrm{S})$ is then defined as follows.

Definition 2.2 (i) Given a locally bounded map $V$ defined on $\bar{D}$ and $(I, J) \in \mathcal{P}_{\kappa}$, we define $V_{I J}:=$ $V\left(\cdot, \pi_{I J}(\cdot)\right)$ and

$$
V_{I J}^{*}(t, x, p):=\limsup _{\substack{\left.t^{\prime}, x^{\prime}, p^{\prime}\right) \rightarrow(t, x, p) \\\left(t^{\prime}, x^{\prime}, p^{\prime}\right) \in D_{I J}}} V_{I J}\left(t^{\prime}, x^{\prime}, p^{\prime}\right) \text { and } V_{I J *}(t, x, p):=\liminf _{\substack{\left(t^{\prime}, x^{\prime}, p^{\prime}\right) \rightarrow(t, x, p) \\\left(t^{\prime}, x^{\prime}, p^{\prime}\right) \in D_{I J}}} V_{I J}\left(t^{\prime}, x^{\prime}, p^{\prime}\right),
$$

for $(t, x, p) \in D_{I J}$.

(ii) We say that $V$ is a discontinuous viscosity supersolution of $(\mathrm{S})$ if $V_{I J *}$ is a viscosity supersolution of

$$
\min \left\{\varphi-\ell,-\partial_{t} \varphi+F_{I J}^{*}\left(\cdot, D \varphi, D^{2} \varphi\right)\right\}=0 \text { on } D_{I J}
$$


for each $(I, J) \in \mathcal{P}_{\kappa}$.

(iii) We say that $V$ is a discontinuous viscosity subsolution of $(\mathrm{S})$ if $V_{I J}^{*}$ is a viscosity subsolution of

$$
\min \left\{\varphi-\ell,-\partial_{t} \varphi+F_{I J *}\left(\cdot, D \varphi, D^{2} \varphi\right)\right\}=0 \text { if } \varphi \in \mathcal{C}_{I J} \text {, on } D_{I J},
$$

for each $(I, J) \in \mathcal{P}_{\kappa}$.

(iv) We say that $V$ is a discontinuous viscosity solution of $(\mathrm{S})$ if it is both a discontinuous super-and subsolution of (S).

We can now state our first result which is a direct Corollary of Theorem 2.1 in [6].

Theorem 2.1 The function $v$ is a discontinuous viscosity solution of $(\mathrm{S})$.

Proof. The above result is an immediate consequence of Theorem 2.1 in [6]. Note that we replaced their condition Assumption 2.1 by the condition $\varphi \in \mathcal{C}_{I J}$, which is equivalent, in the statement of the subsolution property, see Remark 2.9 .

Remark 2.10 Fix $(I, J) \in \mathcal{P}_{\kappa}$ such that $I \cup J=\mathcal{K}$. Then, $(u, a) \in N_{I J}^{\varepsilon}(x, D \varphi(t, x, p))$ implies that

$$
\left|u^{\top} \sigma(x)-D_{x} \varphi(t, x, p)^{\top} \operatorname{diag}[x] \sigma(x)\right| \leq \varepsilon .
$$

Since $u \in U$ and $\sigma(x)$ is invertible by assumption, one easily checks that (2.20) implies

$$
\operatorname{diag}[x] D_{x} \varphi(t, x, p) \in U,
$$

recall the usual convention $\sup \emptyset=-\infty$. This is the classical gradient constraint that appears in super-hedging problems with constraints on the strategy, see e.g. [9], where it is written in terms of proportions of the wealth invested in the risky assets.

Remark 2.11 Let $\varphi$ be a smooth function. If $D_{p^{i}} \varphi(t, x, p)=0$ for $i \notin I \cup J$, then $N_{I J}^{\varepsilon}(x, D \varphi(t, x, p))$ takes the form $U_{\varepsilon} \times \mathbb{M}^{\kappa, d}$ for some $U_{\varepsilon} \subset U, \varepsilon>0$. Thus the optimization over $a \in \mathbb{M}^{\kappa, d}$ in the definition of $F_{I J}^{\varepsilon}$ is performed over an unbounded set. On the other hand, if $D_{p^{i}} \varphi(t, x, p)>0$ for $i \notin I \cup J$, then the same arguments as in Remark 2.9 imply that at least one line of $a$ is given by the other ones. In particular, for $|I|+|J|=\kappa-1$, the sequence of sets $\left(N_{I J}^{\varepsilon}(x, D \varphi(t, x, p))\right)_{0 \leq \varepsilon \leq 1}$ is contained in a compact subset of $U \times \mathbb{M}^{\kappa, d}$. This implies that $F_{I J}^{*} \neq F_{I J *}$ in general.

As already mentioned the main difficulty comes from the boundary conditions. We first state the space boundary condition in the $p$-variable.

Theorem 2.2 Fix $(I, J),\left(I^{\prime}, J^{\prime}\right) \in \mathcal{P}_{\kappa}$ such that $\left(I^{\prime}, J^{\prime}\right) \supset(I, J)$, we have

(i) $v_{I J *}$ is viscosity supersolution of

$$
\min \left\{\varphi-\ell,-\partial_{t} \varphi+F_{I J^{\prime}}^{*}\left(\cdot, D \varphi, D^{2} \varphi\right)\right\}=0 \text { on } D_{I J^{\prime}}
$$

(ii) $v_{I J}^{*}(t, x, p) \leq v_{I^{\prime} J^{\prime}}^{*}(t, x, p)$, for $(t, x, p) \in \bar{D}_{I J} \cap[0, T] \times(0, \infty)^{d} \times B_{I^{\prime} J^{\prime}}$.

Proof. It is proved by the same arguments as in the proofs of Proposition 4.2 and Proposition 4.4 below.

We now discuss the boundary condition as $t$ approaches $T$.

In the case where $I \cup J=\mathcal{K}$ with $|J|>0$, the map $v_{I J}$ coincides with the super-hedging problem associated to the payoff $g_{J}$ as defined in (2.19), recall Remark 2.8. One could therefore expect that 
$v_{I J}(T-, \cdot)=g_{J}$. However, as usual, see e.g. [9], the terminal condition for $v_{I J}$ is not the natural one since the gradient constraint that appears implicitly in (2.21), see Remark 2.10, should propagate up to the time boundary. The natural boundary condition should be given by the smallest function $\phi$ above $g_{J}$ that satisfies the associated gradient constraint $\operatorname{diag}[x] D_{x} \phi \in U$. This leads to the introduction of the "face-lifted" version of $g_{J}$ defined by:

$$
\hat{g}_{J}(x):=\sup _{\zeta \in \mathbb{R}^{d}}\left[g_{J}\left(x e^{\zeta}\right)-\delta_{U}(\zeta)\right]
$$

where

$$
\delta_{U}(\zeta):=\sup _{u \in U} u^{\top} \zeta, \zeta \in \mathbb{R}^{d}
$$

is the support function of the convex closed set $U$ and $x e^{\zeta}=\left(x^{i} e^{\zeta^{i}}\right)_{i \leq d}$.

When $I \cup J \neq \mathcal{K}$, the above mentioned gradient constraint does not appear anymore in (2.21), see e.g. Remark 2.9, and the terminal boundary condition can be naturally stated in terms of

$$
\begin{aligned}
\hat{G}(x, p) & :=\inf \left\{y \geq \ell: y \geq g^{i}(x) \mathbf{1}_{0<p^{i}<1}+\hat{g}^{i}(x) \mathbf{1}_{p^{i}=1}, \text { for all } i \in \mathcal{K}\right\} \\
& =\max _{i \in \mathcal{K}}\left(\ell \mathbf{1}_{p^{i}=0}+g^{i}(x) \mathbf{1}_{0<p^{i}<1}+\hat{g}^{i}(x) \mathbf{1}_{p^{i}=1}\right) .
\end{aligned}
$$

Corollary $2.1 v_{*}(T, \cdot) \geq \hat{G}_{*}$ and $v^{*}(T, \cdot) \leq \hat{G}^{*}$ on $(0, \infty)^{d} \times[0,1]^{\kappa}$.

Proof. It is a consequence of Proposition 3.2 and Theorem 3.1 below.

Remark 2.12 In the case of $\kappa=1$ and $g^{1} \geq \ell=0$, it is shown in [11] and [6] that the terminal condition should be face-lifted with respect to the $p$-variable when the set $U$ in which controls take values is $\mathbb{R}^{d}$. This follows from the convexity of the value function in its $p$-variable. Namely, the terminal condition as $t \rightarrow T$ is then given by $p^{1} g^{1}$. Corollary 2.1 shows that it is no more the case when we restrict to a compact set $U$.

Remark 2.13 Combining Theorem 2.1, Theorem 2.2 and Corollary 2.1 provides a PDE characterization of the value function $v$. However, the following should be noted:

1. It is clear that $\hat{G}_{*}<\hat{G}^{*}$ for some $p \in \partial[0,1]^{\kappa}$.

2. The boundary conditions induced by Theorem 2.2 may not lead to $v_{I J *} \geq v_{I J}^{*}$ on the boundary in the $p$-variable.

3. The operator $F_{I J}$ in (2.20) and (2.21) is in general discontinuous when $I \cup J \neq \mathcal{K}$, see Remark 2.11 above.

This prevents us from proving a general comparison result for super- and sub-solutions of (S). We are therefore neither able to prove that $v$ is the unique solution of $(\mathrm{S})$ in a suitable class, nor to prove the convergence of standard finite difference numerical schemes. In order to surround this difficulty, we shall introduce in the following Section a sequence of convergent approximating problems which are more regular and for which convergent schemes can be constructed. 


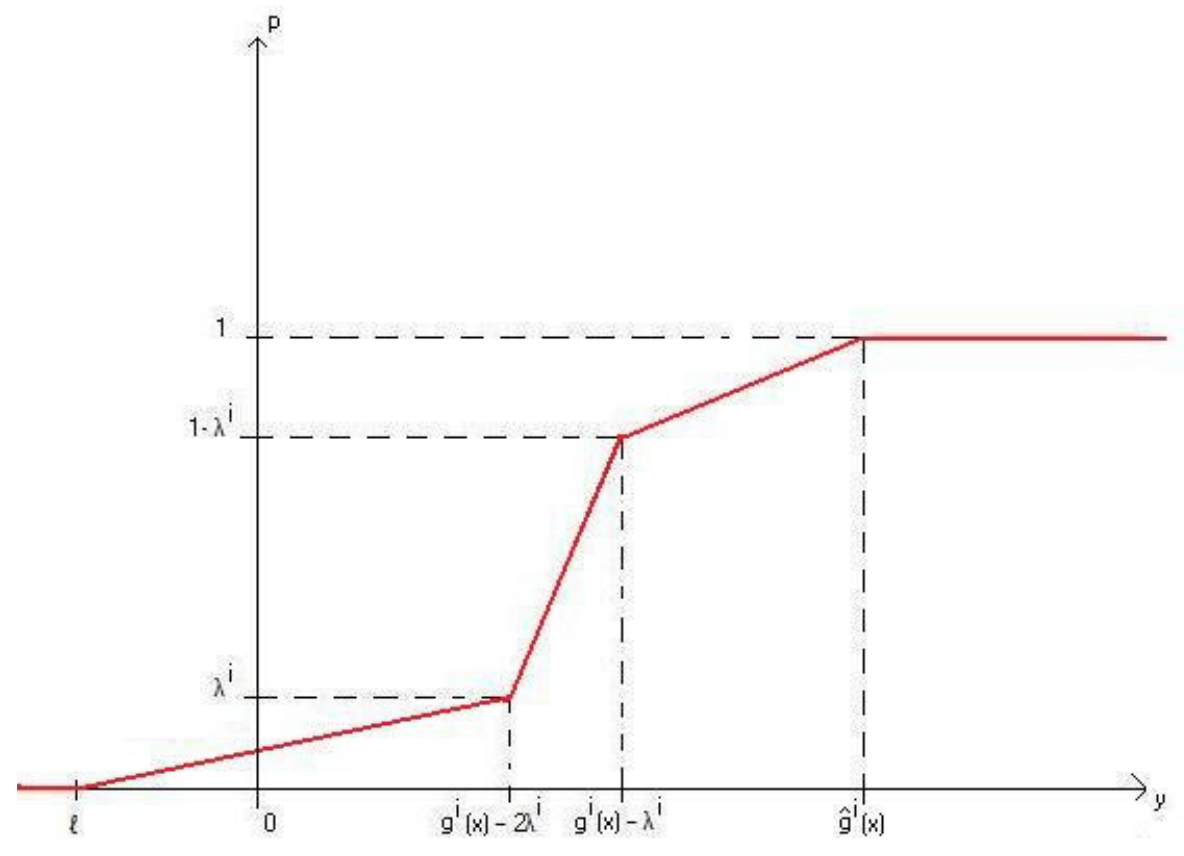

Figure 1: Function $\Delta_{\lambda}^{i}(x, \cdot)$

\section{The approximating problems}

\subsection{Definition and convergence properties}

Set $\Lambda:=\left(0,\left(L^{-1} \wedge 1\right) / 2\right)^{\kappa}$. Our approximating sequence $\left(v^{\lambda}\right)_{\lambda \in \Lambda}$ is a sequence of value functions associated to regularized stochastic target problems with controlled loss. Namely, for $\lambda \in \Lambda$, we set

$$
v^{\lambda}(t, x, p):=\inf \left\{y \geq \ell: \exists \nu \in \mathcal{U} \text { s.t } Y_{t, x, y}^{\nu}(T) \geq \ell \text { and } \mathbb{E}\left[\Delta_{\lambda}^{i}\left(X_{t, x}(T), Y_{t, x, y}^{\nu}(T)\right)\right] \geq p^{i} \text { for } i \in \mathcal{K}\right\},
$$

where

$$
\Delta_{\lambda}^{i}(x, y)=\left\{\begin{array}{ccc}
0 & \text { if } & y<\ell \\
\frac{\lambda^{i}(y-\ell)}{g^{i}(x)-2 \lambda^{i}-\ell} & \text { if } & \ell \leq y<g^{i}(x)-2 \lambda^{i} \\
\lambda^{i}+\frac{\left(1-2 \lambda^{i}\right)\left(y-g^{i}(x)+2 \lambda^{i}\right)}{\lambda^{i}} & \text { if } & g^{i}(x)-2 \lambda^{i} \leq y<g^{i}(x)-\lambda^{i} \\
\left(1-\lambda^{i}\right)+\frac{\lambda^{i}\left(y-g^{i}(x)+\lambda^{i}\right)}{\hat{g}^{i}(x)-g^{i}(x)+\lambda^{i}} & \text { if } & g^{i}(x)-\lambda^{i} \leq y<\hat{g}^{i}(x) \\
1 & \text { if } & y \geq \hat{g}^{i}(x)
\end{array}\right.
$$

is well-defined for $\lambda \in \Lambda=\left(0,\left(L^{-1} \wedge 1\right) / 2\right)^{\kappa}$ as in Figure 1, recall (2.7), (2.8) and (2.11).

The convergence $\left(v^{\lambda}\right)_{\lambda \in \Lambda}$ as $\lambda \downarrow 0$ is an immediate consequence of the linearity of $Y^{\nu}$ with respect to its initial condition.

Proposition 3.1 For all $\lambda \in \Lambda$ and $(t, x, p) \in[0, T] \times(0, \infty)^{d} \times[0,1]^{\kappa}$,

$$
v^{\lambda}(t, x, p \oplus \lambda)+2 \max _{i \leq \kappa} \lambda^{i} \geq v(t, x, p) \geq v^{\lambda}(t, x, p \ominus \lambda)-\max _{i \leq \kappa} \lambda^{i},
$$


where

$$
p \oplus \lambda:=\left(\left(\left(p^{i}+\lambda^{i}\right) \wedge 1\right) \vee 0\right)_{i \leq \kappa} \text { and } p \ominus \lambda:=\left(\left(\left(p^{i}-\lambda^{i}\right) \wedge 1\right) \vee 0\right)_{i \leq \kappa} .
$$

Proof. This follows easily from the linearity of $Y^{\nu}$ with respect to its initial condition and the fact that

$$
1_{\left\{y-g^{i}(x)+2 \lambda^{i} \geq 0\right\}}+\lambda^{i} \geq \Delta_{\lambda}^{i}(x, y) \geq 1_{\left\{y-g^{i}(x)+\lambda^{i} \geq 0\right\}}-\lambda^{i}, \text { for }(y, x) \in \mathbb{R} \times(0, \infty)^{d} .
$$

As an immediate consequence, we deduce that the sequences $\left(v^{\lambda}(\cdot, \cdot \oplus \lambda)\right)_{\lambda \in \Lambda}$ and $\left(v^{\lambda}(\cdot, \cdot \ominus \lambda)\right)_{\lambda \in \Lambda}$ allows to approximate $v$ at any continuity points in its $p$-variable. More precisely, the following holds.

Corollary 3.1 For all $(t, x, p) \in[0, T] \times(0, \infty)^{d} \times[0,1]^{\kappa}$,

$$
v(t, x, p-)=\liminf _{\lambda \downarrow 0} v^{\lambda}(t, x, p \ominus \lambda) \text { and } v(t, x, p+)=\limsup _{\lambda \downarrow 0} v^{\lambda}(t, x, p \oplus \lambda),
$$

where

$$
v(\cdot, p-):=\lim _{\varepsilon \downarrow 0} v\left(\cdot, p \ominus \varepsilon 1_{\kappa}\right) \quad \text { and } \quad v(\cdot, p+):=\lim _{\varepsilon \downarrow 0} v\left(\cdot, p \oplus \varepsilon 1_{\kappa}\right)
$$

with $1_{\kappa}=(1, \ldots, 1) \in \mathbb{R}^{\kappa}$.

Proving the continuity in its $p$-variable of the initial value function $v$ by probabilistic arguments, and therefore the point-wise convergence of our approximation seems very difficult, and is beyond the scope of this paper. A standard approach could be to derive the continuity of $v$ by using its PDE characterization and by applying a suitable comparison theorem which would imply that $v_{*}=v^{*}$. As explained in Section 2.3 , this also does not seem to be feasible.

Note however that the right- and left-limits of $v$ in its $p$-variable have interpretations in terms of natural relaxed version of the original problem (2.9):

$\underline{v}(t, x, p):=\inf \left\{y: \forall \varepsilon>0 \exists \nu^{\varepsilon} \in \mathcal{U}\right.$ s.t. $\left.Y_{t, x, y}^{\nu^{\varepsilon}}(T) \geq \ell, \mathbb{P}\left[Y_{t, x, y}^{\nu^{\varepsilon}}(T)-g\left(X_{t, x}(T)\right) \geq-\gamma^{i}\right] \geq p^{i}-\varepsilon \forall i \leq \kappa\right\}$, and

$$
\bar{v}(t, x, p):=\inf \left\{y: \exists \nu \in \mathcal{U} \text { s.t. } Y_{t, x, y}^{\nu}(T) \geq \ell, \mathbb{P}\left[Y_{t, x, y}^{\nu}(T)-g\left(X_{t, x}(T)\right) \geq-\gamma^{i}\right]>p^{i} \forall i \leq \kappa\right\} .
$$

Proposition 3.2 For all $(t, x, p) \in[0, T] \times(0, \infty)^{d} \times(0,1)^{\kappa}$,

$$
v(t, x, p+)=\bar{v}(t, x, p) \geq \underline{v}(t, x, p)=v(t, x, p-) .
$$

Proof. It is obvious that $\bar{v} \geq v \geq \underline{v}$. Moreover, any $y>v\left(t, x, p+\varepsilon 1_{\kappa}\right)$ for some $\varepsilon>0$, satisfies $y \geq \bar{v}(t, x, p)$. Hence, for $\varepsilon>0$ small enough, $v\left(t, x, p+\varepsilon 1_{\kappa}\right) \geq \bar{v}(t, x, p)$, so that $v(t, x, p+) \geq \bar{v}(t, x, p)$. Similarly, $y>\underline{v}(t, x, p)$ implies $y \geq v\left(t, x, p-\varepsilon 1_{\kappa}\right)$, for any $\varepsilon>0$ small enough, and therefore $\underline{v}(t, x, p) \geq$ $v(t, x, p-)$.

\subsection{PDE characterization of the approximating problems}

The reason for introducing the sequence approximating problems $\left(v^{\lambda}\right)_{\lambda \in \Lambda}$ is that they are more regular:

1. $\Delta_{\lambda}$ is Lipschitz continuous:

$$
\left|\Delta_{\lambda}^{i}(x, y+h)-\Delta_{\lambda}^{i}(x, y)\right| \leq C_{\lambda}|h|, \text { for }(x, y, h) \in(0, \infty)^{d} \times \mathbb{R} \times \mathbb{R} .
$$

where

$$
C_{\lambda}:=\max _{i \in \mathcal{K}} \max \left\{\lambda^{i} /\left(L^{-1}-2 \lambda^{i}\right), 1 / \lambda^{i}, 1\right\},
$$

recall (2.8) and (2.11). 
2. Its inverse with respect to its $y$-variable is Lipschitz continuous too. Hence, the natural boundary condition at $T$ is given by a continuous function

$$
G^{\lambda}(x, p):=\inf \left\{y \geq \ell: \min _{i \in \mathcal{K}}\left(\Delta_{\lambda}^{i}(x, y)-p^{i}\right) \geq 0\right\}, \text { for }(x, p) \in(0, \infty)^{d} \times[0,1]^{\kappa} .
$$

Item 2. above will allow us to prove that the boundary condition as $t \rightarrow T$ is indeed given by the continuous function $G^{\lambda}$, compare with 1. of Remark 2.13 .

Proposition 3.3 The function $v^{\lambda}$ satisfies

$$
v^{\lambda *}(T, \cdot)=v_{*}^{\lambda}(T, \cdot)=G^{\lambda} \text { on }(0, \infty)^{d} \times[0,1]^{\kappa} .
$$

Proof. See Section 4 below.

Item 1. above induces a gradient constraint on $v^{\lambda}$ with respect its $p$-variable, showing that it is strictly increasing with respect to this variable, in a suitable sense, which will allow us to prove a comparison result for the related PDE, compare with Remark 2.11 and 3. of Remark 2.13. We could not obtain this for the original problem by lack of continuity and local strict monotonicity of the indicator function. More precisely, we shall prove in Section 4.2 below the following.

Proposition 3.4 Set

$$
\varrho:=4 L^{2}(T \vee 1) .
$$

Fix $(I, J) \in \mathcal{P}_{\kappa} \backslash \mathcal{P}_{\kappa}^{\kappa}$ and assume that $\iota \geq 0$ is such that $v_{I J}^{\lambda *}(t, x, p)>v_{J^{c} J}^{\lambda *}(t, x, p)+\iota$. Let $\varphi$ be a smooth function such that $(t, x, p)$ achieves a maximum of $v_{I J}^{\lambda *}-\varphi$. Then,

$$
\sum_{i \notin I \cup J} D_{p^{i}} \varphi \geq \frac{\iota}{C_{\lambda}(\iota+\varrho)}=: \varpi_{\lambda}(\iota)
$$

where $C_{\lambda}$ is defined as in (3.5).

Note that the above can be translated in terms of the operator $M_{I J}^{\lambda}$ defined as:

$$
\left(y, z, q_{p}\right) \in \mathbb{R} \times \mathbb{R} \times \mathbb{R}^{\kappa} \mapsto M_{I J}^{\lambda}\left(y, z, q_{p}\right):=\max _{\iota \geq 0} \min \left\{y-z-\iota, \varpi_{\lambda}(\iota)-\sum_{i \notin I \cup J} q_{p}^{i}\right\} .
$$

Corollary 3.2 Fix $(I, J) \in \mathcal{P}_{\kappa} \backslash \mathcal{P}_{\kappa}^{\kappa}$. Then $v_{I J}^{\lambda *}$ is a viscosity subsolution on $D_{I J}$ of

$$
M_{I J}^{\lambda}\left(\varphi, v_{J^{c} J}^{\lambda *}, D_{p} \varphi\right)=0 .
$$

In view of Theorem 2.1 in [6], this implies that $v^{\lambda}$ is a discontinuous viscosity solution of the system $\left(\mathrm{S}^{\lambda}\right)$ defined as follows, where we use the convention

$$
M_{I J}^{\lambda}=-\infty \text { for } I \cup J=\mathcal{K} .
$$

Definition 3.1 Let $V$ be a locally bounded map defined on $\bar{D}$.

(i) We say that $V$ is a discontinuous viscosity supersolution of $\left(\mathrm{S}^{\lambda}\right)$ if, for each $(I, J) \in \mathcal{P}_{\kappa}, V_{I J *}$ is a viscosity supersolution on $D_{I J}$ of

$$
H_{I J}^{\lambda *}\left[\varphi, V_{J^{c} J^{*}}\right]:=\max \left\{\min \left\{\varphi-\ell,-\partial_{t} \varphi+F_{I J}^{*}\left(\cdot, D \varphi, D^{2} \varphi\right)\right\}, M_{I J}^{\lambda}\left(\varphi, V_{J^{c} J_{*}}, D_{p} \varphi\right)\right\}=0 .
$$

(ii) We say that $V$ is a discontinuous viscosity subsolution of $\left(\mathrm{S}^{\lambda}\right)$ if, for each $(I, J) \in \mathcal{P}_{\kappa}, V_{I J}^{*}$ is a viscosity subsolution on $D_{I J}$ of

$$
H_{I J *}^{\lambda}\left[\varphi, V_{J^{c} J}^{*}\right]:=\max \left\{\min \left\{\varphi-\ell,-\partial_{t} \varphi+F_{I J *}\left(\cdot, D \varphi, D^{2} \varphi\right)\right\}, M_{I J}^{\lambda}\left(\varphi, V_{J^{c} J}^{*}, D_{p} \varphi\right)\right\}=0 .
$$

(iv) We say that $V$ is a discontinuous viscosity solution of $\left(\mathrm{S}^{\lambda}\right)$ if it is both a discontinuous super-and subsolution of $\left(\mathrm{S}^{\lambda}\right)$. 
Remark 3.1 The convention (3.10) means that a supersolution of (3.11) (resp. a subsolution of (3.12)) for $I \cup J=\mathcal{K}$ is indeed a supersolution of (2.20) (resp. a subsolution of (2.21)).

Remark 3.2 Note that a viscosity supersolution of (2.20) on $D_{I J}$ is also a viscosity supersolution of (3.11) on $D_{I J}$. As already argued,

$$
v^{\lambda} \text { is a discontinuous solution of }(\mathrm{S})
$$

by Theorem 2.1 in [6], so that Corollary 3.2 implies that it is a discontinuous solution of $\left(\mathrm{S}^{\lambda}\right)$. From the supersolution point of view, the latter characterization is weaker. Still we shall use it because, first, it is sufficient and, second, we shall appeal to it when discussing the convergence of a finite difference approximation scheme below.

Combining the above results, we obtain:

Theorem 3.1 The function $v^{\lambda}$ is a discontinuous viscosity solution of $\left(\mathrm{S}^{\lambda}\right)$. Moreover, it satisfies

$$
v^{\lambda *}(T, \cdot)=v_{*}^{\lambda}(T, \cdot)=G^{\lambda} \text { on }(0, \infty)^{d} \times[0,1]^{\kappa} .
$$

The fact that the above Theorem allows to characterize uniquely $v^{\lambda}$ is a consequence of the following comparison result, in the viscosity sense.

Theorem 3.2 (i) Let $V$ be a bounded function on $[0, T) \times(0, \infty)^{d} \times[0,1]^{\kappa}$ which is non-decreasing with respect to its last parameter. Assume that $V$ is a discontinuous viscosity supersolution of $\left(\mathrm{S}^{\lambda}\right)$ such that $V_{*}(T, \cdot) \geq G^{\lambda}$ and $V_{I J *} \geq V_{I^{\prime} J^{\prime} *}$ on $\partial D_{I J} \cap D_{I^{\prime} J^{\prime}}$ for all $(I, J),\left(I^{\prime}, J^{\prime}\right) \in \mathcal{P}_{\kappa}$ such that $\left(I^{\prime}, J^{\prime}\right) \supsetneq(I, J)$. Then, $V \geq v^{\lambda}$ on $\bar{D}$.

(ii) Let $V$ be a bounded function on $[0, T) \times(0, \infty)^{d} \times[0,1]^{\kappa}$ which is non-decreasing with respect to its last parameter. Assume that $V$ is a discontinuous viscosity subsolution of $\left(\mathrm{S}^{\lambda}\right)$ such that $V^{*}(T, \cdot) \leq G^{\lambda}$ and $V_{I J}^{*} \leq V_{I^{\prime} J^{\prime}}^{*}$ on $\partial D_{I J} \cap D_{I^{\prime} J^{\prime}}$ for all $(I, J),\left(I^{\prime}, J^{\prime}\right) \in \mathcal{P}_{\kappa}$ such that $\left(I^{\prime}, J^{\prime}\right) \supsetneq(I, J)$. Then, $V \leq v^{\lambda}$ on $\bar{D}$.

Proof. See Section 4.4 below.

Combining the above results leads to the following characterization.

Theorem 3.3 The function $v^{\lambda}$ is continuous and is the unique bounded discontinuous viscosity solution of the system $\left(\mathrm{S}^{\lambda}\right)$ in the class of bounded discontinuous solutions $V$ which are non-decreasing in their last variable and satisfy $V_{*}(T, \cdot)=V^{*}(T, \cdot)=G^{\lambda}, V_{I J}^{*} \leq V_{I^{\prime} J^{\prime}}^{*}$ and $V_{I J *} \geq V_{I^{\prime} J^{\prime} *}$ on $\partial D_{I J} \cap D_{I^{\prime} J^{\prime}}$ for all $(I, J),\left(I^{\prime}, J^{\prime}\right) \in \mathcal{P}_{\kappa}$ such that $\left(I^{\prime}, J^{\prime}\right) \supsetneq(I, J)$.

\subsection{Finite differences approximation}

In this section, we construct an explicit finite difference scheme and prove its convergence.

\subsubsection{PDE reformulation}

We first reformulate the PDEs associated to $v^{\lambda}$ in a more tractable way, which will allow us to define naturally a monotone scheme. To this purpose, we introduce the support function $\delta_{U}$ associated to the closed convex (and bounded) set $U$ as in (2.24). Since $0 \in \operatorname{int} U, \delta_{U}$ characterizes $U$ in the following sense

$$
u \in \operatorname{int} U \text { iff } \min _{|\zeta|=1}\left(\delta_{U}(\zeta)-\zeta^{\top} u\right)>0 \text { and } u \in U \text { iff } \min _{|\zeta|=1}\left(\delta_{U}(\zeta)-\zeta^{\top} u\right) \geq 0
$$


see e.g. [13].

Moreover $(u, a) \in N_{I J}^{\epsilon}(x, q)$ with $q^{\top}=\left(q_{x}^{\top}, q_{p}^{\top}\right)$, if and only if there exists $\xi_{\epsilon} \in \mathbb{R}^{d}$ such that $\left|\xi_{\epsilon}\right| \leq \epsilon$ for which

$$
u^{\top}=\bar{u}(x, a, q)^{\top}+\xi_{\epsilon} \sigma(x)^{-1} \in U \text { and } a \in A_{I J},
$$

where

$$
\bar{u}(x, q, a)^{\top}:=q_{x}^{\top} \operatorname{diag}[x]+q_{p}^{\top} a \sigma(x)^{-1} .
$$

It follows that

$$
-r+F_{I J}^{*}(x, q, Q) \geq 0 \text { iff } \bar{K}_{I J}^{*}(x, r, q, Q) \geq 0 \text { and }-r+F_{I J *}(x, q, Q) \leq 0 \text { iff } \bar{K}_{I J *}(x, r, q, Q) \leq 0
$$

where $\bar{K}_{I J}^{*}$ and $\bar{K}_{I J *}$ are the upper- and lower-semicontinuous envelopes of

$$
\bar{K}_{I J}(x, r, q, Q):=\sup _{a \in A_{I J}} \min \left\{-r+L^{\bar{u}(x, q, a), a}(x, q, Q), R^{a}(x, q)\right\}
$$

with

$$
R^{a}(x, q):=\inf _{|\zeta|=1} R^{a, \zeta}(x, q) \text { and } R^{a, \zeta}(x, q):=\delta_{U}(\zeta)-\zeta^{\top} \bar{u}(x, q, a) .
$$

Remark 3.3 For later use, note that, for $q^{\top}=\left(q_{x}^{\top}, q_{p}^{\top}\right)$,

$$
L^{\bar{u}(x, q, a), a}(x, q, Q)=q_{p}^{\top} a \sigma(x)^{-1} \mu(x)-\frac{1}{2} \Xi^{a}(x, Q)=: \bar{L}^{a}\left(x, q_{p}, \Xi^{a}(x, Q)\right) .
$$

where

$$
\Xi^{a}(x, Q):=\operatorname{Trace}\left[\sigma_{X, P} \sigma_{X, P}^{\top}(x, a) Q\right]
$$

does not depend on $q_{x}$.

It follows that $V$ is a viscosity supersolution of (3.11) if and only if it is a viscosity supersolution of

$$
\bar{H}_{I J}^{\lambda *}\left[\varphi, V_{J^{c} J *}\right]:=\max \left\{\min \left\{\varphi-\ell, \bar{K}_{I J}^{*}\left(\cdot, \partial_{t} \varphi, D \varphi, D^{2} \varphi\right)\right\}, M_{I J}^{\lambda}\left(\varphi, V_{J^{c} J *}, D_{p} \varphi\right)\right\}=0,
$$

and that $V$ is a viscosity subsolution of (3.12) if and only if it is a viscosity subsolution of

$$
\bar{H}_{I J *}^{\lambda}\left[\varphi, V_{J^{c} J}^{*}\right]:=\max \left\{\min \left\{\varphi-\ell, \bar{K}_{I J *}\left(\cdot, \partial_{t} \varphi, D \varphi, D^{2} \varphi\right)\right\}, M_{I J}^{\lambda}\left(\varphi, V_{J^{c} J}^{*}, D_{p} \varphi\right)\right\}=0 .
$$

\subsubsection{Scheme construction}

We now define a monotone finite difference scheme for the formulation obtained in the previous section. In the following, we write $h$ to denote an element of the form $h=\left(h_{0}, h_{1}, h_{2}\right) \in(0,1)^{3}$.

a. The discretization in the time variable.

Given $n_{0} \in \mathbb{N}$, we first introduce a discretization time-step $h_{0}:=T / n_{0}$ together with a grid

$$
\mathbf{T}^{h}:=\left\{\left(T-\left(n_{0}-i\right) h_{0}\right), i=0, \ldots, n_{0}\right\} .
$$

The time derivative is approximated as usual by

$$
\partial_{t}^{h}[\varphi](t, x, p):=h_{0}^{-1}\left(\varphi\left(t+h_{0}, x, p\right)-\varphi(t, x, p)\right) .
$$

\section{b. The discretization in the space variable.}

The grids in the space variables are defined as

$$
\mathbf{X}_{c_{X}}^{h}:=\left\{e^{-c_{X}+(n-i) h_{1}}, i=0, \ldots, n_{X}\right\}^{d} \text { and } \mathbf{P}^{h}:=\left\{1-(n-i) h_{1}, i=0, \ldots, n_{P}\right\}^{\kappa},
$$


for some $c_{X}, n \in \mathbb{N}$ and where $h_{1}:=1 / n,\left(n_{X}, n_{P}\right):=n\left(2 c_{X}, 1\right)$.

Note that the space discretization in the $x$ variable amounts to performing a logarithmic change of variable. Taking this into account, the first order derivatives with respect to $x$ and $p$ are then approximated as follows, with $\left\{e_{i}\right\}_{i \leq d}$ (resp. $\left.\left\{\ell_{j}\right\}_{j \leq \kappa}\right)$ denoting the canonical basis of $\mathbb{R}^{d}$ (resp. $\mathbb{R}^{\kappa}$ ):

$$
\begin{aligned}
\partial_{p, j}^{L, h}[\varphi, a](t, x, p) & :=h_{1}^{-1} \begin{cases}\varphi\left(t+h_{0}, x, p \oplus h_{1} \ell_{j}\right)-\varphi(t, x, p) & \text { if } \quad \mu^{\top}\left(a \sigma^{-1}(x)\right)^{\top} \ell_{j} \leq 0 \\
\varphi(t, x, p)-\varphi\left(t+h_{0}, x, p \ominus h_{1} \ell_{j}\right) & \text { if } \quad \mu^{\top}\left(a \sigma^{-1}(x)\right)^{\top} \ell_{j}>0\end{cases} \\
\partial_{x, i}^{R, h}[\varphi, \zeta](t, x, p) & :=h_{1}^{-1} \operatorname{diag}[x]^{-1} \begin{cases}\varphi\left(t+h_{0}, x \widehat{\oplus} h_{1} e_{i}, p\right)-\varphi(t, x, p) & \text { if } \quad e_{i}^{\top} \zeta \geq 0 \\
\varphi(t, x, p)-\varphi\left(t+h_{0}, x \widehat{\ominus} h_{1} e_{i}, p\right) & \text { if } \quad e_{i}^{\top} \zeta<0\end{cases} \\
\partial_{p, j}^{R, h}[\varphi, a, \zeta](t, x, p) & :=h_{1}^{-1} \begin{cases}\varphi\left(t+h_{0}, x, p \oplus h_{1} \ell_{j}\right)-\varphi(t, x, p) & \text { if } \quad \ell_{j}^{\top} a \sigma^{-1}(x) \zeta \geq 0 \\
\varphi(t, x, p)-\varphi\left(t+h_{0}, x, p \ominus h_{1} \ell_{j}\right) & \text { if } \quad \ell_{j}^{\top} a \sigma^{-1}(x) \zeta<0\end{cases} \\
\partial_{p, j}^{M, h}[\varphi](t, x, p) & :=h_{1}^{-1}\left(\varphi\left(t+h_{0}, x, p \oplus h_{1} \ell_{j}\right)-\varphi(t, x, p)\right),
\end{aligned}
$$

where the operators $\oplus$ and $\ominus$ are given in Proposition 3.1 and

$\left.x \widehat{\oplus} y:=\left(\left(x^{i} e^{y^{i}}\right) \vee e^{-c_{X}}\right) \wedge e^{c_{X}}\right)_{i \leq d}$ and $\left.x \widehat{\ominus} y:=\left(\left(x^{i} e^{-y^{i}}\right) \vee e^{-c_{X}}\right) \wedge e^{c_{X}}\right)_{i \leq d}, \quad$ for $(x, y) \in(0, \infty)^{d} \times \mathbb{R}^{d}$.

We denote by $\partial_{p}^{L, h}, \partial_{x}^{K, h}, \partial_{p}^{K, h}$ and $\partial_{p}^{M, h}$ the corresponding vectors.

As for the second order term, we use the Camilli and Falcone approximation [7], in order to ensure that the scheme is monotone. Namely, we first introduce an approximation parameterized by $h_{2}>0$ of Trace $\left[\sigma_{(X, P)} \sigma_{(X, P)}^{\top}(x, a) D^{2} \varphi\left(t+h_{0}, x, p\right)\right]$ as follows

$$
\begin{aligned}
& \tilde{\Delta}^{h}[\varphi, a](t, x, p) \\
& :=h_{2}^{-1} \sum_{i=1}^{d}\left(\varphi\left(t+h_{0}, x \widehat{\oplus} \sqrt{h_{2}} \sigma^{\cdot i}(x), p \oplus \sqrt{h_{2}} a^{\cdot i}\right)+\varphi\left(t+h_{0}, x \widehat{\ominus} \sqrt{h_{2}} \sigma^{\cdot i}(x), p \ominus \sqrt{h_{2}} a^{\cdot i}\right)-2 \varphi\left(t+h_{0}, x, p\right)\right) \\
& -h_{1}^{-1} \sum_{i=1}^{d}\left|\sigma^{\cdot i}(x)\right|^{2}\left(\varphi\left(t_{0}, x, p\right)-\varphi\left(t+h_{0}, x \widehat{\ominus} h_{1} e_{i}, p\right)\right)
\end{aligned}
$$

where $\sigma^{\cdot i}$ and $a^{\cdot i}$ denote the $i$-th column of $\sigma_{X}$ and $a$.

Note that the above approximation of the second order term requires the computation of the approximated value function at points outside of the grid. It therefore requires an interpolation procedure. In this paper, we use a local linear interpolation based on the Coxeter-Freudenthal-Kuhn triangulation, see e.g. [12]. It consists in first constructing the set of simplices $\left\{S_{j}\right\}_{j}$ associated to the regular triangulation of $\ln \left[e^{-c_{X}}, e^{c_{X}}\right]^{d} \times[0,1]^{\kappa}$ with the set of vertices $\ln \mathbf{X}_{c_{X}}^{h} \times \mathbf{P}^{h}$. Here the $\ln$ operator means that we take the ln component-wise, recall that we use a logarithmic scale. In such a way, we can then provide an approximating function belonging to the set $\mathcal{S}^{h}$ of the functions which are continuous in $\left[e^{-c_{X}}, e^{c_{X}}\right]^{d} \times[0,1]^{\kappa}$ and piecewise affine inside each simplex $S_{j}$ (in ln scale for the $x$-variable). More precisely, each point $(y, p) \in\left[-c_{X}, c_{X}\right]^{d} \times[0,1]^{\kappa}$ can be expressed as a weighted combination of the corners of the simplex $S_{j}$ it lies in. We can thus write

$$
(y, p)=\sum_{\zeta \in \ln \mathbf{X}_{c_{X}}^{h} \times \mathbf{P}^{h}} \omega(y, p \mid \zeta) \zeta
$$

where $\omega$ is a non negative weighting function such that

$$
\sum_{\zeta \in \ln \mathbf{X}_{c_{X}}^{h} \times \mathbf{P}^{h}} \omega(y, p \mid \zeta)=1 .
$$


Given a map $\varphi$ defined on $\mathbf{T}^{h} \times \mathbf{X}_{c_{X}}^{h} \times \mathbf{P}^{h}$, we then approximate it at $(t, x, p) \in \mathbf{T}^{h} \times\left[e^{-c_{X}}, e^{c_{X}}\right]^{d} \times[0,1]^{\kappa}$ by

$$
\varphi(t, x, p):=\sum_{\left(\zeta_{X}, \zeta_{P}\right) \in \ln \mathbf{X}_{c_{X}}^{h} \times \mathbf{P}^{h}} \omega(\ln x, p \mid \zeta) \varphi\left(t, e^{\zeta X}, \zeta_{P}\right)
$$

in which the exponential is taken component by component.

This leads to the approximation of $\tilde{\Delta}^{h}[\varphi, a](t, x, p)$ by

$$
\begin{aligned}
\Delta^{h}[\varphi, a](t, x, p) \quad & :=h_{2}^{-1} \sum_{i=1}^{d} \sum_{\left(\zeta_{X}, \zeta_{P}\right) \in \ln \mathbf{X}_{c_{X}}^{h} \times \mathbf{P}^{h}} \omega\left(x_{h+}^{i}, p_{h+}^{i}[a] \mid\left(\zeta_{X}, \zeta_{P}\right)\right) \varphi\left(t+h_{0}, e^{\zeta_{X}}, \zeta_{P}\right) \\
& +h_{2}^{-1} \sum_{i=1}^{d} \sum_{\left(\zeta_{X}, \zeta_{P}\right) \in \ln \mathbf{X}_{c_{X}}^{h} \times \mathbf{P}^{h}} \omega\left(x_{h-}^{i}, p_{h-}^{i}[a] \mid\left(\zeta_{X}, \zeta_{P}\right)\right) \varphi\left(t+h_{0}, e^{\zeta_{X}}, \zeta_{P}\right) \\
& -2 d h_{2}^{-1} \varphi\left(t+h_{0}, x, p\right) \\
& -h_{1}^{-1} \sum_{i=1}^{d}\left|\sigma^{\cdot i}(x)\right|^{2}\left(\varphi\left(t+h_{0}, x, p\right)-\varphi\left(t+h_{0}, x \widehat{\ominus} h_{1} e_{i}, p\right)\right)
\end{aligned}
$$

where

$$
x_{h+}^{i}:=x \widehat{\oplus} \sqrt{h_{2}} \sigma^{\cdot i}(x), p_{h+}^{i}[a]:=p \oplus \sqrt{h_{2}} a^{\cdot i} \text {, and } x_{h-}^{i}:=x \widehat{\ominus} \sqrt{h_{2}} \sigma^{\cdot i}(x), p_{h-}^{i}[a]:=p \ominus \sqrt{h_{2}} a^{\cdot i} .
$$

\section{c. The approximated operator.}

Given $\bar{a}>0$, we then approximate $\bar{H}_{I J}^{\lambda}$ by $\hat{H}_{I J}^{h, c_{X}, \bar{a}}$ defined as

$$
\hat{H}_{I J}^{h, c_{X}, \bar{a}}[\varphi, \psi]:=\max \left\{\min \left\{\varphi-\ell, \sup _{a \in A_{I J}^{\bar{a}}} \hat{K}_{h}^{a} \varphi\right\}, \hat{M}_{h}^{I J}[\varphi, \psi]\right\}
$$

with

$$
A_{I J}^{\bar{a}}:=\left\{a \in A_{I J}:|a| \leq \bar{a}\right\}, \hat{M}_{h}^{I J}[\varphi, \psi]:=M^{I J}\left(\varphi, \psi, \partial_{p}^{M, h}[\varphi]\right)
$$

and

$$
\hat{K}_{h}^{a} \varphi:=\min \left\{-\partial_{t}^{L, h} \varphi+\bar{L}^{a}\left(\cdot, \partial_{p}^{L, h} \varphi, \Delta^{L, h}[\varphi, a]\right), \min _{|\zeta|=1} R^{a, \zeta}\left(\cdot, \partial_{x}^{R, h}[\varphi, \zeta], \partial_{p}^{R, h}[\varphi, a, \zeta]\right)\right\} .
$$

The resolution is done as follows:

(i). For $(I, J) \in \mathcal{P}_{\kappa}^{\kappa}$, we define $w_{I J}^{\bar{a}, c_{X}, h} \in \mathcal{S}^{h}$ as the solution of

$$
\left\{\begin{array}{ccc}
w_{I J}^{\bar{a}, c_{X}, h}(T, \cdot)=G^{\lambda}\left(\cdot, \pi_{I J}\right) & \text { on } & \mathbf{X}_{c_{X}}^{h} \times \mathbf{P}^{h} \\
\max \left\{w_{I J}^{\bar{a}, c_{X}, h}-L, \hat{H}_{I J}^{h, c_{X}, \bar{a}}\left[w_{I J}^{\bar{a}, c_{X}, h}, 0\right]\right\}=0 & \text { on } & \mathbf{T}_{-}^{h} \times \mathbf{X}_{c_{X}-}^{h} \times \mathbf{P}^{h} \\
w_{I J}^{\bar{a}, c_{X}, h}=\hat{g}_{J} & \text { on } & \mathbf{T}_{-}^{h} \times\left(\mathbf{X}_{c_{X}}^{h} \backslash \mathbf{X}_{c_{X}-}^{h}\right) \times \mathbf{P}^{h}
\end{array} .\right.
$$

where we use the notations

$$
\mathbf{T}_{-}^{h}:=\left\{\left(T-\left(n_{0}-i\right) h_{0}\right), i=0, \ldots, n_{0}-1\right\} \text { and } \mathbf{X}_{c_{X}-}^{h}:=\left\{e^{-c_{X}+(n-i) h_{1}}, i=1, \ldots, n_{X}-1\right\}^{d} .
$$

(ii). We then proceed by backward induction on $|I|+|J|$. Once $w_{I^{\prime} J^{\prime}}^{\bar{a}, c_{X}, h} \in \mathcal{S}^{h}$ constructed for $\left(I^{\prime}, J^{\prime}\right) \in$ $\mathcal{P}_{\kappa}^{l}$ for all $l \geq k$, for some $1 \leq k \leq \kappa$, we define $w_{I J}^{\bar{a}, c_{X}, h}$ for $(I, J) \in \mathcal{P}_{\kappa}^{k-1}$ as the solution of

$$
\left\{\begin{array}{c}
w_{I J}^{\bar{a}, c_{X}, h}(T, \cdot)=G^{\lambda}\left(\cdot, \pi_{I J}\right) \text { on } \mathbf{X}_{c_{X}}^{h} \times \mathbf{P}^{h} \\
\left(w_{I J}^{\bar{a}, c_{X}, h}-w_{J^{c} J}^{\bar{a}, c_{X}, h}\right) \wedge \max \left\{w_{I J}^{\bar{a}, c_{X}, h}-L, \hat{H}_{I J}^{h, c_{X}, \bar{a}}\left[w_{I J}^{\bar{a}, c_{X}, h}, w_{J^{c} J}^{\bar{a}, c_{X}, h}\right]\right\}=0 \text { on }\left(\mathbf{T}_{-}^{h} \times \mathbf{X}_{c_{X}-}^{h} \times \mathbf{P}^{h}\right) \cap D_{I J} \\
w_{I J}^{\bar{a}, c_{X}, h}=G^{\lambda}\left(\cdot, \pi_{I J}\right) \text { on }\left(\mathbf{T}_{-}^{h} \times\left(\mathbf{X}_{c_{X}}^{h} \backslash \mathbf{X}_{c_{X}-}^{h}\right) \times \mathbf{P}^{h}\right) \cap D_{I J} \\
w_{I J}^{\bar{a}, c_{X}, h}=w_{I^{\prime} J^{\prime}}^{\bar{a}, c_{X}, h} \text { on }\left(\mathbf{T}_{-}^{h} \times \mathbf{X}_{c_{X}-}^{h} \times \mathbf{P}^{h}\right) \cap \partial D_{I J} \cap D_{I^{\prime} J^{\prime}} \text { for }\left(I^{\prime}, J^{\prime}\right) \supsetneq(I, J)
\end{array}\right.
$$


One easily checks that

$$
\left|\tilde{\Delta}^{h}[\varphi, a](t, x, p)-\Delta^{h}[\varphi, a](t, x, p)\right| \leq O\left(h_{1} / h_{2}\right),
$$

which implies that the numerical scheme is monotone and consistent whenever

$$
h_{0}=o\left(h_{1}\right) \text { and } h_{1}=o\left(h_{2}\right) \text {. }
$$

\subsubsection{Convergence of the approximating scheme}

The convergence of the scheme is obtained as $h=\left(h_{0}, h_{1}, h_{2}\right) \rightarrow 0$ and $c_{X} \rightarrow \infty$, with the convention (3.19), and then $\bar{a} \rightarrow \infty$. We therefore define the relaxed semi-limits, for $(t, x, p) \in \bar{D}_{I J},(I, J) \in \mathcal{P}_{\kappa}$,

$\bar{w}_{I J}^{\bar{a} *}(t, x, p):=\limsup _{\substack{\left(t^{\prime}, x^{\prime}, p^{\prime}\right) \rightarrow(t, x, p) \\ h \rightarrow 0, c_{X} \rightarrow \infty}} w_{I J}^{\bar{a}, c_{X}, h}\left(t^{\prime}, x^{\prime}, p^{\prime}\right), \bar{w}_{I J *}^{\bar{a}}(t, x, p):=\liminf _{\substack{\left(t^{\prime}, x^{\prime}, p^{\prime}\right) \rightarrow(t, x, p) \\ h \rightarrow 0, c_{X} \rightarrow \infty}} w_{I J}^{\bar{a}, c_{X}, h}\left(t^{\prime}, x^{\prime}, p^{\prime}\right)$

and

$$
\bar{w}_{I J}^{*}(t, x, p):=\limsup _{\substack{\left(t^{\prime}, x^{\prime}, p^{\prime}\right) \rightarrow(t, x, p) \\ \bar{a} \rightarrow \infty}} w_{I J}^{\bar{a} *}\left(t^{\prime}, x^{\prime}, p^{\prime}\right), \bar{w}_{I J *}(t, x, p):=\liminf _{\substack{\left(t^{\prime}, x^{\prime}, p^{\prime}\right) \rightarrow(t, x, p) \\ \bar{a} \rightarrow \infty}} w_{I J *}^{\bar{a}}\left(t^{\prime}, x^{\prime}, p^{\prime}\right),
$$

in which the limits are taken along sequences of points $\left(t^{\prime}, x^{\prime}, p^{\prime}\right) \in \bar{D}_{I J}$ and $h$ satisfying (3.19). Note that $w_{I J}^{\bar{a}, c_{X}, h}$ takes values in $[\ell, L]$, so that the above are well-defined and bounded. Moreover, it is convergent:

Theorem 3.4 For all $(I, J) \in \mathcal{P}_{\kappa}, \bar{w}_{I J}^{*}=\bar{w}_{I J *}=v_{I J}^{\lambda}$ on $\bar{D}_{I J}$.

Proof. See Section 4.5 below.

We conclude this section with some numerical illustration in the Black and Scholes model, where the stock price $X$ is defined as

$$
X_{t, x}(s)=x+\int_{t}^{s} X_{t, x}(r) d W_{r} \text { for } s \in[t, 1]
$$

the payoff $g(X)=(K-X)_{+}$with the strike price $K=3$, the thresholds $\gamma=\left\{\gamma^{1}, \gamma^{2}\right\}=\{0,0.5\}$.

Example 3.1 We study the case $U=[-1,1]$.

Then, the "face-lifted" version of $g$ is defined by

$$
\hat{g}(x)=\left\{\begin{array}{ccc}
3-x & \text { if } & x \in(0,1] \\
2-\ln (x) & \text { if } & x \in\left[1, e^{2}\right] . \\
0 & \text { if } & x \geq e^{2}
\end{array}\right.
$$

Taking $\lambda=1 / 32$ and $\ell=-1$, the Figure 2 plots an estimated value of $v^{\lambda}\left(0, x, p^{1}, p^{2}\right)$ when we fix $x=e$.

Example 3.2 When $U=[-5,5]$, the "face-lifted" version of $g$ is equal to $g$ on $\mathbb{R}_{+}$. The Figure 3 plots an estimated value of $v^{\lambda}\left(0, x, p^{1}, p^{2}\right)$ when we take $\lambda=1 / 32, \ell=-1$ and $x=1$. In the Figure 4 , we describe its graph when $p^{2}=0$ in the same setting. 


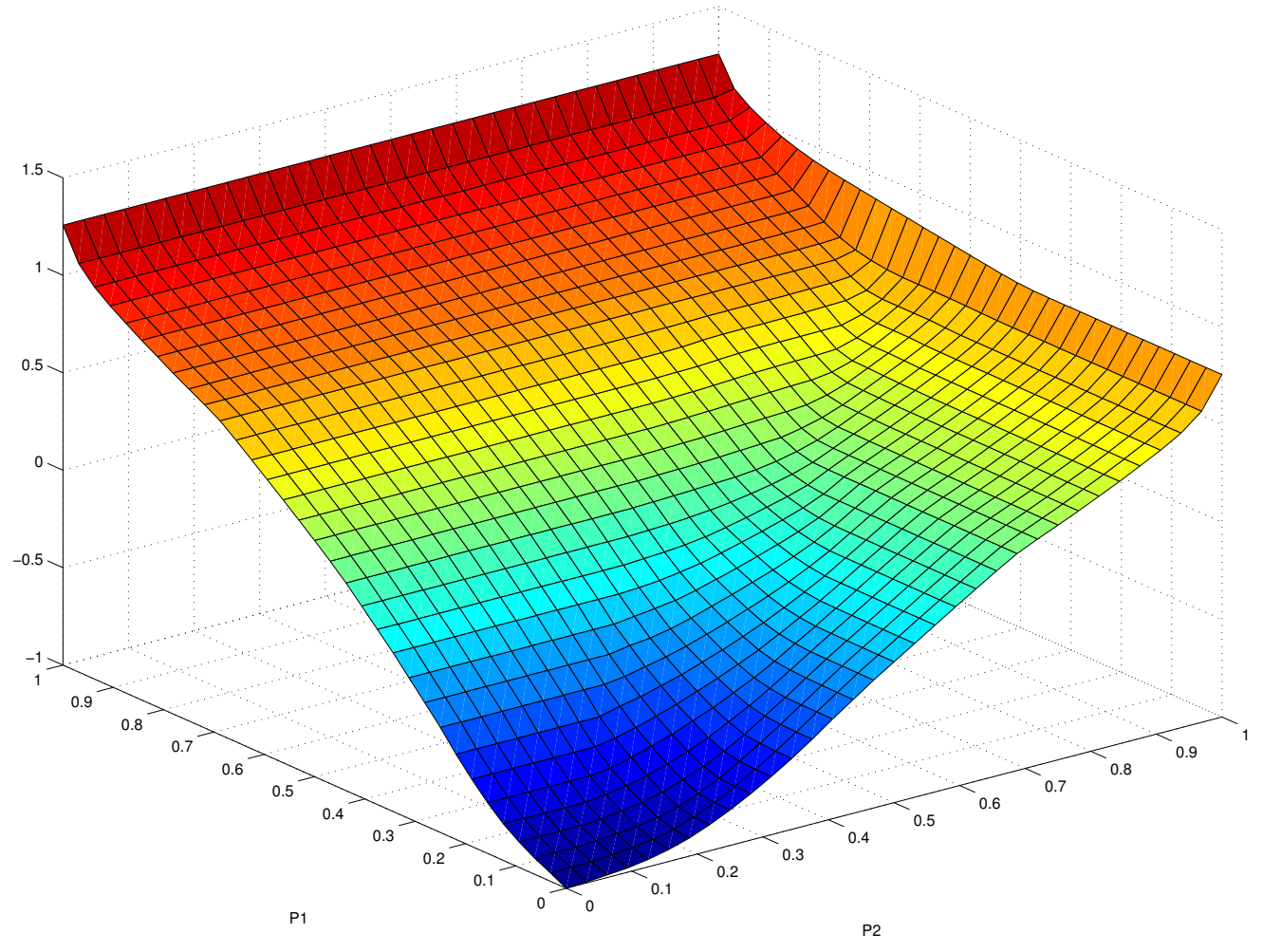

Figure 2: $v^{\lambda}$ with $U=[-1,1]$

\section{Proof of the PDE characterizations and of the convergence result}

In this section, we collect the proofs of Proposition 3.3, Proposition 3.4, Theorem 3.2, Theorem 3.3 and Theorem 3.4. We start with the boundary conditions in time and in the space variable $p$. We first recall the geometric dynamic programming principle of [14], see also [15] and [16], to which we will appeal to prove the boundary conditions. We next report the proof of the supersolution properties in subsection 4.1.2, and that of the subsolution properties in subsection 4.1.1. The gradient estimates in the viscosity sense and the corresponding comparison result are proved in next subsection.

\subsection{Boundary conditions}

In the following, $\mathcal{T}_{[s, t]}$ denotes the set of $[s, t]$-valued stopping times.

Corollary 4.1 Fix $(t, x, p) \in \bar{D}_{I J}$.

(GDP1) If $y \geq \ell$ and $(\nu, \alpha) \in \mathcal{U} \times \mathcal{A}_{I J}$ are such that $\Delta_{\lambda}\left(X_{t, x},(T), Y_{t, x, y}^{\nu}(T)\right) \geq P_{t, p}^{\alpha}(T)$ then

$$
Y_{t, x, y}^{\nu}(\theta) \geq v_{I J}^{\lambda}\left(\theta, X_{t, x}(\theta), P_{t, p}^{\alpha}(\theta)\right), \quad \text { for all } \theta \in \mathcal{T}_{[t, T]} .
$$




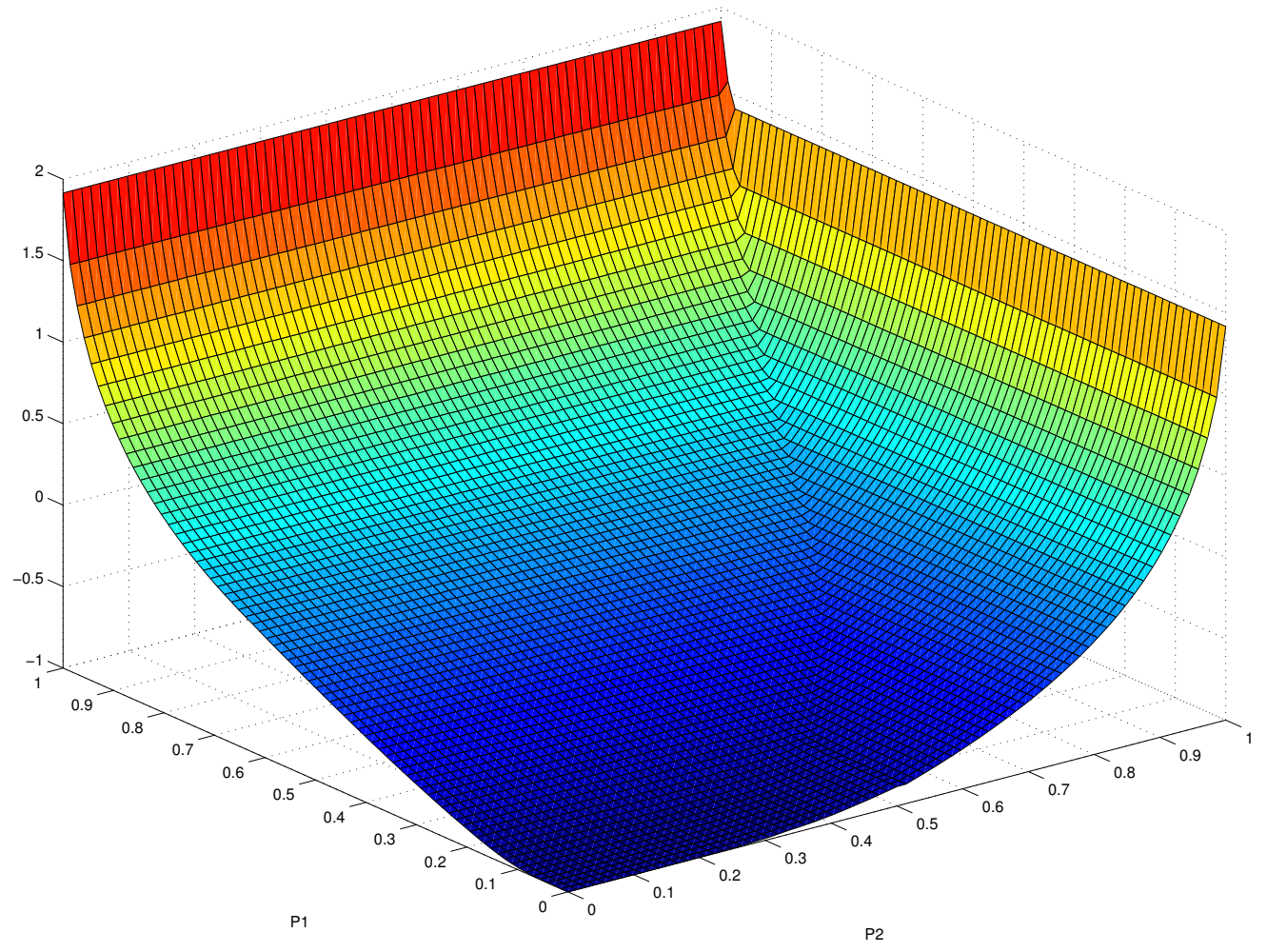

Figure 3: $v^{\lambda}$ with $U=[-5,5]$

(GDP2) For $y<v_{I J}^{\lambda}(t, x, p), \theta \in \mathcal{T}_{[t, T]}$ and $(\nu, \alpha) \in \mathcal{U} \times \mathcal{A}$,

$$
\mathbb{P}\left[Y_{t, x, y}^{\nu}(\theta)>v_{I J}^{\lambda}\left(\theta, X_{t, x}(\theta), P_{t, p}^{\alpha}(\theta)\right)\right]<1 .
$$

\subsubsection{Boundary condition for the upper-semicontinuous enveloppe}

We start with the boundary condition as $t \rightarrow T$.

Proposition 4.1 For all $(I, J),\left(I^{\prime}, J^{\prime}\right) \in \mathcal{P}_{\kappa}$ such that $\left(I^{\prime}, J^{\prime}\right) \supset(I, J)$, we have

$$
v_{I J}^{\lambda *}(T, \cdot) \leq G^{\lambda} \text { on }(0, \infty)^{d} \times \bar{B}_{I^{\prime} J^{\prime}} .
$$

Proof.

Step 1. We first show that the required result is true if $I \cup J=\mathcal{K}$. Note that, in this case, $I^{\prime}=I$ and $J^{\prime}=J$. Then,

$$
v_{I J}^{\lambda}=w:=\inf \left\{y \geq \ell: \exists \nu \in \mathcal{U} \text { s.t. } Y_{\cdot, y}^{\nu}(T) \geq \hat{g}_{J}(X .(T))\right\} .
$$

Hence, it suffices to show that

$$
w^{*}(T, \cdot) \leq \hat{g}_{J}
$$

where $w^{*}(T, x):=\lim _{\varepsilon \rightarrow 0} \sup \left\{w\left(t^{\prime}, x^{\prime}\right):\left(t^{\prime}, x^{\prime}\right) \in(T-\varepsilon, T] \times B_{\varepsilon}\left(x^{\prime}\right)\right\}$. We only sketch the proof of (4.1) as it follows from the same arguments as in [3], up to obvious modifications. In the following, we let $\left(t_{n}, x_{n}\right)_{n}$ be a sequence in $[0, T) \times(0, \infty)^{d}$ such that $\left(t_{n}, x_{n}\right) \rightarrow(T, x)$ and $w\left(t_{n}, x_{n}\right) \rightarrow w^{*}(T, x)$. 


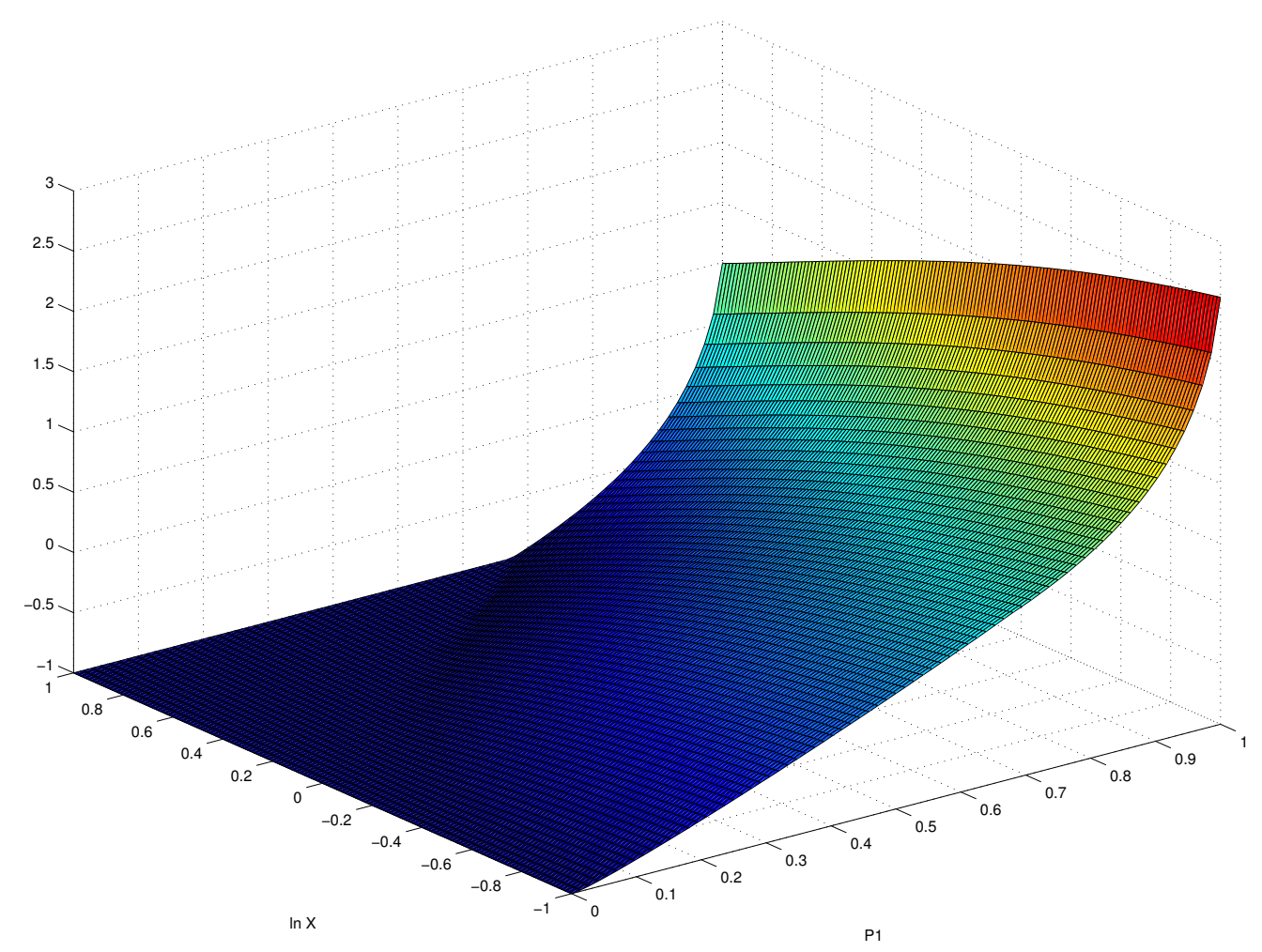

Figure 4: $\mathrm{U}=[-5,5]$ and $p^{2}=0$

It follows from the dual formulation of [10] that, for each $n \geq 1$, we can find a predictable process $\vartheta^{n}$ with values in $\mathbb{R}^{d}$ such that

$$
H_{n}^{\vartheta^{n}}:=\mathcal{E}\left(-\int_{t_{n}}^{\cdot} \sigma_{X}^{-1}\left(\mu_{X}-\vartheta_{s}^{n}\right)\left(X_{n}(s)\right) d W_{s}\right)_{T} \in L^{1}(\mathbb{P}),
$$

where $X_{n}:=X_{t_{n}, x_{n}}$, and

$$
w\left(t_{n}, x_{n}\right) \leq \mathbb{E}\left[H_{n}^{\vartheta^{n}}(T)\left(g_{J}\left(X_{n}(T)\right)-\int_{t_{n}}^{T} \delta_{U}\left(\vartheta_{s}^{n}\right) d s\right)\right]+n^{-1} .
$$

Since $\delta_{U}$ is homogeneous of degree 1 and convex, this implies that

$$
w\left(t_{n}, x_{n}\right) \leq \mathbb{E}\left[H_{n}^{\vartheta^{n}}(T)\left(g_{J}\left(X_{n}(T)\right)-\delta_{U}\left(\int_{t_{n}}^{T} \vartheta_{s}^{n}\right) d s\right)\right]+n^{-1}
$$

so that, by definition of $\hat{g}_{J}$ in (2.23),

$$
w\left(t_{n}, x_{n}\right) \leq \mathbb{E}\left[H_{n}^{\vartheta^{n}}(T) \hat{g}_{J}\left(Z_{n}^{\vartheta^{n}}(T)\right)\right]+n^{-1},
$$

where $Z_{n}:=X_{n} e^{-\int_{t_{n}} \vartheta_{s}^{n} d s}$. It remains to prove that

$$
\limsup _{n \rightarrow \infty} \mathbb{E}\left[H_{n}^{\vartheta^{n}}(T) \hat{g}_{J}\left(Z_{n}^{\vartheta^{n}}(T)\right)\right] \leq \hat{g}_{J}(x) .
$$


To show this, it suffices to follow line by line the arguments contained after the equation (6.7) in the proof of Proposition 6.7 in [3].

Step 2. We now consider the case $I \cup J \neq \mathcal{K}$. We assume that

$$
y_{0}:=v_{I J}^{\lambda *}(T, x, p)>G^{\lambda}(x, p)
$$

and work towards a contradiction. It follows from Step 1 that $\hat{g}_{J^{\prime}}(x) \geq v_{J^{\prime} c}^{\lambda_{J^{\prime}}}(T, x)$. In view of (4.2) and (3.6), this leads to $v_{I J}^{\lambda *}(T, x, p)>v_{J^{\prime c} J^{\prime}}^{\lambda *}(T, x)$. Hence, there exists a sequence $\left(t_{n}, x_{n}, p_{n}\right)_{n} \subset D_{I J}$ which converges to $(T, x, p)$ such that $v_{I J}^{\lambda}\left(t_{n}, x_{n}, p_{n}\right) \rightarrow v_{I J}^{\lambda *}(T, x, p)$ and

$$
v_{J^{\prime} \mathcal{J} J^{\prime}}^{\lambda *}\left(t_{n}, x_{n}, p_{n}\right)<v_{J^{\prime c} J^{\prime}}^{\lambda *}(T, x, p)+\epsilon<y_{n} \text { for all } n \geq 1 \text {, for some } \epsilon>0,
$$

where $y_{n}:=v_{I J}^{\lambda}\left(t_{n}, x_{n}, p_{n}\right)-n^{-1}$. We can then find $\nu_{n} \in \mathcal{U}$ such that

$$
Y_{n}(T) \geq \hat{g}_{J^{\prime}}\left(X_{n}(T)\right) \geq \ell,
$$

where $\left(X_{n}, Y_{n}\right):=\left(X_{t_{n}, x_{n}}, Y_{t_{n}, x_{n}, y_{n}}^{\nu_{n}}\right)$. Moreover, since $\Delta_{\lambda}^{l}$ is strictly increasing on $\left\{\left(x^{\prime}, y^{\prime}\right): \Delta_{\lambda}^{l}\left(x^{\prime}, y^{\prime}\right) \in\right.$ $(0,1)\}$ and $y_{0}>G^{\lambda}(x, p)$, we have $\Delta_{\lambda}^{l}\left(x, y_{0}\right)>p^{l}$ for $l \notin I^{\prime} \cup J^{\prime}$. Since $\left(X_{n}(T), Y_{n}(T)\right) \rightarrow\left(x, y_{0}\right)$ in law, up to a subsequence, because $U$ is bounded and by the Lipschitz continuity of $\left(\mu_{X}, \sigma_{X}\right)$, we deduce that $\mathbb{E}\left[\Delta_{\lambda}^{l}\left(X_{n}(T), Y_{n}(T)\right)\right] \geq p_{n}^{l}$ for $l \notin I^{\prime} \cup J^{\prime}$, and $n$ large enough. Finally, $Y_{n}(T) \geq \ell$ so that $\mathbb{E}\left[\Delta_{\lambda}^{l}\left(X_{n}(T), Y_{n}(T)\right)\right] \geq 0$ for $l \in I^{\prime}$. This contradicts the fact that $y_{n}<v_{I J}^{\lambda}\left(t_{n}, x_{n}, p_{n}\right)$.

We now turn to the boundary condition in the $p$-variable, i.e. as $p \rightarrow \partial B_{I J}$.

Proposition 4.2 For all $(I, J),\left(I^{\prime}, J^{\prime}\right) \in \mathcal{P}_{\kappa}$ such that $\left(I^{\prime}, J^{\prime}\right) \supset(I, J)$, we have

$$
v_{I J}^{\lambda *} \leq v_{I^{\prime} J^{\prime}}^{\lambda^{*}} \text { on } D_{I^{\prime} J^{\prime}} .
$$

Proof. Since $v^{\lambda}$ is non-decreasing with respect to each variable $p^{i}, i \leq k$, we have $v_{I J}^{\lambda} \leq v_{I J^{\prime}}^{\lambda}$ for $J^{\prime} \supset J$. Hence it suffices to show the result for $J=J^{\prime}$. We also assume that $I^{\prime} \neq I$, since otherwise there is nothing to prove. Moreover, we claim that it is enough to show that

$$
v_{I J}^{\lambda *} \leq \bar{v}_{I J}^{I^{\prime}}:=\max \left\{v_{(I \cup K) J}^{\lambda *}: K \subset I^{\prime} \backslash I, K \neq \emptyset\right\} \text { on } D_{I^{\prime} J^{\prime}} .
$$

Indeed, if the above holds, then there exists $\hat{K}_{1} \subset I^{\prime} \backslash I$ such that $\hat{K}_{1} \neq \emptyset$ and $v_{I J}^{\lambda *} \leq v_{\left(I \cup \hat{K}_{1}\right) J}^{\lambda}$. If $\hat{K}_{1} \cup I=I^{\prime}$, the result is proved. If not, then applying the same result to $I \cup \hat{K}_{1}$ instead of $I$ implies that there exists $\tilde{K}_{1} \subset I^{\prime} \backslash\left(I \cup \hat{K}_{1}\right)$ such that $\hat{K}_{2}=\tilde{K}_{1} \cup \hat{K}_{1}$ strictly contains $\hat{K}_{1}$ and for which $v_{I J}^{\lambda *} \leq v_{\left(I \cup \hat{K}_{2}\right) J}^{\lambda *}$. The result then follows by iterating this procedures so as to construct an increasing sequence of sets $\hat{K}_{n} \subset I^{\prime} \backslash I$ such that $I \cup \hat{K}_{n}=I^{\prime}$ for a finite $n$.

We proceed in three steps.

Step 1. We first show that for any smooth function $\tilde{\varphi}$ on $\bar{D}$ and $(\tilde{t}, \tilde{x}, \tilde{p}) \in D_{I^{\prime} J}$ such that $D_{p^{i}} \tilde{\varphi}(\tilde{t}, \tilde{x}, \tilde{p}) \neq$ 0 for some $i \in(I \cup J)^{c}$ and

$$
\max _{\bar{D}_{I J}}(\text { strict })\left(v_{I J}^{\lambda *}-\tilde{\varphi}\right)=\left(v_{I J}^{\lambda *}-\tilde{\varphi}\right)(\tilde{t}, \tilde{x}, \tilde{p})=0
$$

we have

$$
\min \left\{\tilde{\varphi}-\bar{v}_{I J}^{I^{\prime}},-\partial_{t} \tilde{\varphi}+F_{I J *} \tilde{\varphi}\right\}(\tilde{t}, \tilde{x}, \tilde{p}) \leq 0 .
$$

Assume to the contrary that there exists $\eta>0$ s.t.

$$
\min \left\{\tilde{\varphi}-\bar{v}_{I J}^{I^{\prime}},-\partial_{t} \tilde{\varphi}+F_{I J *} \tilde{\varphi}\right\}(\tilde{t}, \tilde{x}, \tilde{p}) \geq 2 \eta
$$


In view of Remark 2.9, this implies that there exists $\varepsilon>0$ and a locally Lipschitz map $(\hat{u}, \hat{a})$ such that

$$
\begin{aligned}
& \min \left\{\tilde{\varphi}-\bar{v}_{I J}^{I^{\prime}},-\partial_{t} \tilde{\varphi}+L^{(\hat{u}, \hat{a})(\cdot, D \tilde{\varphi})}\left(\cdot, D \tilde{\varphi}, D^{2} \tilde{\varphi}\right)\right\}(t, x, p) \geq \eta, \\
& (\hat{u}, \hat{a})(x, D \tilde{\varphi}(t, x, p)) \in N_{I J}^{0}(x, D \tilde{\varphi}(t, x, p)),
\end{aligned}
$$

for all $(t, x, p) \in B:=B_{\varepsilon}(\tilde{t}, \tilde{x}, \tilde{p}) \cap \bar{D}_{I J}$.

Let $\left(t_{n}, x_{n}, p_{n}\right)$ be a sequence in $B$ that converges to $(\tilde{t}, \tilde{x}, \tilde{p})$ such that

$$
v_{I J}^{\lambda}\left(t_{n}, x_{n}, p_{n}\right) \rightarrow v_{I^{\prime} J^{\prime}}^{\lambda *}(\tilde{t}, \tilde{x}, \tilde{p})
$$

and set $y_{n}:=v_{I J}^{\lambda}\left(t_{n}, x_{n}, p_{n}\right)-n^{-1}$ so that

$$
\gamma_{n}:=y_{n}-\tilde{\varphi}\left(t_{n}, x_{n}, p_{n}\right) \rightarrow_{n \rightarrow \infty} 0 .
$$

We denote by $\left(X^{n}, P^{n}, Y^{n}\right)$ the solution of the (2.1)-(2.4) associated to the initial condition $\left(t_{n}, x_{n}, p_{n}\right)$ and the Markovian control

$$
\left(\nu^{n}, \alpha^{n}\right)=(\hat{u}, \hat{a})\left(X^{n}, D \tilde{\varphi}\left(\cdot, X^{n}, P^{n}\right)\right)
$$

and define the stopping time

$$
\theta_{n}:=\theta_{n 1} \wedge \theta_{n 2}
$$

where

$$
\theta_{n 1}:=\inf \left\{s \geq t_{n}: \min _{i \in I^{\prime} \backslash I} P^{n, i}(s)=0\right\}, \theta_{n 2}:=\inf \left\{s \geq t_{n}:\left(s, X^{n}(s), P^{n}(s)\right) \notin B \cap D_{I J}\right\} .
$$

Note that, since $(\tilde{t}, \tilde{x}, \tilde{p})$ achieves a strict local maximum of $v_{I J}^{\lambda *}-\tilde{\varphi}$, we have

$$
v_{I J}^{\lambda *}-\tilde{\varphi} \leq-\zeta \text { on } \partial B=\partial\left(B \cap D_{I J}\right), \text { for some } \zeta>0 .
$$

Using (4.6), we then deduce that

$$
\begin{aligned}
Y^{n}\left(\theta_{n}\right)-\gamma_{n} & \geq \tilde{\varphi}\left(\theta_{n}, X^{n}\left(\theta_{n}\right), P^{n}\left(\theta_{n}\right)\right) \\
& \geq\left(\bar{v}_{I J}^{I^{\prime}}\left(\theta_{n}, X^{n}\left(\theta_{n}\right), P^{n}\left(\theta_{n}\right)\right)+\eta\right) \mathbf{1}_{\theta_{n}=\theta_{n 1}}+\left(v_{I J}^{\lambda *}\left(\theta_{n}, X^{n}\left(\theta_{n}\right), P^{n}\left(\theta_{n}\right)\right)+\zeta\right) \mathbf{1}_{\theta_{n}<\theta_{n 1}} .
\end{aligned}
$$

We now observe that, by definition of $\theta_{n 1}$ and $\theta_{n 2},\left(\theta_{n 2}, X^{n}\left(\theta_{n 2}\right), P^{n}\left(\theta_{n 2}\right)\right) \in D_{I J}$ and therefore $v_{I J}^{\lambda}\left(\theta_{n}, X^{n}\left(\theta_{n}\right), P^{n}\left(\theta_{n}\right)\right)=v^{\lambda}\left(\theta_{n}, X^{n}\left(\theta_{n}\right), P^{n}\left(\theta_{n}\right)\right)$ on $\left\{\theta_{n}<\theta_{n 1}\right\}$. On the other hand, letting $K$ be the random subset of $I^{\prime} \backslash I$ such that $P^{n, i}\left(\theta_{n 1}\right)=0$ for $i \in K$, we have $\bar{v}_{I J}^{I^{\prime}}\left(\theta_{n}, X^{n}\left(\theta_{n}\right), P^{n}\left(\theta_{n}\right)\right) \geq$ $v_{(I \cup K) J}^{\lambda}\left(\theta_{n}, X^{n}\left(\theta_{n}\right), P^{n}\left(\theta_{n}\right)\right)=v^{\lambda}\left(\theta_{n}, X^{n}\left(\theta_{n}\right), P^{n}\left(\theta_{n}\right)\right)$ on $\left\{\theta_{n}=\theta_{n 1}\right\}$. It then follows from the previous inequality that

$$
Y^{n}\left(\theta_{n}\right)-\gamma_{n} \geq v^{\lambda}\left(\theta_{n}, X^{n}\left(\theta_{n}\right), P^{n}\left(\theta_{n}\right)\right)+\zeta \wedge \eta .
$$

Since $\gamma_{n} \rightarrow 0$, this leads to a contradiction to GDP2 for $n$ large.

Step 2. The rest of the proof is similar to the proof of Section 6.2 in [6]. We provide the main arguments for completeness. It remains to show that, for any smooth function $\bar{\varphi}$ and $(\tilde{t}, \tilde{x}, \tilde{p}) \in D_{I^{\prime} J}$ so that

$$
\max _{\bar{D}_{I^{\prime} J}}(\operatorname{strict})\left(v_{I J}^{\lambda *}-\bar{\varphi}\right)=v_{I J}^{\lambda *}(\tilde{t}, \tilde{x}, \tilde{p})-\bar{\varphi}(\tilde{t}, \tilde{x}, \tilde{p})=0,
$$

we have

$$
\bar{\varphi}(\tilde{t}, \tilde{x}, \tilde{p}) \leq \bar{v}_{I J}^{I^{\prime}}(\tilde{t}, \tilde{x}, \tilde{p})
$$


We argue by contradiction and assume that

$$
\bar{\varphi}(\tilde{t}, \tilde{x}, \tilde{p})>\bar{v}_{I J}^{I^{\prime}}(\tilde{t}, \tilde{x}, \tilde{p}) .
$$

Given $\rho>0$ and $k \geq 1$, we define the modified test function

$$
\varphi_{k}(t, x, p):=\bar{\varphi}(t, x, p)+|x-\tilde{x}|^{4}+|t-\tilde{t}|^{2}+\sum_{i \notin I \cup J}\left|p_{i}-\tilde{p}_{i}\right|^{4}+\sum_{i \in I^{\prime} \backslash I} \psi_{k}\left(1-p_{i}\right),
$$

where

$$
\psi_{k}(z):=-k \rho \int_{z}^{1} \frac{e^{2 k}}{e^{k(s+1)}-e^{2 k+1}} d s, \text { for all } z \in \mathbb{R},
$$

Let $\left(t_{k}, x_{k}, p_{k}\right) \in \bar{D}_{I J}$ be such that it maximizes of $\left(v_{I J}^{\lambda *}-\varphi_{k}\right)$ on $\bar{D}_{I J}$ and observe that

$$
\begin{gathered}
-2 \rho k \leq \psi_{k}^{\prime} \leq-\frac{\rho k}{2(e-1)}, \\
\psi^{\prime \prime}<0, \\
\lim _{k \rightarrow \infty} \frac{\left(\psi_{k}^{\prime}\left(z_{k}\right)\right)^{2}}{\left|\psi_{k} \prime\left(z_{k}\right)\right|}=\rho \text {, if }\left(z_{k}\right)_{k \geq 1} \subset(0,1) \text { is such that } \lim _{k \rightarrow \infty} k\left(1-z_{k}\right)=0 .
\end{gathered}
$$

Standard arguments then show that

$$
\left(t_{k}, x_{k}, p_{k}\right) \rightarrow(\tilde{t}, \tilde{x}, \tilde{p}) \text { and } k p_{k} \rightarrow 0,
$$

see e.g. Step 2 in Section 6.1 of [6]. Note that (4.11) implies that $D_{p^{i}} \varphi_{k}\left(t_{k}, x_{k}, p_{k}\right)<0$ for $i \in I^{\prime} \backslash I$, for $k$ large enough. It then follows from Step 1, (4.9) and (3.13) that

$$
-\partial_{t} \varphi_{k}\left(t_{k}, x_{k}, p_{k}\right)+F_{I J *} \varphi_{k}\left(t_{k}, x_{k}, p_{k}\right) \leq 0 .
$$

Then, there exist $\varepsilon_{k}, q_{k} \in \mathbb{R}^{d+\kappa}$ and $A_{k} \in \mathbb{M}^{d+\kappa}$ such that

$$
\begin{gathered}
\varepsilon_{k} \rightarrow 0 \\
\left|\left(q_{k}, A_{k}\right)-\left(D \varphi_{k}, D^{2} \varphi_{k}\right)\left(t_{k}, x_{k}, p_{k}\right)\right| \leq \frac{1}{k}, \\
-\partial_{t} \varphi_{k}\left(t_{k}, x_{k}, p_{k}\right)+F_{I J}^{\varepsilon_{k}}\left(x_{k}, p_{k}, q_{k}, A_{k}\right) \leq \frac{1}{k} .
\end{gathered}
$$

Given an arbitrary $u \in U$, fix $i_{0} \in I^{\prime} \backslash I$ and $\alpha_{k} \in \mathbb{M}^{\kappa, d}$ such that $\alpha_{k}^{j}=0$ for $j \neq i_{0}$ and

$$
\alpha_{k}^{i_{0} \cdot}:=\left(\sigma_{Y}\left(x_{k}, u\right)-q_{k}^{x}\left(t_{k}, x_{k}, p_{k}\right)^{\top} \sigma_{X}\left(x_{k}\right)\right) / q_{k}^{p^{i_{0}}},
$$

where $q_{k}^{x}$ stands for the first $d$ components of $q_{k}$ and $q_{k}^{p^{i_{0}}}$ stands for (by abuse of notations) the $d+i_{0}$ component of $q_{k}$. Note that $\left(u, \alpha_{k}\right) \in N_{I J}^{0}\left(x_{k}, q_{k}\right)$. Combined with the third inequality in (4.14), this implies that

$$
\begin{aligned}
k^{-1} \geq & -\partial_{t} \varphi_{k}\left(t_{k}, x_{k}, p_{k}\right)+\mu_{Y}\left(x_{k}, u\right)-\mu_{X}\left(x_{k}\right)^{\top} q_{k}^{x}-\frac{1}{2} \operatorname{Trace}\left[\sigma_{X}\left(x_{k}\right) \sigma_{X}\left(x_{k}\right)^{\top} A_{k}^{x x}\right] \\
& -\frac{1}{2}\left(\alpha_{k}^{i_{0} \cdot}\right)^{2} A_{k}^{p^{i_{0}} p^{i_{0}}}-\sigma_{X}\left(x_{k}\right)^{\top} A_{k}^{x p^{i_{0}}} \alpha_{k}^{i_{0} .}
\end{aligned}
$$

where $A_{k}^{x x}=\left(A_{k}^{i j}\right)_{i, j \leq d}, A_{k}^{p^{i_{0}} p^{i_{0}}}=A_{k}^{d+i_{0} d+i_{0}}$ and $A_{k}^{x p^{i_{0}}}=\left(A_{k}^{i d+i_{0}}\right)_{i \leq d}$. Sending $k \rightarrow \infty$, using (4.11), (4.13), the definition of $\alpha_{k}^{i_{0}} \cdot,(4.14)$ and recalling that $D_{p^{i_{0}}} \bar{\varphi}=0$ then leads to

$$
\begin{aligned}
0 \geq & -\partial_{t} \bar{\varphi}(\tilde{t}, \tilde{x}, \tilde{p})+\mu_{Y}(\tilde{x}, u)-\mu_{X}(\tilde{x})^{\top} D_{x} \bar{\varphi}(\tilde{t}, \tilde{x}, \tilde{p})-\frac{1}{2} \operatorname{Trace}\left[\sigma_{X}(\tilde{x}) \sigma_{X}(\tilde{x})^{\top} D_{x x} \varphi(\tilde{t}, \tilde{x}, \tilde{p})\right] \\
& +\frac{1}{2} \rho^{-1}\left|\sigma_{Y}(\tilde{x}, u)-D_{x} \varphi(\tilde{t}, \tilde{x}, \tilde{p})^{\top} \sigma_{X}(\tilde{x})\right|^{2} .
\end{aligned}
$$

Since $\rho>0$ and $u \in U$ are arbitrary, this implies that

$$
\left|u^{\top} \sigma(\tilde{x})-D_{x} \varphi(\tilde{t}, \tilde{x}, \tilde{p})^{\top} \sigma_{X}(\tilde{x})\right|^{2}=0 \text { for all } u \in U .
$$

This leads to a contradiction since $\sigma$ is assumed to be invertible. 


\subsubsection{Boundary condition for the lower-semicontinuous envelope}

We start with the boundary condition as $t \rightarrow T$.

Proposition 4.3 For all $(I, J),\left(I^{\prime}, J^{\prime}\right) \in \mathcal{P}_{\kappa}$ such that $\left(I^{\prime}, J^{\prime}\right) \supset(I, J)$, we have

$$
v_{I J *}^{\lambda}(T, \cdot) \geq G^{\lambda} \text { on }(0, \infty)^{d} \times \bar{B}_{I^{\prime} J^{\prime}} .
$$

Proof. Fix $(x, p) \in(0, \infty)^{d} \times \bar{B}_{I^{\prime} J^{\prime}}$. Since $v_{I J *}^{\lambda} \geq \ell$, the required result is trivial when $p=0$. We thus consider the case where $p \neq 0$, and fix $l \in \mathcal{K}$ such that that $p^{l}>0$. Let $\left(t_{n}, x_{n}, p_{n}\right)_{n} \subset D_{I J}$ be a sequence that converges to $(T, x, p)$ and such that $v_{I J}^{\lambda}\left(t_{n}, x_{n}, p_{n}\right) \rightarrow v_{I J *}^{\lambda}(T, x, p)$. We define $y_{n}:=v_{I J}^{\lambda}\left(t_{n}, x_{n}, p_{n}\right)+n^{-1}$ so that, for each $n$, there exists $\left(\nu^{n}, \alpha^{n}\right) \in \mathcal{U} \times \mathcal{A}_{t_{n}, p_{n}}$ satisfying $y_{n}+Y_{n}(T) \geq \ell$ and

$$
\mathbb{E}\left[\Delta_{\lambda}^{l}\left(X_{n}(T), Y_{n}(T)\right)\right] \geq p_{n}^{l},
$$

where $\left(X_{n}, Y_{n}\right):=\left(X_{t_{n}, x_{n}}, Y_{t_{n}, x_{n}, y_{n}}^{\nu_{n}}\right)$. Using the fact that $U$ is bounded and that $\left(\mu_{X}, \sigma_{X}\right)$ is Lipschitz continuous, one easily checks that, after possibly passing to a subsequence, $\left(X_{n}(T), Y_{n}(T)\right)$ converges to $\left(x, v_{I J *}^{\lambda}(T, x, p)\right) \mathbb{P}$-a.s. and in law. Since $\Delta^{\lambda}$ is continuous, this implies that

$$
\Delta_{\lambda}^{l}\left(x, v_{I J *}^{\lambda}(T, x, p)\right) \geq p^{l}>0 .
$$

By arbitrariness of $l$ such that $p^{l} \neq 0$, this leads to the required result.

In order to discuss the boundary condition in the $p$-variable, we follow [6] and first provide a supersolution property for $v_{I J}^{\lambda}$ on the boundary $\bar{D}_{I J} \cap D_{I^{\prime} J^{\prime}}$ for $\left(I^{\prime}, J^{\prime}\right) \supset(I, J)$. A more precise statement will be deduced from the following one and the comparison result of Proposition 4.6 below, see Section 4.4.

Proposition 4.4 For all $(I, J),\left(I, J^{\prime}\right) \in \mathcal{P}_{\kappa}$ such that $J^{\prime} \supset J, v_{I J *}^{\lambda}$ is a supersolution on $\bar{D}_{I J}$ of

$$
\min \left\{\varphi-\ell,-\partial_{t} \varphi+F_{I J^{\prime}}^{*} \varphi\right\} \geq 0 \quad \text { on } \quad D_{I J^{\prime}} .
$$

Proof. By definition, we have $v_{I J *}^{\lambda} \geq \ell$. The rest of proof is divided in several steps.

Step 1. We first show that, for a smooth function $\tilde{\varphi}$ on $\bar{D}_{I J}$ and $(\tilde{t}, \tilde{x}, \tilde{p}) \in \bar{D}_{I J} \cap D_{I J^{\prime}}$ so that

$$
\min (\text { strict })_{\bar{D}_{I J}}\left(v_{*}^{\lambda}-\tilde{\varphi}\right)=\left(v_{*}^{\lambda}-\tilde{\varphi}\right)(\tilde{t}, \tilde{x}, \tilde{p})=0,
$$

we have

$$
\max \left\{\tilde{\varphi}-\underline{v}_{I J}^{J^{\prime}},-\partial_{t} \tilde{\varphi}+F_{I J}^{*} \tilde{\varphi}\right\}(\tilde{t}, \tilde{x}, \tilde{p}) \geq 0
$$

where

$$
v_{I J *}^{\lambda} \geq \underline{v}_{I J}^{J^{\prime}}:=\min \left\{v_{I(J \cup K) *}^{\lambda}: K \subset J^{\prime} \backslash J, K \neq \emptyset\right\} .
$$

We argue by contradiction and assume that there exists $\varepsilon, \eta>0$ such that

$$
\begin{gathered}
\max \left\{\tilde{\varphi}-\underline{v}_{I J}^{J^{\prime}},-\partial_{t} \tilde{\varphi}+F_{I J}^{*} \tilde{\varphi}\right\}(t, x, p) \leq-\eta, \\
\forall(t, x, p) \in B:=B_{\varepsilon}(\tilde{t}, \tilde{x}, \tilde{p}) \cap \bar{D}_{I J} .
\end{gathered}
$$

Note that, since $(\tilde{t}, \tilde{x}, \tilde{p})$ achieves a strict local minimum of $v_{I J *}^{\lambda}-\tilde{\varphi}$ on $\bar{D}_{I J}$, we have

$$
v_{I J *}^{\lambda}-\tilde{\varphi} \geq \zeta \text { on } \partial B=\partial\left(B \cap D_{I J}\right),
$$

for some $\zeta>0$. Let $\left(t_{n}, x_{n}, p_{n}\right)$ be a sequence in $B \cap D_{I J}$ that converges to $(\tilde{t}, \tilde{x}, \tilde{p})$ such that

$$
v_{I J}^{\lambda}\left(t_{n}, x_{n}, p_{n}\right) \rightarrow v_{I J *}^{\lambda}(\tilde{t}, \tilde{x}, \tilde{p})
$$


and set $y_{n}:=v_{I J}^{\lambda}\left(t_{n}, x_{n}, p_{n}\right)+n^{-1}$ so that

$$
\gamma_{n}:=y_{n}-\tilde{\varphi}\left(t_{n}, x_{n}, p_{n}\right) \rightarrow 0 .
$$

Since $y^{n}>v_{I J}\left(t_{n}, x_{n}, p_{n}\right)$, there exists $\left(\nu^{n}, \alpha^{n}\right) \in \mathcal{U} \times \mathcal{A}_{t_{n}, p_{n}}$ such that $\Delta_{\lambda}\left(X^{n}(T), Y^{n}(T)\right) \geq P^{n}(T)$, where $\left(Y^{n}, X^{n}, P^{n}\right):=\left(Y_{t_{n}, x_{n}, y_{n}}^{\nu^{n}}, X_{t_{n}, x_{n}}, P_{t_{n}, x_{n}}^{\alpha^{n}}\right)$.

Let us now define

$$
\theta_{n}:=\theta_{n 1} \wedge \theta_{n 2}
$$

where

$$
\theta_{n 1}:=\inf \left\{s \geq t_{n}: \max _{i \in J^{\prime} \backslash J} P^{n, i}(s)=1\right\}, \theta_{n 2}:=\inf \left\{s \geq t_{n}:\left(s, X^{n}(s), P^{n}(s)\right) \notin B \cap D_{I J}\right\} .
$$

It then follows from GDP1 that

$$
Y^{n}\left(\theta_{n}\right) \geq v^{\lambda}\left(\theta_{n}, X^{n}\left(\theta_{n}\right), P^{n}\left(\theta_{n}\right)\right) .
$$

We now observe that, by definition of $\theta_{n 1}$ and $\theta_{n 2},\left(\theta_{n}, X^{n 2}\left(\theta_{n 2}\right), P^{n}\left(\theta_{n 2}\right)\right) \in D_{I J}$ and therefore

$$
v_{I J}^{\lambda}\left(\theta_{n}, X^{n}\left(\theta_{n}\right), P^{n}\left(\theta_{n}\right)\right)=v^{\lambda}\left(\theta_{n}, X^{n}\left(\theta_{n}\right), P^{n}\left(\theta_{n}\right)\right) \text { on }\left\{\theta_{n}<\theta_{n 1}\right\} .
$$

On the other hand, letting $K$ be the random subset of $J^{\prime} \backslash J$ such that $P^{n, i}\left(\theta_{n 1}\right)=1$ for $i \in K$, we have $\underline{v}_{I J}^{J^{\prime}}\left(\theta_{n}, X^{n}\left(\theta_{n}\right), P^{n}\left(\theta_{n}\right)\right) \leq v_{I(J \cup K)}^{\lambda}\left(\theta_{n}, X^{n}\left(\theta_{n}\right), P^{n}\left(\theta_{n}\right)\right)=v^{\lambda}\left(\theta_{n}, X^{n}\left(\theta_{n}\right), P^{n}\left(\theta_{n}\right)\right)$ on $\left\{\theta_{n}=\theta_{n 1}\right\}$. It then follows from the previous inequality that

$$
Y^{n}\left(\theta_{n}\right) \geq v_{I J}^{\lambda}\left(\theta_{n}, X^{n}\left(\theta_{n}\right), P^{n}\left(\theta_{n}\right)\right) \mathbf{1}_{\theta_{n}<\theta_{n 1}}+\underline{v}_{I J}^{J^{\prime}}\left(\theta_{n}, X^{n}\left(\theta_{n}\right), P^{n}\left(\theta_{n}\right)\right) \mathbf{1}_{\theta_{n}=\theta_{n 1}} .
$$

We now appeal to (4.18) and (4.19) to deduce that

$$
Y^{n}\left(\theta_{n}\right) \geq \tilde{\varphi}\left(\theta_{n}, X^{n}\left(\theta_{n}\right), P^{n}\left(\theta_{n}\right)\right)+\zeta \wedge \eta .
$$

The required contradiction then follows from the same arguments as in Section 5.1 of [6].

Step 2. We now show that for any smooth function $\tilde{\varphi}$ on $\bar{D}_{I J}$ and $(\tilde{t}, \tilde{x}, \tilde{p}) \in \bar{D}_{I J} \cap D_{I J^{\prime}}$ such that

$$
\min (\text { strict })_{\bar{D}_{I J}}\left(v_{*}^{\lambda}-\tilde{\varphi}\right)=\left(v_{*}^{\lambda}-\tilde{\varphi}\right)(\tilde{t}, \tilde{x}, \tilde{p})=0,
$$

we have

$$
\max \left\{\tilde{\varphi}-v_{I J^{\prime} *}^{\lambda},-\partial_{t} \tilde{\varphi}+F_{I J}^{*} \tilde{\varphi}\right\}(\tilde{t}, \tilde{x}, \tilde{p}) \geq 0
$$

To see this, assume that

$$
\left(-\partial_{t} \tilde{\varphi}+F_{I J}^{*} \tilde{\varphi}\right)(\tilde{t}, \tilde{x}, \tilde{p})<0 .
$$

Then, it follows from Step 1 that $v_{I J *}^{\lambda}(\tilde{t}, \tilde{x}, \tilde{p})=\tilde{\varphi}(\tilde{t}, \tilde{x}, \tilde{p}) \geq v_{I\left(J \cup K_{1}\right) *}^{\lambda}(\tilde{t}, \tilde{x}, \tilde{p})$ for some $K_{1} \subset J^{\prime} \backslash J$ such that $K_{1} \neq \emptyset$. If $J \cup K_{1}=J^{\prime}$, then this proves the required result. If not, then we use the fact that $v$ is non-decreasing in its $p^{i}$ components to deduce that $v_{I J *}^{\lambda} \leq v_{I\left(J \cup K_{1}\right) *}^{\lambda}$. It follows that $v_{I J *}^{\lambda}(\tilde{t}, \tilde{x}, \tilde{p})=$ $v_{I\left(J \cup K_{1}\right) *}^{\lambda}(\tilde{t}, \tilde{x}, \tilde{p})$ and that $(\tilde{t}, \tilde{x}, \tilde{p})$ is also a minimum point of $v_{I\left(J \cup K_{1}\right) *}^{\lambda}-\tilde{\varphi}$ on $\bar{D}_{I\left(J \cup K_{1}\right)} \subset \bar{D}_{I J^{\prime}}$. In view of Step 1, this implies that

$$
\max \left\{\tilde{\varphi}-\underline{v}_{I\left(J \cup K_{1}\right)}^{J^{\prime}},-\partial_{t} \tilde{\varphi}+F_{I\left(J \cup K_{1}\right)}^{*} \tilde{\varphi}\right\}(\tilde{t}, \tilde{x}, \tilde{p}) \geq 0,
$$

which, by (4.21) and the inequality $F_{I\left(J \cup K_{1}\right)}^{*} \leq F_{I J}^{*}$, implies that $\tilde{\varphi}(\tilde{t}, \tilde{x}, \tilde{p}) \geq \underline{v}_{I\left(J \cup K_{1}\right)}^{\lambda J^{\prime}}(\tilde{t}, \tilde{x}, \tilde{p})$. After at most $\kappa$ iterations of this argument, we finally obtain $\tilde{\varphi}(\tilde{t}, \tilde{x}, \tilde{p}) \geq v_{I J^{\prime} *}(\tilde{t}, \tilde{x}, \tilde{p})$. 
Step 3. Repeating the arguments of Section 6.1 of [6], we then deduce from Step 2 that, for any smooth function $\tilde{\varphi}$ on $\bar{D}_{I J^{\prime}}$ and $(\tilde{t}, \tilde{x}, \tilde{p}) \in D_{I J^{\prime}} \cap \bar{D}_{I J}$ such that

$$
\min (\text { strict })_{\bar{D}_{I J}}\left(v_{I J *}^{\lambda}-\tilde{\varphi}\right)=\left(v_{I J *}^{\lambda}-\tilde{\varphi}\right)(\tilde{t}, \tilde{x}, \tilde{p})=0
$$

we have

$$
\max \left\{\tilde{\varphi}-v_{I J^{\prime} *}^{\lambda},-\partial_{t} \tilde{\varphi}+F_{I J^{\prime}}^{*} \tilde{\varphi}\right\}(\tilde{t}, \tilde{x}, \tilde{p}) \geq 0 .
$$

If $v_{I J *}^{\lambda}(\tilde{t}, \tilde{x}, \tilde{p})=\tilde{\varphi}(\tilde{t}, \tilde{x}, \tilde{p})<v_{I J^{\prime} *}^{\lambda}(\tilde{t}, \tilde{x}, \tilde{p})$ then

$$
\left(-\partial_{t} \tilde{\varphi}+F_{I J^{\prime}}^{*} \tilde{\varphi}\right)(\tilde{t}, \tilde{x}, \tilde{p}) \geq 0 .
$$

Otherwise, $v_{I J *}^{\lambda}(\tilde{t}, \tilde{x}, \tilde{p})=\tilde{\varphi}(\tilde{t}, \tilde{x}, \tilde{p})=v_{I J^{\prime} *}^{\lambda}(\tilde{t}, \tilde{x}, \tilde{p})$ so that $(\tilde{t}, \tilde{x}, \tilde{p})$ is a local minimizer of $v_{I J^{\prime} *}^{\lambda}-\tilde{\varphi}$ on $\bar{D}_{I J} \supset \bar{D}_{I J^{\prime}}$. In this case, we then deduce from (3.13) that (4.24) holds too.

\subsection{Gradient estimates}

In this section, we prove Proposition 3.4. It is based on the following growth estimate.

Proposition 4.5 Fix $(I, J),\left(I^{\prime}, J^{\prime}\right) \in \mathcal{P}_{\kappa}$ such that $I \cup J \neq \mathcal{K}$ and $J \subset J^{\prime}$. Let $(t, x, p) \in D_{I J}$ be such that $\left(v_{I J}^{\lambda}-v_{I^{\prime} J^{\prime}}^{\lambda}\right)(t, x, p)>\iota \geq 0$. Let $\varrho>0$ be defined as in (3.8). Then,

$$
v_{I J}^{\lambda}(t, x, p)-v_{I J}^{\lambda}\left(t, x, p \ominus C_{\lambda} \delta(\iota+\varrho) \mathbf{1}_{I J}\right) \geq \delta \iota \text { for all } 0 \leq \delta \leq 1,
$$

where $\mathbf{1}_{I J}$ stands for $\left(\mathbf{1}_{\{i \notin I \cup J\}}\right)_{i \leq \kappa}$.

Proof. Fix $(t, x, p) \in D_{I J}, y>v_{I J}^{\lambda}(t, x, p)$ and $\iota \geq 0$ such that $v_{I J}^{\lambda}(t, x, p)-\iota>v_{I^{\prime} J^{\prime}}^{\lambda}(t, x, p)$. Then, we can find $\nu \in \mathcal{U}$ such that $Y_{t, x, y}^{\nu}(T) \geq \ell$ and $\mathbb{E}\left[\Delta_{\lambda}^{i}\left(X_{t, x}(T), Y_{t, x, y}^{\nu}(T)\right)\right] \geq p^{i}$ for all $i \leq \kappa$, and $\nu^{\prime} \in \mathcal{U}$ such that $Y_{t, x, y-\iota}^{\nu^{\prime}}(T) \geq \ell$ and $Y_{t, x, y-\iota}^{\nu^{\prime}}(T) \geq \hat{g}^{j}\left(X_{t, x}(T)\right)$ for all $j \in J^{\prime}$, recall (3.1). Set $\nu_{\delta}:=(1-\delta) \nu+\delta \nu^{\prime} \in \mathcal{U}$, recall that $U$ is convex, and $y_{\delta}:=(1-\delta) y+\delta(y-\iota)=y-\delta \iota$.

Note that

$$
Y_{t, x, y \delta}^{\nu_{\delta}}(T)=(1-\delta) Y_{t, x, y}^{\nu}(T)+\delta Y_{t, x, y-\iota}^{\nu^{\prime}}(T),
$$

by (2.4). Combined with the above inequalities and the fact that $p^{i}=1$ for $i \in J \subset J^{\prime}$, this readily implies that

$$
Y_{t, x, y_{\delta}}^{\nu_{\delta}}(T) \geq \ell \text { and } Y_{t, x, y_{\delta}}^{\nu_{\delta}}(T) \geq \hat{g}^{i}\left(X_{t, x}(T)\right) \text { for } i \in J
$$

Since $\Delta_{\lambda}^{i}$ is $C_{\lambda}$-Lipschitz with respect to $y$, see (3.4), we also have

$$
\begin{aligned}
\mathbb{E}\left[\Delta_{\lambda}^{i}\left(X_{t, x}(T), Y_{t, x, y \delta}^{\nu_{\delta}}(T)\right)\right] & =\mathbb{E}\left[\Delta_{\lambda}^{i}\left(X_{t, x}(T), Y_{t, x, y}^{\nu}(T)+\delta\left(Y_{t, x, y-\iota}^{\nu^{\prime}}(T)-Y_{t, x, y}^{\nu}(T)\right)\right)\right] \\
& \geq \mathbb{E}\left[\Delta_{\lambda}^{i}\left(X_{t, x}(T), Y_{t, x, y}^{\nu}(T)\right)\right]-C_{\lambda} \delta \mathbb{E}\left[\left|Y_{t, x, y-\iota}^{\nu^{\prime}}(T)-Y_{t, x, y}^{\nu}(T)\right|\right] \\
& \geq p^{i}-C_{\lambda} \delta \mathbb{E}\left[\left|Y_{t, x, y-\iota}^{\nu^{\prime}}(T)-Y_{t, x, y}^{\nu}(T)\right|\right], \text { for } i \notin J,
\end{aligned}
$$

where

$$
\mathbb{E}\left[\left|Y_{t, x, y-\iota}^{\nu^{\prime}}(T)-Y_{t, x, y}^{\nu}(T)\right|\right] \leq \iota+\mathbb{E}\left[\left|\int_{t}^{T}\left(\nu_{s}-\nu_{s}^{\prime}\right)^{\top} \mu\left(X_{t, x}(s)\right) d s+\int_{t}^{T}\left(\nu_{s}-\nu_{s}^{\prime}\right)^{\top} \sigma\left(X_{t, x}(s)\right) d W_{s}\right|\right] .
$$

Recalling (2.2) and (2.3), standard estimates imply that the right-hand side term is bounded by $\varrho$ as defined in (3.8). Hence

$$
\mathbb{E}\left[\Delta_{\lambda}^{i}\left(X_{t, x}(T), Y_{t, x, y_{\delta}}^{\nu_{\delta}}(T)\right)\right] \geq p^{i}-C_{\lambda} \delta(\iota+\varrho), \text { for } i \notin J
$$


We now combine (4.26) and (4.27) to deduce that

$$
y_{\delta} \geq v_{I J}^{\lambda}\left(t, x, p \ominus C_{\lambda} \delta(\iota+\varrho) \mathbf{1}_{I J}\right) .
$$

By arbitrariness of $y>v_{I J}^{\lambda}(t, x, p)$, this implies the required result.

Proof of Proposition 3.4. Fix $(I, J) \in \mathcal{P}_{\kappa}$ and $(t, x, p) \in D_{I J}$ such that $v_{I J}^{\lambda *}(t, x, p)>v_{J^{c} J}^{\lambda *}(t, x, p)+\iota$ for some $\iota \geq 0$. Let $\varphi$ be a smooth function and assume that $(t, x, p)$ achieves the maximum of $v_{I J}^{\lambda *}-\varphi$. Since $v_{I J}^{\lambda}$ is non-decreasing with respect to its $p$-variable, and by definition of $v_{J^{c} J}^{\lambda^{*}}$, there exists $\left(t_{n}, x_{n}, p_{n}\right) \rightarrow(t, x, p)$ such that $v_{I J}^{\lambda}\left(t_{n}, x_{n}, p_{n}\right) \rightarrow v_{I J}^{\lambda *}(t, x, p)$ and $v_{I J}^{\lambda}\left(t_{n}, x_{n}, p_{n}+C_{\lambda} \delta(\iota+\varrho) \mathbf{1}_{I J}\right)>$ $v_{J^{c} J}^{\lambda}\left(t_{n}, x_{n}, p_{n}+C_{\lambda} \delta(\iota+\varrho) \mathbf{1}_{I J}\right)+\iota$ for $\delta>0$ small enough. By applying Proposition 4.5 at the point $\left(t_{n}, x_{n}, p_{n}+C_{\lambda} \delta(\iota+\varrho) \mathbf{1}_{I J}\right)$ for $\left(I^{\prime}, J^{\prime}\right)=\left(J^{c}, J\right)$, we deduce that

$$
v_{I J}^{\lambda}\left(t_{n}, x_{n}, p_{n}+C_{\lambda} \delta(\iota+\varrho) \mathbf{1}_{I J}\right)-v_{I J}^{\lambda}\left(t_{n}, x_{n}, p_{n}\right) \geq \delta \iota,
$$

and therefore

$$
\varphi\left(t, x, p+C_{\lambda} \delta(\iota+\varrho) \mathbf{1}_{I J}\right)-\varphi(t, x, p) \geq \delta \iota .
$$

Dividing by $\delta$ and sending $\delta$ to 0 leads to the required result for $\varpi$ defined as in (3.9) above.

\subsection{Comparison results}

We first provide a comparison result for $\left(\mathrm{S}^{\lambda}\right)$. Additional technical improvements will be considered in the next section to discuss the convergence of the numerical scheme defined in Section 3.3.

\subsubsection{For the system of PDEs $\left(\mathrm{S}^{\lambda}\right)$}

Proposition 4.6 Let $\psi_{1} \geq \psi_{2}$ be two functions such that $\psi_{1}$ and $-\psi_{2}$ are lower-semicontinuous. Fix $(I, J) \in \mathcal{P}_{\kappa}$. Let $V_{1}$ be a bounded lower-semicontinuous viscosity supersolution of

$$
H_{I J}^{\lambda *}\left[\varphi, \psi_{1}\right]=0 \text { on } D_{I J}
$$

and let $V_{2}$ be a bounded upper-semicontinuous viscosity subsolution of

$$
H_{I J *}^{\lambda}\left[\varphi, \psi_{2}\right]=0 \text { on } D_{I J}
$$

Assume that $V_{1} \geq V_{2}$ on $\partial D_{I J}$. Assume further that either $V_{1} \geq \psi_{2}$ on $D_{I J}$ or that $(I, J) \in \mathcal{P}_{\kappa}^{\kappa}$. Then, $V_{1} \geq V_{2}$ on $\bar{D}_{I J}$.

Proof.

Part 1: $(I, J) \notin \mathcal{P}_{\kappa}^{\kappa}$. As usual, we first fix $\rho>0$ and introduce the functions $\tilde{V}_{1}(t, x, p):=e^{\rho t} V_{1}(t, x, p)$ and $\tilde{V}_{2}(t, x, p):=e^{\rho t} V_{2}(t, x, p)$. Arguing by contradiction, we assume that

$$
\sup _{\bar{D}_{I J}}\left(\tilde{V}_{2}-\tilde{V}_{1}\right)=: m>0 .
$$

and work towards a contradiction.

1. For $n, k \geq 1$ and $\varepsilon>0$, we then define the function $\Psi_{n, \varepsilon}^{k}$ on $[0, T] \times \mathbb{R}^{2 n} \times[0,1]^{2 \kappa}$ by

$$
\Psi_{n, \varepsilon}^{k}(t, x, y, p, q):=\tilde{V}_{2}(t, x, p)-\tilde{V}_{1}(t, y, q)-\Theta_{n, \varepsilon}^{k}(t, x, y, p, q)
$$


where

$$
\Theta_{n, \varepsilon}^{k}(t, x, y, p, q):=\frac{n^{2}}{2}|x-y|^{2}+\frac{k^{2}}{2}|p-q|^{2}+f_{\varepsilon}(x),
$$

with

$$
f_{\varepsilon}(x):=\varepsilon\left(|x|^{2}+\sum_{i \leq d}\left(x^{i}\right)^{-1}\right) .
$$

It follows from the boundedness of $\tilde{V}_{2}$ and $\tilde{V}_{1}$ that $\Psi_{n, \varepsilon}^{k}$ achieves its maximum at some $\left(t_{n, \varepsilon}^{k}, x_{n, \varepsilon}^{k}, y_{n, \varepsilon}^{k}, p_{n, \varepsilon}^{k}, q_{n, \varepsilon}^{k}\right) \in$ $[0, T] \times(0, \infty)^{2 d} \times \bar{B}_{I J}^{2}$. Similarly, the map

$$
(t, x, p, q) \in[0, T] \times(0, \infty)^{d} \times \bar{B}_{I J}^{2} \mapsto \tilde{V}_{2}(t, x, p)-\tilde{V}_{1}(t, x, q)-\frac{k^{2}}{2}|p-q|^{2}-f_{\varepsilon}(x)
$$

achieves a maximum at some $\left(t_{\varepsilon}^{k}, x_{\varepsilon}^{k}, p_{\varepsilon}^{k}, q_{\varepsilon}^{k}\right) \in[0, T] \times(0, \infty)^{d} \times \bar{B}_{I J}^{2}$. Moreover, the inequality

$$
\Psi_{n, \varepsilon}^{k}\left(t_{n, \varepsilon}^{k}, x_{n, \varepsilon}^{k}, y_{n, \varepsilon}^{k}, p_{n, \varepsilon}^{k}, q_{n, \varepsilon}^{k}\right) \geq \Psi_{n, \varepsilon}^{k}\left(t_{\varepsilon}^{k}, x_{\varepsilon}^{k}, x_{\varepsilon}^{k}, p_{\varepsilon}^{k}, q_{\varepsilon}^{k}\right)
$$

implies that

$$
\begin{aligned}
\tilde{V}_{2}\left(t_{n, \varepsilon}^{k}, x_{n, \varepsilon}^{k}, p_{n, \varepsilon}^{k}\right)-\tilde{V}_{1}\left(t_{n, \varepsilon}^{k}, y_{n, \varepsilon}^{k}, q_{n, \varepsilon}^{k}\right) \geq & \tilde{V}_{2}\left(t_{\varepsilon}^{k}, x_{\varepsilon}^{k}, p_{\varepsilon}^{k}\right)-\tilde{V}_{1}\left(t_{\varepsilon}^{k}, x_{\varepsilon}^{k}, q_{\varepsilon}^{k}\right)-\frac{k^{2}}{2}\left|p_{\varepsilon}^{k}-q_{\varepsilon}^{k}\right|^{2}-f_{\varepsilon}\left(x_{\varepsilon}^{k}\right) \\
& +\frac{n^{2}}{2}\left|x_{n, \varepsilon}^{k}-y_{n, \varepsilon}^{k}\right|^{2}+\frac{k^{2}}{2}\left|p_{n, \varepsilon}^{k}-q_{n, \varepsilon}^{k}\right|^{2}+f_{\varepsilon}\left(x_{n, \varepsilon}^{k}\right) .
\end{aligned}
$$

Using the boundedness of $\tilde{V}_{2}$ and $\tilde{V}_{1}$ again together with the fact that $\bar{B}_{I J}$ is compact, we deduce that the term on the second line is bounded in $n$ so that, up to a subsequence,

$$
\left(t_{n, \varepsilon}^{k}, x_{n, \varepsilon}^{k}, y_{n, \varepsilon}^{k}, p_{n, \varepsilon}^{k}, q_{n, \varepsilon}^{k}\right) \rightarrow\left(\bar{t}_{\varepsilon}^{k}, \bar{x}_{\varepsilon}^{k}, \bar{x}_{\varepsilon}^{k}, \bar{p}_{\varepsilon}^{k}, \bar{q}_{\varepsilon}^{k}\right) \text { as } n \rightarrow \infty,
$$

for some $\left(\bar{t}_{\varepsilon}^{k}, \bar{x}_{\varepsilon}^{k}, \bar{p}_{\varepsilon}^{k}, \bar{q}_{\varepsilon}^{k}\right) \in[0, T] \times(0, \infty)^{d} \times \bar{B}_{I J}^{2}$. By sending $n \rightarrow \infty$ in the previous inequality, we also obtain

$$
\begin{aligned}
& \tilde{V}_{2}\left(t_{\varepsilon}^{k}, x_{\varepsilon}^{k}, p_{\varepsilon}^{k}\right)-\tilde{V}_{1}\left(t_{\varepsilon}^{k}, x_{\varepsilon}^{k}, q_{\varepsilon}^{k}\right)-\frac{k^{2}}{2}\left|p_{\varepsilon}^{k}-q_{\varepsilon}^{k}\right|^{2}-f_{\varepsilon}\left(x_{\varepsilon}^{k}\right) \\
& \leq \tilde{V}_{2}\left(\bar{t}_{\varepsilon}^{k}, \bar{x}_{\varepsilon}^{k}, \bar{p}_{\varepsilon}^{k}\right)-\tilde{V}_{1}\left(\bar{t}_{\varepsilon}^{k}, \bar{x}_{\varepsilon}^{k}, \bar{q}_{\varepsilon}^{k}\right)-\frac{k^{2}}{2}\left|\bar{p}_{\varepsilon}^{k}-\bar{q}_{\varepsilon}^{k}\right|^{2}-f_{\varepsilon}\left(\bar{x}_{\varepsilon}^{k}\right)-\liminf _{n \rightarrow \infty} \frac{n^{2}}{2}\left|x_{n, \varepsilon}^{k}-y_{n, \varepsilon}^{k}\right|^{2} .
\end{aligned}
$$

It then follows from the maximum property at $\left(t_{\varepsilon}^{k}, x_{\varepsilon}^{k}, p_{\varepsilon}^{k}, q_{\varepsilon}^{k}\right)$ that the last term on the right-hand side converges to 0 and that we can assume, without loss of generality, that $\left(\bar{t}_{\varepsilon}^{k}, \bar{x}_{\varepsilon}^{k}, \bar{p}_{\varepsilon}^{k}, \bar{q}_{\varepsilon}^{k}\right)=\left(t_{\varepsilon}^{k}, x_{\varepsilon}^{k}, p_{\varepsilon}^{k}, q_{\varepsilon}^{k}\right)$, i.e.

$$
\left(t_{n, \varepsilon}^{k}, x_{n, \varepsilon}^{k}, y_{n, \varepsilon}^{k}, p_{n, \varepsilon}^{k}, q_{n, \varepsilon}^{k}\right) \underset{n \rightarrow \infty}{\longrightarrow}\left(t_{\varepsilon}^{k}, x_{\varepsilon}^{k}, x_{\varepsilon}^{k}, p_{\varepsilon}^{k}, q_{\varepsilon}^{k}\right) \text { and } n^{2}\left|x_{n, \varepsilon}^{k}-y_{n, \varepsilon}^{k}\right|^{2} \underset{n \rightarrow \infty}{\longrightarrow} 0 .
$$

It follows from similar arguments that we could choose $\left(x_{\varepsilon}^{k}\right)_{\varepsilon>0}$ such that, up to a subsequence,

$$
f_{\varepsilon}\left(x_{\varepsilon}^{k}\right) \underset{\varepsilon \rightarrow 0}{\longrightarrow} 0 \text { and }\left(t_{\varepsilon}^{k}, p_{\varepsilon}^{k}, q_{\varepsilon}^{k}\right) \underset{\varepsilon \rightarrow 0}{\longrightarrow}\left(t_{k}, p_{k}, q_{k}\right),
$$

and

$$
\lim _{\varepsilon \rightarrow 0} \lim _{n \rightarrow \infty} \Psi_{n, \varepsilon}^{k}\left(t_{n, \varepsilon}^{k}, x_{n, \varepsilon}^{k}, y_{n, \varepsilon}^{k}, p_{n, \varepsilon}^{k}, q_{n, \varepsilon}^{k}\right)=\sup _{[0, T] \times(0, \infty)^{d} \times[0,1]^{2 \kappa}} \Psi_{0,0}^{k}(t, x, x, p, q) \geq m .
$$

For later use, note that the left-hand side in (4.31) together with the definition of $\left(\mu_{X}, \sigma_{X}\right)$ and the fact that $(\mu, \sigma)$ is bounded implies

$$
\left|D_{x} f_{\varepsilon}\left(x_{\varepsilon}^{k}\right)^{\top} \mu_{X}\left(x_{\varepsilon}^{k}\right)\right|+\left|D_{x} f_{\varepsilon}\left(x_{\varepsilon}^{k}\right)^{\top} \sigma_{X}\left(x_{\varepsilon}^{k}\right)\right|+\left|\operatorname{Trace}\left[\sigma_{X} \sigma_{X}^{\top}\left(x_{\varepsilon}^{k}\right) D_{x}^{2} f_{\varepsilon}\left(x_{\varepsilon}^{k}\right)\right]\right| \underset{\varepsilon \rightarrow 0}{\longrightarrow} 0 .
$$


Similarly, we must have

$$
\lim _{k \rightarrow \infty} k^{2}\left|p_{k}-q_{k}\right|^{2}=0
$$

Since $V_{1}(T, \cdot) \geq V_{2}(T, \cdot)$, the above implies that we can not have $t_{n, \varepsilon}^{k}=T$ along a subsequence. Since $V_{2} \geq V_{1}$ on $\partial D_{I J}$, we obtain a similar contradiction if, up to a subsequence, $\left(t_{n, \varepsilon}^{k}, x_{n, \varepsilon}^{k}, p_{n, \varepsilon}^{k}\right)_{\varepsilon, k, n} \in \partial D_{I J}$ or $\left(t_{n, \varepsilon}^{k}, y_{n, \varepsilon}^{k}, q_{n, \varepsilon}^{k}\right)_{\varepsilon, k, n} \in \partial D_{I J}$ for all $\varepsilon, n, k$. We can therefore assume from now on that $t_{n, \varepsilon}^{k}<T$, $\left(t_{n, \varepsilon}^{k}, x_{n, \varepsilon}^{k}, p_{n, \varepsilon}^{k}\right)_{\varepsilon, k, n} \notin \partial D_{I J}$ and $\left(t_{n, \varepsilon}^{k}, y_{n, \varepsilon}^{k}, q_{n, \varepsilon}^{k}\right)_{\varepsilon, k, n} \notin \partial D_{I J}$ for all $k, n, \varepsilon$.

2. For ease of notations, we now set $z_{n, \varepsilon}^{k}:=\left(t_{n, \varepsilon}^{k}, x_{n, \varepsilon}^{k}, y_{n, \varepsilon}^{k}, p_{n, \varepsilon}^{k}, q_{n, \varepsilon}^{k}\right)$. From Ishii's Lemma, see Theorem 8.3 in [8], we deduce that, for each $\eta>0$, there are real coefficients $a_{n, \varepsilon}^{k}, b_{n, \varepsilon}^{k}$ and symmetric matrices $\mathcal{X}_{n, \varepsilon}^{k}$ and $\mathcal{Y}_{n, \varepsilon}^{k}$ such that

$$
\begin{array}{ll} 
& \left(a_{n, \varepsilon}^{k}, D_{(x, p)} \Theta_{n, \varepsilon}^{k}\left(z_{n, \varepsilon}^{k}\right), \mathcal{X}_{n, \varepsilon}^{k}\right) \in \overline{\mathcal{P}}^{+} \tilde{V}_{2}\left(t_{n, \varepsilon}^{k}, x_{n, \varepsilon}^{k}, p_{n, \varepsilon}^{k}\right) \\
\text { and } \quad\left(-b_{n, \varepsilon}^{k},-D_{(y, q)} \Theta_{n, \varepsilon}^{k}\left(z_{n, \varepsilon}^{k}\right), \mathcal{Y}_{n, \varepsilon}^{k}\right) \in \overline{\mathcal{P}}^{-} \tilde{V}_{1}\left(t_{n, \varepsilon}^{k}, y_{n, \varepsilon}^{k}, q_{n, \varepsilon}^{k}\right),
\end{array}
$$

see $[8]$ for the standard notations $\overline{\mathcal{P}}^{+}$and $\overline{\mathcal{P}}^{-}$, where

$$
\begin{aligned}
D_{x} \Theta_{n, \varepsilon}^{k}\left(z_{n, \varepsilon}^{k}\right) & =n^{2}\left(x_{n, \varepsilon}^{k}-y_{n, \varepsilon}^{k}\right)+D_{x} f_{\varepsilon}\left(x_{n, \varepsilon}^{k}\right), D_{p} \Theta_{n, \varepsilon}^{k}\left(z_{n, \varepsilon}^{k}\right)=k^{2}\left(p_{n, \varepsilon}^{k}-q_{n, \varepsilon}^{k}\right) \\
-D_{y} \Theta_{n, \varepsilon}^{k}\left(z_{n, \varepsilon}^{k}\right) & =n^{2}\left(x_{n, \varepsilon}^{k}-y_{n, \varepsilon}^{k}\right),-D_{q} \Theta_{n, \varepsilon}^{k}\left(z_{n, \varepsilon}^{k}\right)=k^{2}\left(p_{n, \varepsilon}^{k}-q_{n, \varepsilon}^{k}\right)
\end{aligned}
$$

and $a_{n, \varepsilon}^{k}, b_{n, \varepsilon}^{k}, \mathcal{X}_{n, \varepsilon}^{k}$ and $\mathcal{Y}_{n, \varepsilon}^{k}$ satisfy

$$
\left\{\begin{array}{c}
a_{n, \varepsilon}^{k}+b_{n, \varepsilon}^{k}=0 \\
\left(\begin{array}{cc}
\mathcal{X}_{n, \varepsilon}^{k} & 0 \\
0 & -\mathcal{Y}_{n, \varepsilon}^{k}
\end{array}\right) \leq A_{n, \varepsilon}^{k}+\eta\left(A_{n, \varepsilon}^{k}\right)^{2} .
\end{array}\right.
$$

with

$$
A_{n, \varepsilon}^{k}:=\left(\begin{array}{cccc}
n^{2} I_{d}+D_{x}^{2} f_{\varepsilon}\left(x_{n, \varepsilon}^{k}\right) & 0 & -n^{2} I_{d} & 0 \\
0 & k^{2} I_{\kappa} & 0 & -k^{2} I_{\kappa} \\
-n^{2} I_{d} & 0 & n^{2} I_{d} & 0 \\
0 & -k^{2} I_{\kappa} & 0 & k^{2} I_{\kappa}
\end{array}\right),
$$

where $I_{d}$ and $I_{\kappa}$ stand for the $d \times d$ and $\kappa \times \kappa$ identity matrices.

We now study different cases:

Case 1. If, up to a subsequence, $M_{I J}^{\lambda}\left(V_{1}\left(t_{n, \varepsilon}^{k}, y_{n, \varepsilon}^{k}, q_{n, \varepsilon}^{k}\right), \psi_{1}\left(t_{n, \varepsilon}^{k}, y_{n, \varepsilon}^{k}, q_{n, \varepsilon}^{k}\right),-e^{-\rho t_{n, \varepsilon}^{k}} D_{q} \Theta_{n, \varepsilon}^{k}\left(z_{n, \varepsilon}^{k}\right)\right) \geq 0$, then there exists $\iota_{n, \varepsilon}^{k} \geq 0$ such that

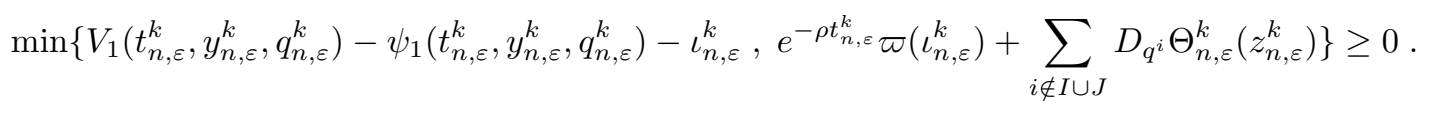

If, up to a subsequence, $V_{2}\left(t_{n, \varepsilon}^{k}, x_{n, \varepsilon}^{k}, p_{n, \varepsilon}^{k}\right)-\psi_{2}\left(t_{n, \varepsilon}^{k}, x_{n, \varepsilon}^{k}, p_{n, \varepsilon}^{k}\right) \leq \iota_{n, \varepsilon}^{k}$, we obtain a contradiction by using (4.38), the fact that $\psi_{1} \geq \psi_{2}, \psi_{1}$ and $-\psi_{2}$ are lower-semicontinuous, and by (4.30), (4.31), (4.32) and (4.34).

We then assume that

$$
V_{2}\left(t_{n, \varepsilon}^{k}, x_{n, \varepsilon}^{k}, p_{n, \varepsilon}^{k}\right)-\psi_{2}\left(t_{n, \varepsilon}^{k}, x_{n, \varepsilon}^{k}, p_{n, \varepsilon}^{k}\right)>\iota_{n, \varepsilon}^{k} .
$$

Then, there exists $\tilde{\iota}_{n, \varepsilon}^{k}>\iota_{n, \varepsilon}^{k}$ satisfying

$$
V_{2}\left(t_{n, \varepsilon}^{k}, x_{n, \varepsilon}^{k}, p_{n, \varepsilon}^{k}\right)-\psi_{2}\left(t_{n, \varepsilon}^{k}, x_{n, \varepsilon}^{k}, p_{n, \varepsilon}^{k}\right)>\tilde{\iota}_{n, \varepsilon}^{k}
$$


so that, by the subsolution property of $V_{2}$,

$$
e^{\rho t_{n, \varepsilon}^{k} \varpi\left(\tilde{\iota}_{n, \varepsilon}^{k}\right)}-\sum_{i \notin I \cup J} D_{p^{i}} \Theta_{n, \varepsilon}^{k}\left(z_{n, \varepsilon}^{k}\right) \leq 0 .
$$

Since $D_{p^{i}} \Theta_{n, \varepsilon}^{k}\left(z_{n, \varepsilon}^{k}\right)=-D_{q^{i}} \Theta_{n, \varepsilon}^{k}\left(z_{n, \varepsilon}^{k}\right)$ by (4.35) and (4.36), (4.38) implies that $\varpi\left(\tilde{l}_{n, \varepsilon}^{k}\right) \leq \varpi\left(\iota_{n, \varepsilon}^{k}\right)$. Since $\tilde{\iota}_{n, \varepsilon}^{k}>\iota_{n, \varepsilon}^{k}$ and $\varpi$ is strictly increasing, recall (3.9), this leads to a contradiction.

From now on, we assume that

$$
M_{I J}^{\lambda}\left(V_{1}\left(t_{n, \varepsilon}^{k}, y_{n, \varepsilon}^{k}, q_{n, \varepsilon}^{k}\right), \psi_{1}\left(t_{n, \varepsilon}^{k}, y_{n, \varepsilon}^{k}, q_{n, \varepsilon}^{k}\right),-e^{-\rho t_{n, \varepsilon}^{k}} D_{q} \Theta_{n, \varepsilon}^{k}\left(z_{n, \varepsilon}^{k}\right)\right)<0 .
$$

Case 2. If, up to a subsequence,

$$
V_{2}\left(t_{n, \varepsilon}^{k}, x_{n, \varepsilon}^{k}, p_{n, \varepsilon}^{k}\right) \leq \ell \vee \psi_{2}\left(t_{n, \varepsilon}^{k}, x_{n, \varepsilon}^{k}, p_{n, \varepsilon}^{k}\right) .
$$

It follows from the supersolution property of $V_{1}$ that $V_{1}\left(t_{n, \varepsilon}^{k}, y_{n, \varepsilon}^{k}, q_{n, \varepsilon}^{k}\right) \geq \ell$. Since we also have $V_{1} \geq \psi_{2}$ by assumption, passing to the limit leads to a contradiction as above.

Case 3. From now on, we can therefore assume that $V_{2}\left(t_{n, \varepsilon}^{k}, x_{n, \varepsilon}^{k}, p_{n, \varepsilon}^{k}\right)>\ell \vee \psi_{2}\left(t_{n, \varepsilon}^{k}, x_{n, \varepsilon}^{k}, p_{n, \varepsilon}^{k}\right)$, holds. In particular, the subsolution property of $V_{2}$ and (4.35)-(4.36) imply that

$$
\sum_{i \notin I \cup J} D_{p^{i}} \Theta_{n, \varepsilon}^{k}\left(z_{n, \varepsilon}^{k}\right)=-\sum_{i \notin I \cup J} D_{q^{i}} \Theta_{n, \varepsilon}^{k}\left(z_{n, \varepsilon}^{k}\right) \geq \varpi\left(l_{n, \varepsilon}^{k}\right)>0
$$

where

$$
\bar{\iota}_{n, \varepsilon}^{k}:=\left(V_{2}-\psi_{2}\right)\left(t_{n, \varepsilon}^{k}, x_{n, \varepsilon}^{k}, p_{n, \varepsilon}^{k}\right) / 2>0
$$

For later use, note that

$$
\liminf _{\varepsilon \rightarrow 0} \liminf _{n \rightarrow \infty} \bar{\iota}_{n, \varepsilon}^{k}>0
$$

since otherwise, we would get a contradiction to (4.32) as above since $V_{1} \geq \psi_{2}$ by assumption. The inequality (4.40) implies that there must exist some $i_{n, \varepsilon}^{k} \notin I \cup J$ such that

$$
D_{p^{i k}, \varepsilon} \Theta_{n, \varepsilon}^{k}\left(z_{n, \varepsilon}^{k}\right)=-D_{q^{i k}{ }_{n, \varepsilon}^{k}} \Theta_{n, \varepsilon}^{k}\left(z_{n, \varepsilon}^{k}\right) \geq \varpi\left(\iota_{n, \varepsilon}^{k}\right) / \kappa>0
$$

recall (4.35)-(4.36). Let us now fix $\left(u_{n, \varepsilon}^{k}, \alpha_{n, \varepsilon}^{k}\right) \in U \times A_{I J}$ such that

$$
\left(u_{n, \varepsilon}^{k}, \alpha_{n, \varepsilon}^{k}\right) \in N_{I J}^{1 / n}\left(y_{n, \varepsilon}^{k},-e^{-\rho t_{n, \varepsilon}^{k}} D_{y} \Theta_{n, \varepsilon}^{k}\left(z_{n, \varepsilon}^{k}\right),-e^{-\rho t_{n, \varepsilon}^{k}} D_{q} \Theta_{n, \varepsilon}^{k}\left(z_{n, \varepsilon}^{k}\right)\right)
$$

i.e.

$$
\left(u_{n, \varepsilon}^{k}\right)^{\top} \sigma\left(y_{n, \varepsilon}^{k}\right)=-e^{-\rho t_{n, \varepsilon}^{k}} D_{y} \Theta_{n, \varepsilon}^{k}\left(z_{n, \varepsilon}^{k}\right)^{\top} \sigma_{X}\left(y_{n, \varepsilon}^{k}\right)-e^{-\rho t_{n, \varepsilon}^{k}} D_{q} \Theta_{n, \varepsilon}^{k}\left(z_{n, \varepsilon}^{k}\right)^{\top} \alpha_{n, \varepsilon}^{k}+\xi_{n, \varepsilon}^{k}
$$

for some

$$
\xi_{n, \varepsilon}^{k} \in \mathbb{R}^{d} \text { such that }\left|\xi_{n, \varepsilon}^{k}\right| \in\left[-n^{-1}, n^{-1}\right] .
$$

Using (4.35)-(4.36) and (4.42), we see that $\bar{\alpha}_{n, \varepsilon}^{k}$ defined as

$$
\left(\bar{\alpha}_{n, \varepsilon}^{k}\right)^{\cdot i}:=\left(\alpha_{n, \varepsilon}^{k}\right)^{\cdot i} \text { for } i \neq i_{n, \varepsilon}^{k}
$$


and

$$
\begin{aligned}
\left.D_{p^{i k, \varepsilon}} \Theta_{n, \varepsilon}^{k}\left(z_{n, \varepsilon}^{k}\right)\left(\bar{\alpha}_{n, \varepsilon}^{k}-\alpha_{n, \varepsilon}^{k}\right)\right)_{n, \varepsilon}^{\cdot k}:= & e^{\rho t_{n, \varepsilon}^{k}}\left(u_{n, \varepsilon}^{k}\right)^{\top}\left(\sigma\left(x_{n, \varepsilon}^{k}\right)-\sigma\left(y_{n, \varepsilon}^{k}\right)\right) \\
& -D_{x} \Theta_{n, \varepsilon}^{k}\left(z_{n, \varepsilon}^{k}\right)^{\top}\left(\sigma_{X}\left(x_{n, \varepsilon}^{k}\right)-\sigma_{X}\left(y_{n, \varepsilon}^{k}\right)\right) \\
& -D_{x} f_{\varepsilon}\left(x_{n, \varepsilon}^{k}\right)^{\top} \sigma_{X}\left(x_{n, \varepsilon}^{k}\right)+e^{\rho t_{n, \varepsilon}^{k}} \xi_{n, \varepsilon}^{k},
\end{aligned}
$$

satisfies $\left(u_{n, \varepsilon}^{k}, \bar{\alpha}_{n, \varepsilon}^{k}\right) \in N_{I J}^{0}\left(x_{n, \varepsilon}^{k}, e^{-\rho t_{n, \varepsilon}^{k}} D_{x} \Theta_{n, \varepsilon}^{k}\left(z_{n, \varepsilon}^{k}\right), e^{-\rho t_{n, \varepsilon}^{k}} D_{p} \Theta_{n, \varepsilon}^{k}\left(z_{n, \varepsilon}^{k}\right)\right)$.

Using the super- and subsolution properties of $V_{1}$ and $V_{2}$, we can then choose $\left(u_{n, \varepsilon}^{k}, \alpha_{n, \varepsilon}^{k}\right)$ such that

$$
\begin{aligned}
-\frac{1}{n} \leq & b_{n, \varepsilon}^{k}+\rho \tilde{V}_{1}\left(t_{n, \varepsilon}^{k}, y_{n, \varepsilon}^{k}, q_{n, \varepsilon}^{k}\right) \\
& +e^{\rho t_{n, \varepsilon}^{k}} \mu_{Y}\left(y_{n, \varepsilon}^{k}, u_{n, \varepsilon}^{k}\right)+\mu_{X}\left(y_{n, \varepsilon}^{k}\right)^{\top} D_{y} \Theta_{n, \varepsilon}^{k}\left(z_{n, \varepsilon}^{k}\right)-\frac{1}{2} \operatorname{Trace}\left[\sigma_{X, P} \sigma_{X, P}^{\top}\left(y_{n, \varepsilon}^{k}, \alpha_{n, \varepsilon}^{k}\right) \mathcal{Y}_{n, \varepsilon}^{k}\right],
\end{aligned}
$$

and

$$
\begin{aligned}
\frac{1}{n} \geq & -a_{n, \varepsilon}^{k}+\rho \tilde{V}_{2}\left(t_{n, \varepsilon}^{k}, x_{n, \varepsilon}^{k}, p_{n, \varepsilon}^{k}\right) \\
& +e^{\rho t_{n, \varepsilon}^{k}} \mu_{Y}\left(x_{n, \varepsilon}^{k}, u_{n, \varepsilon}^{k}\right)-\mu_{X}\left(x_{n, \varepsilon}^{k}\right)^{\top} D_{x} \Theta_{n, \varepsilon}^{k}\left(z_{n, \varepsilon}^{k}\right)-\frac{1}{2} \operatorname{Trace}\left[\sigma_{X, P} \sigma_{X, P}^{\top}\left(x_{n, \varepsilon}^{k}, \bar{\alpha}_{n, \varepsilon}^{k}\right) \mathcal{X}_{n, \varepsilon}^{k}\right] .
\end{aligned}
$$

Hence,

$$
\begin{aligned}
-\frac{2}{n} \leq & b_{n, \varepsilon}^{k}+a_{n, \varepsilon}^{k}-\rho\left(\tilde{V}_{2}\left(t_{n, \varepsilon}^{k}, x_{n, \varepsilon}^{k}, p_{n, \varepsilon}^{k}\right)-\tilde{V}_{1}\left(t_{n, \varepsilon}^{k}, y_{n, \varepsilon}^{k}, q_{n, \varepsilon}^{k}\right)\right) \\
& -e^{\rho t_{n, \varepsilon}^{k}}\left(\mu_{Y}\left(x_{n, \varepsilon}^{k}, u_{n, \varepsilon}^{k}\right)-\mu_{Y}\left(y_{n, \varepsilon}^{k}, u_{n, \varepsilon}^{k}\right)\right)+\mu_{X}\left(x_{n, \varepsilon}^{k}\right)^{\top} D_{x} \Theta_{n, \varepsilon}^{k}\left(z_{n, \varepsilon}^{k}\right)+\mu_{X}\left(y_{n, \varepsilon}^{k}\right)^{\top} D_{y} \Theta_{n, \varepsilon}^{k}\left(z_{n, \varepsilon}^{k}\right) \\
& +\frac{1}{2} \operatorname{Trace}\left[\sigma_{X, P} \sigma_{X, P}^{\top}\left(x_{n, \varepsilon}^{k}, \bar{\alpha}_{n, \varepsilon}^{k}\right) \mathcal{X}_{n, \varepsilon}^{k}-\sigma_{X, P} \sigma_{X, P}^{\top}\left(y_{n, \varepsilon}^{k}, \alpha_{n, \varepsilon}^{k}\right) \mathcal{Y}_{n, \varepsilon}^{k}\right] .
\end{aligned}
$$

Using (4.37), and then letting $\eta \rightarrow 0$,

$$
\begin{aligned}
-\frac{2}{n} \leq & -\rho\left(\tilde{V}_{2}\left(t_{n, \varepsilon}^{k}, x_{n, \varepsilon}^{k}, p_{n, \varepsilon}^{k}\right)-\tilde{V}_{1}\left(t_{n, \varepsilon}^{k}, y_{n, \varepsilon}^{k}, q_{n, \varepsilon}^{k}\right)\right) \\
& -e^{\rho t_{n, \varepsilon}^{k}} u_{n, \varepsilon}^{k \top}\left(\mu\left(x_{n, \varepsilon}^{k}\right)-\mu\left(y_{n, \varepsilon}^{k}\right)\right)+n^{2}\left(\mu_{X}^{\top}\left(x_{n, \varepsilon}^{k}\right)-\mu_{X}^{\top}\left(y_{n, \varepsilon}^{k}\right)\right)\left(x_{n, \varepsilon}^{k}-y_{n, \varepsilon}^{k}\right) \\
& +D_{x} f_{\varepsilon}\left(x_{\varepsilon}^{k}\right)^{\top} \mu_{X}\left(x_{\varepsilon}^{k}\right)+\frac{1}{2} \operatorname{Trace}\left[\sigma_{X}\left(x_{n, \varepsilon}^{k}\right) \sigma_{X}\left(x_{n, \varepsilon}^{k}\right)^{\top} D_{x}^{2} f_{\varepsilon}\left(x_{n, \varepsilon}^{k}\right)\right] \\
& +\frac{n^{2}}{2} \operatorname{Trace}\left[\left(\sigma_{X}\left(x_{n, \varepsilon}^{k}\right)-\sigma_{X}\left(y_{n, \varepsilon}^{k}\right)\right)\left(\sigma_{X}\left(x_{n, \varepsilon}^{k}\right)-\sigma_{X}\left(y_{n, \varepsilon}^{k}\right)\right)^{\top}\right] \\
& +\frac{k^{2}}{2}\left\|\alpha_{n, \varepsilon}^{k}-\bar{\alpha}_{n, \varepsilon}^{k}\right\|^{2} .
\end{aligned}
$$

We now send $n \rightarrow \infty$ and then $\varepsilon \rightarrow 0$ in the above inequality, and deduce from (4.30), (4.31), (4.33), (4.45),(4.32), (4.41), (4.42), (4.44) and the Lipschitz continuity of $\left(\mu_{X}, \sigma_{X}\right)$ that

$$
0 \leq-\rho m
$$

which contradicts the fact that $\rho, m>0$.

Part 2: We now consider the case $I \cup J=\mathcal{K}$. Part of the arguments being similar as in Part 1, we only sketch them.

Step 1. In the case $I \cup J=\mathcal{K}$, we can work as if $V_{1}$ and $V_{2}$ do not depend on $p$. Indeed, $a \in A_{I J}$ implies $a=0$, so that the derivatives in $p$ do not appear in the operator. Moreover, recalling the convention (3.10) and the discussion of Section 3.3.1, we see that a function $w$ is a viscosity supersolution (resp. subsolution) of (2.20) (resp. (2.21)) if and only if it is a viscosity supersolution (resp. subsolution) of

$$
\min \left\{\varphi-\ell,-\partial_{t} \varphi+\bar{F}_{I J}\left(\cdot, D \varphi, D^{2} \varphi\right) ; R(x, q)\right\}=0 \text { on } D_{I J}
$$


where

$$
\bar{F}_{I J}(x, q, Q):=L^{q^{\top} \operatorname{diag}[x], 0}(x, q, Q)
$$

and

$$
R(x, q):=\inf _{|\zeta| \leq 1}\left(\delta_{U}(\zeta)-\zeta^{\top} \operatorname{diag}[x] q\right) .
$$

Note that here we do not need to consider the semicontinuous envelopes of the operator since the unbounded control $a$ does not play any role and $U$ is bounded.

Given $\rho>0$, we set $\tilde{V}_{1}(t, x):=e^{\rho t} V_{1}(t, x)$ and $\tilde{V}_{2}(t, x):=e^{\rho t} V_{2}(t, x)$. We now choose $u \in \operatorname{int} U \cap$ $(-\infty, 0)^{d}$, which is possible since $0 \in \operatorname{int} U$ by assumption, $\delta \in(0,1)$ and define

$$
\tilde{V}_{\delta}:=(1-\delta) \tilde{V}_{1}+\delta \psi
$$

where, for some $\epsilon>0$,

$$
\psi(x):=\epsilon e^{\sum_{i \leq d} u^{i} x^{i}} .
$$

Note that, since $\operatorname{diag}[x] \psi(x)$ is bounded on $(0, \infty)^{d}, \operatorname{int} U$ is convex and contains 0 , we can choose $\epsilon>0$ small enough so that

$$
0<v \leq \delta_{U}(\zeta)-\zeta^{\top} e^{-\rho t} \operatorname{diag}[x] \psi(x) u=\delta_{U}(\zeta)-\zeta^{\top} e^{-\rho t} \operatorname{diag}[x] D_{x} \psi(x)
$$

where $v>0$ does not depend on $\zeta$ and $(t, x) \in[0, T] \times(0, \infty)^{d}$.

In order to show that $V_{2} \leq V_{1}$, we argue by contradiction. We therefore assume that

$$
\sup _{[0, T] \times(0, \infty)^{d}}\left(\tilde{V}_{2}-\tilde{V}_{\delta}\right)=: 2 m>0,
$$

for $\delta$ small enough, and work towards a contradiction.

Using the boundedness of $\tilde{V}_{2}$ and $\tilde{V}_{\delta}$, and (4.48), we deduce that

$$
\Phi^{\varepsilon}:=\tilde{V}_{2}-\tilde{V}_{\delta}-f_{\varepsilon},
$$

where $f_{\varepsilon}$ is defined as in Part 1 , admits a maximum $\left(t_{\varepsilon}, x_{\varepsilon}\right)$ on $[0, T] \times(0, \infty)^{d}$, which, for $\varepsilon>0$ small enough, satisfies

$$
\Phi^{\varepsilon}\left(t_{\varepsilon}, x_{\varepsilon}\right) \geq m>0 .
$$

Without loss of generality, we can choose $\left(x_{\varepsilon}\right)_{\varepsilon>0}$ such that

$$
f_{\varepsilon}\left(x_{\varepsilon}\right) \underset{\varepsilon \rightarrow 0}{\longrightarrow} 0
$$

which implies (see Part 1)

$$
\left|D_{x} f_{\varepsilon}\left(x_{\varepsilon}\right)^{\top} \operatorname{diag}\left[x_{\varepsilon}\right]\right|+\left|D_{x} f_{\varepsilon}\left(x_{\varepsilon}\right)^{\top} \mu_{X}\left(x_{\varepsilon}\right)\right|+\left|D_{x} f_{\varepsilon}\left(x_{\varepsilon}\right)^{\top} \sigma_{X}\left(x_{\varepsilon}\right)\right|+\left|\operatorname{Trace}\left[\sigma_{X} \sigma_{X}^{\top}\left(x_{\varepsilon}\right) D_{x}^{2} f_{\varepsilon}\left(x_{\varepsilon}\right)\right]\right| \underset{\varepsilon \rightarrow 0}{\longrightarrow} 0 .
$$

For $n \geq 1$, we then define the function $\Psi_{n}^{\varepsilon}$ on $[0, T] \times(0, \infty)^{2 d}$ by

$$
\Psi_{n}^{\varepsilon}(t, x, y):=\tilde{V}_{2}(t, x)-\tilde{V}_{\delta}(t, y)-f_{\varepsilon}(x)-\frac{n^{2}}{2}|x-y|^{2} .
$$

It follows again from the boundedness of $\tilde{V}_{2}$ and $\tilde{V}_{\delta}$ that $\Psi_{n}^{\varepsilon}$ attains its maximum at some $\left(t_{n}^{\varepsilon}, x_{n}^{\varepsilon}, y_{n}^{\varepsilon}\right)$ $\in[0, T] \times(0, \infty)^{2 d}$. Moreover, the same arguments as in Part 1 imply that, up to a subsequence and after possibly changing $\left(t_{\varepsilon}, x_{\varepsilon}\right)_{\varepsilon>0}$,

$$
x_{n}^{\varepsilon}, y_{n}^{\varepsilon} \underset{n \rightarrow \infty}{\longrightarrow} x_{\varepsilon} \in(0, \infty)^{d}, t_{n}^{\varepsilon} \underset{n \rightarrow \infty}{\longrightarrow} t_{\varepsilon},
$$


and

$$
\begin{gathered}
n^{2}\left|x_{n}^{\varepsilon}-y_{n}^{\varepsilon}\right|^{2} \underset{n \rightarrow \infty}{\longrightarrow} 0, \\
\tilde{V}_{2}\left(t_{n}^{\varepsilon}, x_{n}^{\varepsilon}\right)-\tilde{V}_{\delta}\left(t_{n}^{\varepsilon}, y_{n}^{\varepsilon}\right) \underset{n \rightarrow \infty}{\longrightarrow}\left(\tilde{V}-\tilde{V}_{\delta}\right)\left(t_{\varepsilon}, x_{\varepsilon}\right) \geq m+f_{\varepsilon}\left(x_{\varepsilon}\right)>0 .
\end{gathered}
$$

By similar arguments as in Part 1, we can not have $t_{n}^{\varepsilon}=T$, up to a subsequence. We can then assume from now on that $t_{n}^{\varepsilon}<T$ for all $\varepsilon, n$.

Step 2. Since $t_{n}^{\varepsilon}<T$ and $\left(x_{n}^{\varepsilon}, y_{n}^{\varepsilon}\right) \in(0, \infty)^{2 d}$, see (4.52), we can appeal to Ishii's Lemma to deduce that, for each $\eta>0$, there are real coefficients $b_{1, n}^{\varepsilon}, b_{2, n}^{\varepsilon}$ and symmetric matrices $\mathcal{X}_{n}^{\varepsilon, \eta}$ and $\mathcal{Y}_{n}^{\varepsilon, \eta}$ such that

$$
\left(b_{1, n}^{\varepsilon}, p_{n}^{\varepsilon}, \mathcal{X}_{n}^{\varepsilon, \eta}\right) \in \overline{\mathcal{P}}^{+} \tilde{V}_{2}\left(t_{n}^{\varepsilon}, x_{n}^{\varepsilon}\right) \quad \text { and } \quad\left(-b_{2, n}^{\varepsilon}, q_{n}^{\varepsilon}, \mathcal{Y}_{n}^{\varepsilon, \eta}\right) \in \overline{\mathcal{P}}^{-} \tilde{V}_{\delta}\left(t_{n}^{\varepsilon}, y_{n}^{\varepsilon}\right)
$$

where

$$
p_{n}^{\varepsilon}:=n^{2}\left(x_{n}^{\varepsilon}-y_{n}^{\varepsilon}\right)+D f_{\varepsilon}\left(x_{n}^{\varepsilon}\right), q_{n}^{\varepsilon}:=n^{2}\left(x_{n}^{\varepsilon}-y_{n}^{\varepsilon}\right),
$$

and $b_{1, n}^{\varepsilon}, b_{2, n}^{\varepsilon}, \mathcal{X}_{n}^{\varepsilon, \eta}$ and $\mathcal{Y}_{n}^{\varepsilon, \eta}$ satisfy

$$
\left\{\begin{array}{cc}
b_{1, n}^{\varepsilon}+b_{2, n}^{\varepsilon} & =0 \\
\left(\begin{array}{cc}
\mathcal{X}_{n}^{\varepsilon, \eta} & 0 \\
0 & -\mathcal{Y}_{n}^{\varepsilon, \eta}
\end{array}\right)^{\varepsilon} \leq A_{n}^{\varepsilon}+\eta\left(A_{n}^{\varepsilon}\right)^{2}
\end{array}\right.
$$

with

$$
A_{n}^{\varepsilon}:=\left(\begin{array}{cc}
2 n^{2} I_{d}+D^{2} f_{\varepsilon}\left(x_{n}^{\varepsilon}\right) & -2 n^{2} I_{d} \\
-2 n^{2} I_{d} & 2 n^{2} I_{d}
\end{array}\right)
$$

We now study in different cases:

Case 1. If, up to a subsequence, $\tilde{V}_{2}\left(t_{n}^{\varepsilon}, x_{n}^{\varepsilon}\right) \leq \ell e^{\rho t_{n}^{\varepsilon}}$, then we get a contradiction for $n$ large and $\delta$ small, since $\tilde{V}_{1}\left(t_{n}^{\varepsilon}, y_{n}^{\varepsilon}\right) \geq \ell e^{\rho t_{n}^{\varepsilon}}$ and $\psi \geq 0 \geq \ell e^{\rho t_{n}^{\varepsilon}}$.

Case 2. If, up to a subsequence, $R\left(x_{n}^{\varepsilon}, e^{-\rho t_{n}^{\varepsilon}} p_{n}^{\varepsilon}\right)>0$, then we must have

$$
\rho \tilde{V}_{2}\left(t_{n}^{\varepsilon}, x_{n}^{\varepsilon}\right)-b_{1, n}^{\varepsilon}+\bar{F}_{I J}\left(x_{n}^{\varepsilon}, p_{n}^{\varepsilon}, \mathcal{X}_{n}^{\varepsilon, \eta}\right) \leq 0 .
$$

Since $\psi, x \in(0, \infty)^{d} \mapsto\left(\operatorname{diag}[x] D_{x} \psi(x), \operatorname{diag}[x]^{2} D_{x}^{2} \psi(x)\right)$ and $(\mu, \sigma)$ are bounded, one easily checks that the supersolution property of $V_{1}$ implies that

$$
\rho \tilde{V}_{\delta}\left(t_{n}^{\varepsilon}, y_{n}^{\varepsilon}\right)+b_{2, n}^{\varepsilon}+\bar{F}_{I J}\left(y_{n}^{\varepsilon}, q_{n}^{\varepsilon}, \mathcal{Y}_{n}^{\varepsilon, \eta}\right) \geq O(\delta) .
$$

Standard arguments based on (4.51), (4.52), (4.53), (4.54) and (4.56) then leads to a contradiction for $\delta>0$ small enough, after sending $\eta \rightarrow 0, n \rightarrow \infty$ and then $\varepsilon \rightarrow 0$.

Case 3. We can now assume that $R\left(x_{n}^{\varepsilon}, e^{-\rho t_{n}^{\varepsilon}} p_{n}^{\varepsilon}\right) \leq 0$. By the supersolution property of $V_{1}$, we have

$$
R\left(y_{n}^{\varepsilon}, e^{-\rho t_{n}^{\varepsilon}} \frac{q_{n}^{\varepsilon}-\delta D_{y} \psi\left(y_{n}^{\varepsilon}\right)}{1-\delta}\right) \geq 0
$$

We can then find $\zeta_{n}^{\varepsilon}$ such that $\left|\zeta_{n}^{\varepsilon}\right|=1$ and

$$
\begin{aligned}
0 \geq & \left(e^{\rho t_{n}^{\varepsilon}} \delta_{U}\left(\zeta_{n}^{\varepsilon}\right)-\left(\zeta_{n}^{\varepsilon}\right)^{\top} \operatorname{diag}\left[x_{n}^{\varepsilon}\right] p_{n}^{\varepsilon}-e^{\rho t_{n}^{\varepsilon}} \delta_{U}\left(\zeta_{n}^{\varepsilon}\right)+\left(\zeta_{n}^{\varepsilon}\right)^{\top} \operatorname{diag}\left[y_{n}^{\varepsilon}\right] q_{n}^{\varepsilon}\right) \\
& +\delta e^{\rho t_{n}^{\varepsilon}}\left(\delta_{U}\left(\zeta_{n}^{\varepsilon}\right)-\left(\zeta_{n}^{\varepsilon}\right)^{\top} e^{-\rho t_{n}^{\varepsilon}} \operatorname{diag}\left[y_{n}^{\varepsilon}\right] D_{y} \psi\left(y_{n}^{\varepsilon}\right)\right) .
\end{aligned}
$$

In view of (4.47) and (4.55), this implies that

$\left(\zeta_{n}^{\varepsilon}\right)^{\top}\left(\operatorname{diag}\left[x_{n}^{\varepsilon}-y_{n}^{\varepsilon}\right] n^{2}\left(x_{n}^{\varepsilon}-y_{n}^{\varepsilon}\right)+\operatorname{diag}\left[x_{n}^{\varepsilon}\right] D_{x} f_{\varepsilon}\left(x_{n}^{\varepsilon}\right)\right)=\left(\zeta_{n}^{\varepsilon}\right)^{\top}\left(\operatorname{diag}\left[x_{n}^{\varepsilon}\right] p_{n}^{\varepsilon}-\operatorname{diag}\left[y_{n}^{\varepsilon}\right] q_{n}^{\varepsilon}\right) \geq v \delta e^{\rho t_{n}^{\varepsilon}}$.

Using (4.51), (4.52), (4.53) and (4.55), this leads to a contradiction as $n \rightarrow \infty$ and then $\varepsilon \rightarrow 0$. 


\subsubsection{Additional technical results for the convergence of the finite differences scheme}

We start with a simple remark concerning the PDEs obtained in the interior of the domains in Section 3.3.3.

Remark 4.1 (i) The result of Proposition 4.6 still holds if $V_{1}$ is a supersolution on $\bar{D}_{I J}$ of

$$
(\varphi-w) \wedge \max \left\{\varphi-\omega, H_{I J}^{\lambda *}\left[\varphi, \psi_{1}\right]\right\}=0 \text { on } D_{I J}
$$

and $V_{2}$ is a subsolution on $\bar{D}_{I J}$ of

$$
(\varphi-w) \wedge \max \left\{\varphi-\omega, H_{I J *}^{\lambda}\left[\varphi, \psi_{2}\right]\right\}=0 \text { on } D_{I J},
$$

for some continuous map $w, \omega$. In this case, the proof of Proposition 4.6 can be easily modified by studying simple additional cases. In the case where $w \geq \psi_{2}=\psi_{1}$, the assumption $V_{1} \geq \psi_{2}$ on $D_{I J}$ in not necessary anymore in Proposition 4.6, since it is induced by the super-solution property of $V_{1}$.

(ii) Obviously, the result of Proposition 4.6 still holds if $V_{1}$ is a supersolution on $\bar{D}_{I J}$ of

$$
\max \left\{\varphi-\omega, H_{I J}^{\lambda *}\left[\varphi, \psi_{1}\right]\right\}=0 \text { on } D_{I J}
$$

and $V_{2}$ is a subsolution on $\bar{D}_{I J}$ of

$$
\max \left\{\varphi-\omega, H_{I J *}^{\lambda}\left[\varphi, \psi_{2}\right]\right\}=0 \text { on } D_{I J},
$$

for some continuous map $\omega$.

(iii) Note that in the two above cases, we can replace $H_{I J}^{\lambda *}$ by $H_{I J}^{\bar{a} *}$ defined as $H_{I J}^{\lambda *}$ but with $A_{I J}^{\bar{a}}$ instead of $A_{I J}, \bar{a}>0$. The proof follows line by line the one of Proposition 4.6, up to the study of simple additional cases as mentioned in (i) and (ii) above.

We now discuss the boundary condition as $t \rightarrow T$.

Lemma 4.1 Fix $(I, J) \in \mathcal{P}_{\kappa}$.

(i) Let $V_{1}$ be a supersolution on $\bar{D}_{I J}$ of

$$
\begin{gathered}
\max \left\{\varphi-L, \min \left\{\varphi-\ell,-\partial_{t} \varphi+F_{I J}^{\bar{a} *}\left(\cdot, D \varphi, D^{2} \varphi\right)\right\}, M_{I J}^{\lambda}\left(\varphi, v_{J^{c} J}^{\lambda}, D_{p} \varphi\right), \varphi-G^{\lambda}\right\}=0 \\
\text { on }\{T\} \times(0, \infty)^{d} \times \bar{B}_{I J} .
\end{gathered}
$$

Then, $V_{1}$ is a supersolution on $\bar{D}_{I J}$ of

$$
\max \left\{M_{I J}^{\lambda}\left(\varphi, v_{J^{c} J}^{\lambda}, D_{p} \varphi\right), \varphi-G^{\lambda}\right\}=0 \quad \text { on }\{T\} \times(0, \infty)^{d} \times \bar{B}_{I J} .
$$

(ii) Let $V_{2}$ be a subsolution on $\bar{D}_{I J}$ of

$$
\begin{gathered}
\min \left\{\max \left\{\varphi-L, \min \left\{\varphi-\ell,-\partial_{t} \varphi+F_{I J *}\left(\cdot, D \varphi, D^{2} \varphi\right)\right\}, M_{I J}^{\lambda}\left(\varphi, v_{J^{c} J}^{\lambda}, D_{p} \varphi\right)\right\}, \varphi-G^{\lambda}\right\}=0 \\
\text { on }\{T\} \times(0, \infty)^{d} \times \bar{B}_{I J} .
\end{gathered}
$$

Then, $V_{2} \leq G^{\lambda}$ on $\{T\} \times(0, \infty)^{d} \times \bar{B}_{I J}$.

Proof. The proof is standard. We start with item (i). Given a test function $\varphi$ such that $\left(T, x_{0}, p_{0}\right) \in$ $\{T\} \times(0, \infty)^{d} \times \bar{B}_{I J}$ achieves a minimum of $V_{1}-\varphi$ on $\bar{D}_{I J}$, we define $\varphi_{n}(t, x, p):=\varphi(t, x, p)-n(T-t)$, $n \geq 1$. We assume that

$$
\max \left\{\varphi-L, M_{I J}^{\lambda}\left(\varphi, v_{J^{c} J}^{\lambda}, D_{p} \varphi\right), \varphi-G^{\lambda}\right\}\left(T, x_{0}, p_{0}\right)<0 .
$$


Since $\left(T, x_{0}, p_{0}\right)$ also achieves a minimum of $V_{1}-\varphi_{n}$, the supersolution property of $V_{1}$ implies that

$$
\min \left\{\varphi-\ell,-n-\partial_{t} \varphi+F_{I J}^{\bar{a} *}\left(\cdot, D \varphi, D^{2} \varphi\right)\right\}\left(T, x_{0}, p_{0}\right) \geq 0 .
$$

Sending $n \rightarrow \infty$, we obtain a contradiction since $F_{I J}^{\bar{a} *}\left(\cdot, D \varphi, D^{2} \varphi\right)\left(T, x_{0}, p_{0}\right)<\infty$, recall that $U$ and $A_{I J}^{\bar{a}}$ are bounded. We finally use the fact that $G^{\lambda} \leq L$, since $g \leq L$ by assumption.

We now discuss item (ii). By similar arguments as above, we first obtain that $V_{2}$ is a subsolution on $\bar{D}_{I J}$ of

$$
\min \left\{\max \left\{\varphi-L, \varphi-\ell, M_{I J}^{\lambda}\left(\varphi, v_{J^{c} J}^{\lambda}, D_{p} \varphi\right)\right\}, \varphi-G^{\lambda}\right\}=0 \text { on }\{T\} \times(0, \infty)^{d} \times \bar{B}_{I J} .
$$

We conclude by using the fact that $G^{\lambda} \geq \ell$.

Lemma 4.2 Let $V_{1}$ and $V_{2}$ be as in Lemma 4.1 for some $(I, J) \in \mathcal{P}_{\kappa}$. Assume that they take values in $[\ell, L]$. Then, $G^{\lambda} \geq V_{2}$ on $\{T\} \times(0, \infty)^{d} \times \bar{B}_{I J}$. If in addition $(I, J) \in \mathcal{P}_{\kappa}^{\kappa}$, then $G^{\lambda} \leq V_{1}$ on $\{T\} \times(0, \infty)^{d} \times \bar{B}_{I J}$.

Proof. The fact that $V_{2} \leq G^{\lambda}$ on $\{T\} \times(0, \infty)^{d} \times \bar{B}_{I J}$ follows from Lemma 4.1. We now show that $V_{1} \geq v_{I J}^{\lambda}$ on $\{T\} \times(0, \infty)^{d} \times \bar{B}_{I J}$. To see this, note that $V_{1}(T, x, p) \geq G^{\lambda}(x, p)$ implies $V_{1}(T, x, p) \geq$ $v_{I J}^{\lambda}(T, x, p)$ by Theorem 3.3. Recalling the convention (3.10), this concludes the proof for $(I, J) \in \mathcal{P}_{\kappa}^{\kappa}$.

We finally discuss the boundary condition as $p \rightarrow \partial B_{I J}$.

Lemma 4.3 Fix $(I, J) \in \mathcal{P}_{\kappa} \backslash \mathcal{P}_{\kappa}^{\kappa}$ and $\left(I^{\prime}, J^{\prime}\right) \supsetneq(I, J)$.

(i) Let $V_{1}$ be a bounded supersolution on $\bar{D}_{I J}$ of

$$
\begin{aligned}
& \max \left\{\left(\varphi-v_{J^{c} J}^{\lambda}\right) \wedge \max \left\{\varphi-L, \bar{H}_{I J}^{\bar{a} *}\left[\varphi, v_{J^{c} J}^{\lambda}\right]\right\}, \varphi-v_{I^{\prime} J^{\prime}}^{\lambda}\right\}=0 \quad \text { on } \quad \partial D_{I J} \cap D_{I^{\prime} J^{\prime}} \\
& \max \left\{\left(\varphi-v_{J^{c} J}^{\lambda}\right) \wedge \max \left\{\varphi-L, \bar{H}_{I J}^{\bar{a} *}\left[\varphi, v_{J^{c} J}^{\lambda}\right]\right\}, \varphi-G^{\lambda}\right\}=0 \quad \text { on } \quad\{T\} \times(0, \infty)^{d} \times\left(\bar{B}_{I J} \cap B_{I^{\prime} J^{\prime}}\right) .
\end{aligned}
$$

Then, $V_{1}$ is a bounded supersolution on $\bar{D}_{I^{\prime} J^{\prime}}$ of

$$
\begin{aligned}
& \max \left\{\left(\varphi-v_{J^{c} J}^{\lambda}\right) \wedge \max \left\{\varphi-L, \bar{H}_{I J^{\prime}}^{\bar{a} *}\left[\varphi, v_{J^{\prime c} J^{\prime}}^{\lambda}\right]\right\}, \varphi-v_{I^{\prime} J^{\prime}}^{\lambda}\right\}=0 \quad \text { on } \quad D_{I^{\prime} J^{\prime}} \\
& \varphi-G^{\lambda}=0 \quad \text { on } \quad\{T\} \times(0, \infty)^{d} \times B_{I^{\prime} J^{\prime}},
\end{aligned}
$$

(ii) Let $V_{2}$ be a bounded subsolution on $\bar{D}_{I J}$ of

$$
\begin{aligned}
& \min \left\{\varphi-v_{J^{c} J}^{\lambda}, \max \left\{\varphi-L, \bar{H}_{I J *}^{\lambda}\left[\varphi, v_{J^{c} J}^{\lambda}\right]\right\}, \varphi-v_{I^{\prime} J^{\prime}}^{\lambda}\right\}=0 \quad \text { on } \quad \partial D_{I J} \cap D_{I^{\prime} J^{\prime}} \\
& \min \left\{\varphi-v_{J^{c} J}^{\lambda}, \max \left\{\varphi-L, \bar{H}_{I J *}^{\lambda}\left[\varphi, v_{J^{c} J}^{\lambda}\right]\right\}, \varphi-G^{\lambda}\right\}=0 \quad \text { on } \quad\{T\} \times(0, \infty)^{d} \times\left(\bar{B}_{I J} \cap B_{I^{\prime} J^{\prime}}\right) . \\
& \text { Then, } V_{2} \leq v_{I^{\prime} J^{\prime}}^{\lambda} \text { on } D_{I^{\prime} J^{\prime}} \text { and } V_{2} \leq G^{\lambda} \text { on }\{T\} \times(0, \infty)^{d} \times B_{I^{\prime} J^{\prime}} .
\end{aligned}
$$

Proof. (i) It follows from the same argument as in the proof of Lemma 4.1 that $V_{1}$ is a bounded supersolution on $\bar{D}_{I J}$ of

$$
\begin{aligned}
& \max \left\{\left(\varphi-v_{J^{c} J}^{\lambda}\right) \wedge \max \left\{\varphi-L, \bar{H}_{I J}^{\bar{a} *}\left[\varphi, v_{J^{c} J}^{\lambda}\right]\right\}, \varphi-v_{I^{\prime} J^{\prime}}^{\lambda}\right\}=0 \quad \text { on } \quad \bar{D}_{I J} \cap D_{I^{\prime} J^{\prime}}, \\
& \max \left\{\left(\varphi-v_{I J}^{\lambda}\right) \wedge M_{I J}^{\lambda}\left[\varphi, v_{J^{c} J}^{\lambda}\right], \varphi-G^{\lambda}\right\}=0 \quad \text { on } \quad\{T\} \times(0, \infty)^{d} \times\left(\bar{B}_{I J} \cap B_{I^{\prime} J^{\prime}}\right) .
\end{aligned}
$$

By following, up to minor modifications (related to the fact that their test function has a derivative in $p$ that converges to $\infty$, see also Step 2. of the proof of Proposition 4.2 for an adaptation to our context), the arguments used in Section 6.1 of [6], we deduce that $V_{1}$ is a bounded supersolution on $\bar{D}_{I J}$ of

$$
\begin{gathered}
\max \left\{\left(\varphi-v_{J^{c} J}^{\lambda}\right) \wedge \max \left\{\varphi-L,-\partial_{t} \varphi+\bar{F}_{I J^{\prime}}^{\bar{a} *}\left(\cdot, D \varphi, D^{2} \varphi\right)\right\}, \varphi-v_{I^{\prime} J^{\prime}}^{\lambda}\right\}=0 \text { on } \bar{D}_{I J} \cap D_{I^{\prime} J^{\prime}}, \\
\varphi-G^{\lambda}=0 \text { on }\{T\} \times(0, \infty)^{d} \times\left(\bar{B}_{I J} \cap B_{I^{\prime} J^{\prime}}\right) .
\end{gathered}
$$


We conclude by using the fact that $-\partial_{t} \varphi+\bar{F}_{I J^{\prime}}^{\bar{a} *}\left(\cdot, D \varphi, D^{2} \varphi\right) \leq \bar{H}_{I J^{\prime}}^{\bar{a} *}\left[\varphi, v_{J^{\prime} c J^{\prime}}^{\lambda}\right]$.

(ii) Since $v_{J^{c} J}^{\lambda} \leq v_{I^{\prime} J^{\prime}}^{\lambda}$ on $\partial D_{I J} \cap D_{I^{\prime} J^{\prime}}$ and $v_{J^{c} J}^{\lambda} \leq G^{\lambda}$ on $\{T\} \times(0, \infty)^{d} \times\left(\bar{B}_{I J} \cap B_{I^{\prime} J^{\prime}}\right)$, see Theorem

3.3 and recall that $v^{\lambda}$ is non-decreasing in $p, V_{2}$ is indeed a bounded subsolution on $\bar{D}_{I J}$ of

$$
\begin{aligned}
& \min \left\{\max \left\{\varphi-L, \bar{H}_{I J *}^{\lambda}\left[\varphi, v_{J^{c} J}^{\lambda}\right]\right\}, \varphi-v_{I^{\prime} J^{\prime}}^{\lambda}\right\}=0 \quad \text { on } \quad \partial D_{I J} \cap D_{I^{\prime} J^{\prime}}, \\
& \min \left\{\max \left\{\varphi-L, \bar{H}_{I J *}^{\lambda}\left[\varphi, v_{J^{c} J}^{\lambda}\right]\right\}, \varphi-G^{\lambda}\right\}=0 \quad \text { on } \quad\{T\} \times(0, \infty)^{d} \times\left(\bar{B}_{I J} \cap B_{I^{\prime} J^{\prime}}\right) \text {. }
\end{aligned}
$$

By the same argument as in the proof of Lemma 4.1, we then deduce that $V_{2}$ is a bounded subsolution on $\bar{D}_{I J}$ of

$$
\begin{array}{rlll}
\min \left\{\max \left\{\varphi-L, \bar{H}_{I J}^{\lambda *}\left[\varphi, v_{J^{c} J}^{\lambda}\right]\right\}, \varphi-v_{I^{\prime} J^{\prime}}^{\lambda}\right\}=0 & \text { on } & D_{I^{\prime} J^{\prime}}, \\
\varphi-G^{\lambda}=0 & \text { on } & \{T\} \times(0, \infty)^{d} \times B_{I^{\prime} J^{\prime}} .
\end{array}
$$

We then argue as in Step 2. of the proof of Proposition 4.2 above to deduce that $V_{2} \leq v_{I^{\prime} J^{\prime}}^{\lambda}$ on $D_{I^{\prime} J^{\prime}}$.

\subsection{Proof of Theorem 3.2}

In order to complete the proof of Theorem 3.2, we first show that $v^{\lambda}$ is continuous on $\bar{D}$.

Proposition 4.7 The function $v^{\lambda}$ is continuous on $\bar{D}$.

Proof. We argue by induction.

Step 1. We first notice that $v_{I J}^{\lambda}$ is continuous on $\bar{D}$ for $(I, J) \in \mathcal{P}_{\kappa}^{\kappa}$. This is a direct consequence of Theorem 3.1 and Proposition 4.6.

Step 2. We now assume that $v_{I J}^{\lambda}$ is continuous on $\bar{D}_{I J}$ if $(I, J) \in \mathcal{P}_{\kappa}^{\kappa-k}$ for some $1 \leq k<\kappa$, and show that this implies that it holds for $(I, J) \in \mathcal{P}_{\kappa}^{\kappa-k-1}$.

By Step 1, we know that $v_{J^{c} J}^{\lambda}$ is continuous on $\bar{D}$. Moreover, $v_{I J *}^{\lambda} \geq v_{J^{c} J *}^{\lambda}=v_{J^{c} J}^{\lambda}$ since $v^{\lambda}$ is nondecreasing with respect to its $p$-variable. In view of Theorem 3.1 and Proposition 4.6, it thus suffices to show that $v_{I J *}^{\lambda} \geq v_{I J}^{\lambda *}$ on $\partial D_{I J} \cap[0, T) \times(0, \infty)^{d} \times[0,1]^{\kappa}$.

By Proposition 4.2 and our induction assumption, we have

$$
v_{I J}^{\lambda *} \leq v_{I^{\prime} J^{\prime}}^{\lambda *}=v_{I^{\prime} J^{\prime}}^{\lambda} \text { on } D_{I^{\prime} J^{\prime}},
$$

for all $(I, J),\left(I^{\prime}, J^{\prime}\right) \in \mathcal{P}_{\kappa}$ such that $\left(I^{\prime}, J^{\prime}\right) \supsetneq(I, J)$. Hence, it suffices to show that $v_{I J *}^{\lambda} \geq v_{I^{\prime} J^{\prime}}^{\lambda}$ on $D_{I^{\prime} J^{\prime}}$.

We now fix $\left(I^{\prime}, J^{\prime}\right) \in \mathcal{P}_{\kappa}$ such that $\left(I^{\prime}, J^{\prime}\right) \supsetneq(I, J)$. Since $v_{I J^{\prime}}^{\lambda} \geq v_{I^{\prime} J^{\prime}}^{\lambda}$, it suffices to restrict to the case $I=I^{\prime}$. By Proposition 4.4, $v_{I J *}^{\lambda}$ is a viscosity supersolution of

$$
\min \left\{\varphi-\ell,-\partial_{t} \tilde{\varphi}+F_{I J^{\prime}}^{*} \varphi\right\} \geq 0 \text { on } D_{I J^{\prime}} .
$$

On the other hand, Step 1 and Theorem 3.1 imply that $v_{I J^{\prime}}^{\lambda}$ is continuous on $D_{I J^{\prime}}$ and is a viscosity subsolution of

$$
\min \left\{\varphi-\ell,-\partial_{t} \tilde{\varphi}+F_{I J^{\prime} *} \varphi\right\} \leq 0 \text { on } D_{I J^{\prime}} .
$$

(i) First assume that $I \cup J^{\prime}=\mathcal{K}$. Then, Theorem 3.1 and Proposition 4.6 imply that $v_{I J *}^{\lambda} \geq v_{I J^{\prime}}^{\lambda}$ on $\bar{D}_{I J^{\prime}}$.

(ii) We now assume that $v_{I J *}^{\lambda} \geq v_{I J^{\prime}}^{\lambda}$ on $D_{I J^{\prime}}$ if $|I|+\left|J^{\prime}\right|=n \in(\kappa-k, \kappa]$ and show that this implies that the result also holds for $|I|+\left|J^{\prime}\right|=n-1$. Our recursion assumption implies that $v_{I J *}^{\lambda} \geq v_{I J^{\prime \prime}}^{\lambda}$ on $D_{I J^{\prime \prime}}$ for all $J^{\prime \prime} \supset J^{\prime}, J^{\prime \prime} \neq J^{\prime}$. Since $v_{I J^{\prime}}^{\lambda} \leq v_{I J^{\prime \prime}}^{\lambda}$, we have $v_{I J *}^{\lambda} \geq v_{I J^{\prime}}^{\lambda}$ on $\partial D_{I J^{\prime}}$. Moreover, (i) above 
together with the fact that $I \subset J^{\prime c}$, since $|I|+\left|J^{\prime}\right| \leq \kappa$, imply that $v_{I J *}^{\lambda} \geq v_{J^{\prime} c}^{\lambda}=v_{J^{\prime} c J^{\prime}}^{\lambda}$ on $D_{I J^{\prime}}$, On the other hand, by Theorem 3.1 and our induction assumption, $v_{I J^{\prime}}^{\lambda}$ is continuous and is a subsolution on $D_{I J^{\prime}}$ of

$$
\max \left\{\min \left\{\varphi-\ell,-\partial_{t} \varphi+F_{I J^{\prime} *}\left(\cdot, D \varphi, D^{2} \varphi\right)\right\}, M_{\lambda}^{I J^{\prime}}\left(\varphi, v_{J^{\prime} c J^{\prime}}^{\lambda}, D_{p} \varphi\right)\right\}=0
$$

while, by Proposition 4.4, $v_{I J *}^{\lambda}$ is a supersolution on $D_{I J^{\prime}}$ of

$$
\max \left\{\min \left\{\varphi-\ell,-\partial_{t} \varphi+F_{I J^{\prime}}^{*}\left(\cdot, D \varphi, D^{2} \varphi\right)\right\}, M_{\lambda}^{I J^{\prime}}\left(\varphi, v_{J^{\prime} c J^{\prime}}^{\lambda}, D_{p} \varphi\right)\right\}=0 .
$$

The fact that $v_{I J *}^{\lambda} \geq v_{I J^{\prime}}^{\lambda}$ is then a consequence of Proposition 4.6.

Proof of Theorem 3.2. We only prove item (i) of the theorem, the second one being proved similarly. We argue by induction as in the above proof.

Step 1. The fact that $V_{I J} \geq v_{I J}^{\lambda}$ on $\bar{D}_{I J}$ when $(I, J) \in \mathcal{P}_{\kappa}^{\kappa}$ is an immediate consequence of Theorem 3.1 and Proposition 4.6.

Step 2. We now assume that $V_{I J} \geq v_{I J}^{\lambda}$ on $\bar{D}_{I J}$ if $(I, J) \in \mathcal{P}_{\kappa}^{\kappa-k}$ for some $1 \leq k<\kappa$, and show that this implies that it holds for $(I, J) \in \mathcal{P}_{\kappa}^{\kappa-k-1}$.

By Step 1 and the fact that $V$ is non-decreasing with respect to its $p$-parameter, we know that $V_{I J} \geq$ $V_{J^{c} J} \geq v_{J^{c} J}^{\lambda}$ which is upper-semicontinuous by Proposition 4.7. Moreover, we have by assumption that $V_{I J *} \geq V_{I^{\prime} J^{\prime} *}$ on $\partial D_{I J} \cap D_{I^{\prime} J^{\prime}}$ for $\left(I^{\prime}, J^{\prime}\right) \in \mathcal{P}_{\kappa}$ such that $I^{\prime} \supset I$ and $J^{\prime} \supset J$ with $\left(I^{\prime}, J^{\prime}\right) \neq(I, J)$, and that $V_{I J *}(T, \cdot) \geq G^{\lambda}$. Since $v^{\lambda}$ is continuous by Proposition 4.7, our induction assumption then leads to $V_{I J *} \geq v_{I J}^{\lambda}$ on $\partial D_{I J}$. The fact that $V_{I J} \geq v_{I J}^{\lambda}$ on $\bar{D}_{I J}$ is then a consequence of Theorem 3.1 and Proposition 4.6.

\subsection{Proof of Theorem 3.4}

We first prove the convergence for $(I, J) \in \mathcal{P}_{\kappa}^{\kappa}$.

Proposition 4.8 Fix $(I, J) \in \mathcal{P}_{\kappa}^{\kappa}$. Then, $\bar{w}_{I J}^{*} \leq v_{I J}^{\lambda} \leq \bar{w}_{I J *}^{\bar{a}}$ on $\bar{D}_{I J}$. In particular,

$$
\bar{w}_{I J}^{*}=v_{I J}^{\lambda}=\bar{w}_{I J *} \text { on } \bar{D}_{I J} .
$$

Proof. 1. Recall that $\bar{w}_{I J}^{*}$ is well-defined and takes values in $[\ell, L]$. Moreover, the numerical scheme defined above is monotone and consistent under (3.19), recall in particular (3.18). Arguing as in [2], it follows that $\bar{w}_{I J}^{*}$ is a viscosity subsolution on $\bar{D}_{I J}$ of

$$
\begin{aligned}
& \max \left\{\varphi-L, H_{I J *}^{\lambda}[\varphi, 0]\right\}=0 \quad \text { on } \quad D_{I J} \\
& \min \left\{\max \left\{\varphi-L, H_{I J *}^{\lambda}[\varphi, 0]\right\}, \varphi-G^{\lambda}\right\}=0 \quad \text { on } \quad\{T\} \times(0, \infty)^{d} \times \bar{B}_{I J} .
\end{aligned}
$$

Appealing to Lemma 4.2, the above operator can be reduced to

$$
\begin{aligned}
& \max \left\{\varphi-L, H_{I J *}^{\lambda}[\varphi, 0]\right\}=0 \quad \text { on } \quad D_{I J} \\
& \varphi-G^{\lambda}=0 \quad \text { on } \quad\{T\} \times(0, \infty)^{d} \times \bar{B}_{I J} .
\end{aligned}
$$

The fact that $\bar{w}_{I J}^{*} \leq v_{I J}^{\lambda}$ then follows from Theorem 3.3 and Remark 4.1.

2. By the same arguments and Lemma 4.2 again, we deduce that $\bar{w}_{I J *}^{\bar{a}}$ is a bounded viscosity supersolution on $\bar{D}_{I J}$ of

$$
\begin{aligned}
& \max \left\{\varphi-L, H_{I J}^{\bar{a} *}[\varphi, 0]\right\}=0 \quad \text { on } \quad D_{I J} \\
& \varphi-G^{\lambda}=0 \quad \text { on } \quad\{T\} \times(0, \infty)^{d} \times \bar{B}_{I J} .
\end{aligned}
$$


Since $(I, J) \in \mathcal{P}_{\kappa}^{\kappa}, A_{I J}=\{0\}$ so that $w_{I J *}^{\bar{a}}$ and $H_{I J}^{\bar{a} *}$ do not depend on $\bar{a}$. The fact that $v_{I J}^{\lambda} \leq \bar{w}_{I J *}^{\bar{a}}=$ $\bar{w}_{I J *}$ on $\bar{D}_{I J}$ then follows from Remark 4.1 and the fact that $v_{I J}^{\lambda}$ is a subsolution of the latter, by Theorem 3.3.

We now complete the proof by an induction argument.

Proposition 4.9 Fix $(I, J) \in \mathcal{P}_{\kappa} \backslash \mathcal{P}_{\kappa}^{\kappa}$. Then, $\bar{w}_{I J}^{*} \leq v_{I J}^{\lambda} \leq \bar{w}_{I J *}^{\bar{a}}$ on $\bar{D}_{I J}$. In particular, $\bar{w}_{I J}^{*}=v_{I J}^{\lambda}=$ $\bar{w}_{I J *}$ on $\bar{D}_{I J}$.

Proof. In view of Proposition 4.8, we can argue by induction. We therefore assume that

$$
\bar{w}_{I J *}^{\bar{a}} \geq v_{I J}^{\lambda} \geq \bar{w}_{I J}^{*} \text { on } \bar{D}_{I J} \text { for all }(I, J) \in \mathcal{P}_{\kappa}^{k}
$$

for some $1 \leq k \leq \kappa$, and show that this implies that it holds for $(I, J) \in \mathcal{P}_{\kappa}^{k-1}$ as well.

1. By the same argument as in Proposition 4.8, Lemma 4.3, and (4.57), we first obtain that $\bar{w}_{I J}^{*}$ is a bounded viscosity subsolution on $\bar{D}_{I J}$ of

$$
\left\{\begin{array}{c}
\left(\varphi-v_{J^{c} J}^{\lambda}\right) \wedge \max \left\{\varphi-L, H_{I J *}^{\lambda}\left[\varphi, v_{J^{c} J}^{\lambda}\right]\right\}=0 \text { on } D_{I J} \\
\varphi-v_{I^{\prime} J^{\prime}}^{\lambda}=0 \text { on } D_{I^{\prime} J^{\prime}} \cup\left(\{T\} \times(0, \infty)^{d} \times B_{I^{\prime} J^{\prime}}\right),\left(I^{\prime}, J^{\prime}\right) \supsetneq(I, J) \\
\varphi-G^{\lambda}=0 \text { on }\{T\} \times(0, \infty)^{d} \times B_{I J} .
\end{array}\right.
$$

On the other hand, Theorem 3.3 implies that $v_{I J}^{\lambda}$ is a supersolution of (4.59) on $\bar{D}_{I J}$ with $H_{I J}^{\lambda *}$ in place of $H_{I J *}^{\lambda}$, that $v_{I J}^{\lambda}=v_{I^{\prime} J^{\prime}}^{\lambda}$ on $\partial D_{I J} \cap \bar{D}_{I^{\prime} J^{\prime}}$ and $v_{I J}^{\lambda}(T, \cdot)=G^{\lambda}(T, \cdot)$ on $(0, \infty)^{d} \times \bar{B}_{I J}$. Finally, $v_{I J}^{\lambda} \geq v_{J^{c} J}^{\lambda}$ since it is non-decreasing in its $p$-parameter. The fact that $\bar{w}_{I J}^{*} \leq v_{I J}^{\lambda}$ on $\bar{D}_{I J}$ then follows from Remark 4.1 .

2. By the same reasoning, recall in particular Lemma 4.3 and the first assertion of Proposition 4.8, $\bar{w}_{I J *}^{\bar{a}}$ is a bounded viscosity supersolution on $\bar{D}_{I J}$ of

$$
\left\{\begin{array}{c}
\left(\varphi-v_{J^{c} J}^{\lambda}\right) \wedge \max \left\{\varphi-L, H_{I J}^{\bar{a} *}\left[\varphi, v_{J^{c} J}^{\lambda}\right]\right\}=0 \text { on } D_{I J} \\
\max \left\{\left(\varphi-v_{J^{c} J}^{\lambda}\right) \wedge \max \left\{\varphi-L, H_{I J^{\prime}}^{\bar{a} *}\left[\varphi, v_{J^{\prime c} J^{\prime}}^{\lambda}\right]\right\}, \varphi-v_{I^{\prime} J^{\prime}}^{\lambda}\right\}=0 \text { on } D_{I^{\prime} J^{\prime}},\left(I^{\prime}, J^{\prime}\right) \supsetneq(I, J) \\
\varphi-G^{\lambda}=0 \text { on }\{T\} \times(0, \infty)^{d} \times \bar{B}_{I J}
\end{array}\right.
$$

where $v_{J^{c} J}^{\lambda}=\bar{w}_{J^{c} J}^{\bar{a} *}=\bar{w}_{J^{c} J *}^{\bar{a}}$ and $v_{J^{\prime} c J^{\prime}}^{\lambda}=\bar{w}_{J^{\prime c} J^{\prime}}^{\bar{a}^{*}}=\bar{w}_{J^{\prime c} J^{\prime} *}^{\bar{a}_{I J}}$ are continuous.

On the other hand, $v_{I J}^{\lambda}$ is a subsolution of $\max \left\{\varphi-L, H_{I J *}^{\lambda}\left[\varphi, v_{J^{c} J}^{\lambda}\right]\right\}=0$ on $\bar{D}_{I J}$ and satisfies the boundary condition $v_{I J}^{\lambda}=v_{I^{\prime} J^{\prime}}^{\lambda}$ on $\partial D_{I J} \cap D_{I^{\prime} J^{\prime}}$ and $v_{I J}^{\lambda}=G^{\lambda}$ on $\{T\} \times(0, \infty)^{d} \times \bar{B}_{I J}$, recall Theorem 3.3. In view of Remark 4.1, and the fact that $\bar{w}_{I J *}^{\bar{a}} \geq v_{J^{c} J}^{\lambda}$ on $D_{I J}$ and $\bar{w}_{I J *}^{\bar{a}} \geq G^{\lambda}$ on $\{T\} \times(0, \infty)^{d} \times \bar{B}_{I J}$, by its supersolution property, it only remains to prove that $\bar{w}_{I J *}^{\bar{a}} \geq v_{I^{\prime} J^{\prime}}^{\lambda}$ on $\partial D_{I J} \cap D_{I^{\prime} J^{\prime}}$.

Since $v_{I J^{\prime}}^{\lambda} \geq v_{I^{\prime} J^{\prime}}^{\lambda}$, it suffices to consider the case $I=I^{\prime}$. If $\left(I, J^{\prime}\right) \in \mathcal{P}_{\kappa}^{\kappa}$, then the result follows from Remark 4.1, Theorem 3.3 and the second and third equations in (4.60). Assuming that $\bar{w}_{I J *}^{\bar{a}} \geq v_{I J^{\prime}}^{\lambda}$ on $\partial D_{I J} \cap D_{I J^{\prime}}$ for $\left(I, J^{\prime}\right) \in \mathcal{P}_{\kappa}^{k}$ with $|I|+|J|+2 \leq k \leq \kappa$, then we deduce similarly that it holds for $\left(I, J^{\prime}\right) \in \mathcal{P}_{\kappa}^{k-1}$, since our induction assumption guarantees the required boundary conditions.

\section{References}

[1] G. Barles (1994). Solutions de Viscosité des Equations d'Hamilton-Jacobi, Springer-Verlag, Berlin.

[2] G. Barles and P. E. Souganidis (1991). Convergence of approximation schemes for fully nonlinear second order equation. Asymptotic Analysis, 4, 271-283.

[3] I. Bentahar and B. Bouchard (2006). Barrier option hedging under constraints: A viscosity approach, SIAM Journal on Control and Optimization, 41, 404-424. 
[4] B. Bouchard (2002). Stochastic Target with Mixed diffusion processes, Stochastic Processes and their Applications, 101, 273-302.

[5] B. Bouchard and N. M. Dang (2010). Generalized stochastic target problems for pricing and partial hedging under loss constraints - Application in optimal book liquidation, preprint.

[6] B. Bouchard, R. Elie and N. Touzi (2009). Stostastic Target problems with controlled loss, SIAM Journal on Control and Optimization, 48 (5), 3123-3150.

[7] F. Camilli and M. Falcone (1995), An approximation scheme for the optimal control of diffusion processes, Mathematical Modelling and Numerical Analysis, 29, 1, 97-122.

[8] M.G. Crandall, H. Ishii and P.-L Lions (1992). User's guide to viscosity solutions of second order partial differential equations, Amer. Math. Soc., 27, 1-67.

[9] J. Cvitanic, H. Pham and N. Touzi (1999). Super-replication in stochastic volatility models with portfolio constraints, Journal of Applied Probability 36, 523-545.

[10] H. Föllmer and D. Kramkov (1997). Optional decomposition under constraints, Probability Theory and Related Fields, 109, 1-25.

[11] H. Föllmer and P. Leukert (1999). Quantile hedging, Finance and Stochastics, 3, 251-273.

[12] D. W. Moore (1993). Simplical mesh generation with applications. Phd Thesis, Report no 92-1322, Cornell University.

[13] R.T. Rockafellar (1970). Convex Analysis. Princeton University Press, Princeton, NJ.

[14] H.M. Soner and N. Touzi (2002). Dyanmic programming for stochastic target problems and geometric flows, Journal of European Mathematical Society, 4, 201-236.

[15] H.M. Soner and N. Touzi (2002). Stochastic target problems, dynamic programming and visosity solutions, SIAM Journal on Control and Optimization, 41, 404-424.

[16] H.M. Soner and N. Touzi (2004). The problem of super-replication under constraints, ParisPrinceton Lectures in Mathematical Finance, Lecture Notes in Mathematics, Springer-Verlag. 